Florida International University

FIU Digital Commons

FIU Electronic Theses and Dissertations

University Graduate School

$11-13-2015$

\title{
Development of a Two-Fluid Drag Law for Clustered Particles Using Direct Numerical Simulation and Validation through Experiments
}

Ahmadreza Abbasi Baharanchi

Florida International University, aabba003@fiu.edu

DOI: $10.25148 /$ etd.FIDC000235

Follow this and additional works at: https://digitalcommons.fiu.edu/etd

Part of the Acoustics, Dynamics, and Controls Commons, Applied Mechanics Commons, Computer-Aided Engineering and Design Commons, Engineering Mechanics Commons, Materials

Chemistry Commons, Numerical Analysis and Computation Commons, Ordinary Differential Equations and Applied Dynamics Commons, Other Mechanical Engineering Commons, Partial Differential Equations Commons, and the Physical Chemistry Commons

\section{Recommended Citation}

Abbasi Baharanchi, Ahmadreza, "Development of a Two-Fluid Drag Law for Clustered Particles Using Direct Numerical Simulation and Validation through Experiments" (2015). FIU Electronic Theses and Dissertations. 2489.

https://digitalcommons.fiu.edu/etd/2489 


\section{FLORIDA INTERNATIONAL UNIVERSITY}

Miami, Florida

DEVELOPMENT OF A TWO-FLUID DRAG LAW FOR CLUSTERED

PARTICLES USING DIRECT NUMERICAL SIMULATION AND VALIDATION

THROUGH EXPERIMENTS

A dissertation submitted in partial fulfillment of

the requirements for the degree of

DOCTOR OF PHILOSOPHY

in

MECHANICAL ENGINEERING

by

Ahmadreza Abbasi Baharanchi

2016 
To: Interim Dean Ranu Jung

College of Engineering and Computing

This dissertation, written by Ahmadreza Abbasi Baharanchi, and entitled Development of a Two-fluid Drag Law for Clustered Particles using Direct Numerical Simulation and Validation through Experiments, having been approved in respect to style and intellectual content, is referred to you for judgment.

We have read this dissertation and recommend that it be approved.

Norman Munroe

Cheng-Xian Lin

Bilal El-Zahab

Leonel Lagos

Shankar Subramaniam

George S. Dulikravich, Major Professor

Date of Defense: November 13, 2015

The dissertation of Ahmadreza Abbasi Baharanchi is approved.

Interim Dean Ranu Jung College of Engineering and Computing

Andrés G. Gil

Vice President for Research and Economic

Development and Dean of the University Graduate School

Florida International University, 2016 
CCopyright 2016 by Ahmadreza Abbasi Baharanchi

All rights reserved. 


\section{DEDICATION}

I dedicate this dissertation to my wonderful and pretty wife, to my parents and family as they have always encouraged me to follow my dreams. I am excited to share with them what comes next. 


\section{ACKNOWLEDGMENTS}

I would first like to recognize Dr. George S. Dulikravich and Dr. Norman Munroe for their supervision of the present work and their wonderful help and support. To the other members of my committee, Dr. Cheng-Xian Lin, Dr. Bilal Elzahab, Dr. Leonel Lagos, and Dr. Shankar Subramaniam, thank you for the feedback, direction, and assistance when I needed it.

Special thanks are given to Dr. Seckin Gokaltun as the PI of the funded project related to this dissertation for excellent mentorship and coordination of financial support until completion of this dissertation. I would like to extend my sincerest regards to collaborators of the project from the Iowa State University, Dr. Subramaniam and Mr. Mehrabadi, for their incredible inputs and feedbacks.

Finally, and most importantly, I would like to thank my family, and in special my wife, for their wonderful support, encouragement, patience and love over the past five years. Their companionship in my happiness, hardship and sorrow reflected their magnificent devotion and love. I thank my parents, for their faith in me and for supporting me to follow my ambitions far away from home and around the world.

My dissertation research was made possible thanks to funding and support from the Department of Energy's National Energy Technology Laboratory under the grant number DEFE0007260, the Department of Mechanical Engineering, the Applied Research Center division, MAIDROC laboratory, WOW laboratory, AMERI laboratory, High Performance Computing division, and the University Graduate School at Florida International University. 
ABSTRACT OF THE DISSERTATION

DEVELOPMENT OF A TWO-FLUID DRAG LAW FOR CLUSTERED PARTICLES

USING DIRECT NUMERICAL SIMULATION AND VALIDATION THROUGH

EXPERIMENTS

by

\author{
Ahmadreza Abbasi Baharanchi \\ Florida International University, 2016 \\ Miami, Florida \\ Professor George S. Dulikravich, Major Professor
}

This dissertation focused on development and utilization of numerical and experimental approaches to improve the CFD modeling of fluidization flow of cohesive micron size particles. The specific objectives of this research were: (1) Developing a cluster prediction mechanism applicable to Two-Fluid Modeling (TFM) of gas-solid systems (2) Developing more accurate drag models for Two-Fluid Modeling (TFM) of gas-solid fluidization flow with the presence of cohesive interparticle forces (3) using the developed model to explore the improvement of accuracy of TFM in simulation of fluidization flow of cohesive powders (4) Understanding the causes and influential factor which led to improvements and quantification of improvements (5) Gathering data from a fast fluidization flow and use these data for benchmark validations. Simulation results with two developed cluster-aware drag models showed that cluster prediction could effectively influence the results in both the first and second cluster-aware models. It was proven that improvement of accuracy of TFM modeling using three versions of the first 
hybrid model was significant and the best improvements were obtained by using the smallest values of the switch parameter which led to capturing the smallest chances of cluster prediction. In the case of the second hybrid model, dependence of critical model parameter on only Reynolds number led to the fact that improvement of accuracy was significant only in dense section of the fluidized bed. This finding may suggest that a more sophisticated particle resolved DNS model, which can span wide range of solid volume fraction, can be used in the formulation of the cluster-aware drag model. The results of experiment suing high speed imaging indicated the presence of particle clusters in the fluidization flow of FCC inside the riser of FIU-CFB facility. In addition, pressure data was successfully captured along the fluidization column of the facility and used as benchmark validation data for the second hybrid model developed in the present dissertation. It was shown the second hybrid model could predict the pressure data in the dense section of the fluidization column with better accuracy. 


\section{TABLE OF CONTENTS}

CHAPTER

PAGE

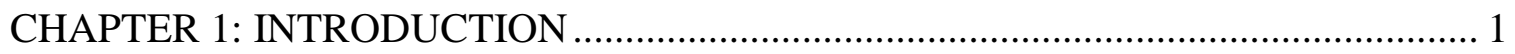

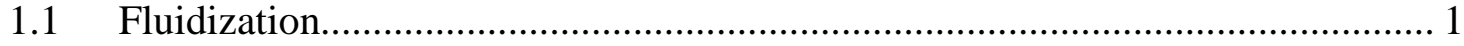

1.2 Numerical Simulation of Fluidization Flows .................................................. 6

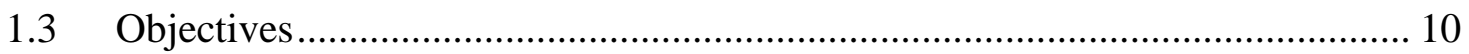

1.4 Dissertation Organization............................................................................ 11

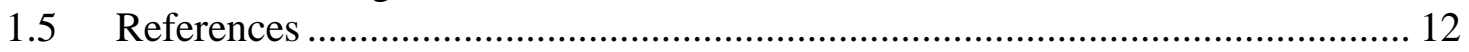

CHAPTER 2: IMPROVEMENT OF EXISTING STANDARD TWO-FLUID DRAG MODELS IN SIMULATION OF GAS-SOLID FLOWS USING A PR-DNS DRAG

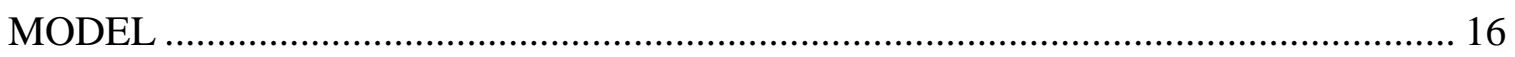

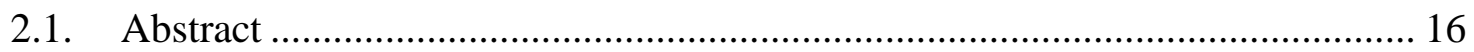

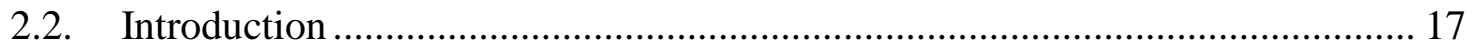

2.3. Governing Equations...................................................................................... 21

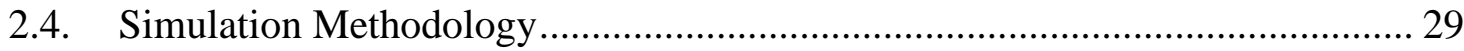

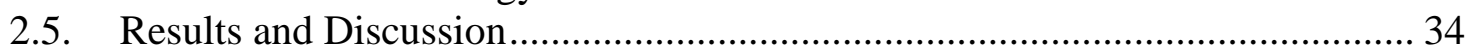

2.5.1. Simulation Results with Relaxed Constraints ................................................... 39

2.5.2. Switching Frequency and Improvement in Hybrid Model................................. 47

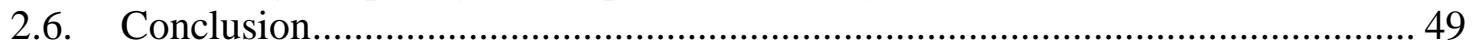

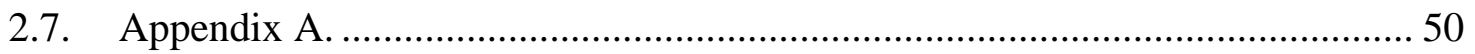

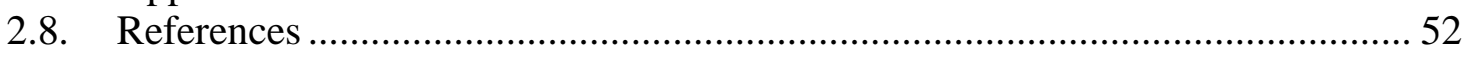

CHAPTER 3: INVESTIGATION OF PARAMETERS CONTRIBUTING TO IMPROVEMENTS OF TWO FLUID MODELING OF A FLUDIZATION FLOW...... 55

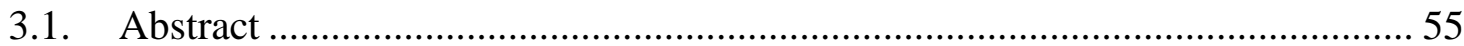

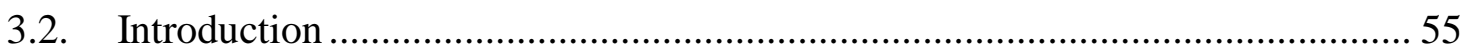

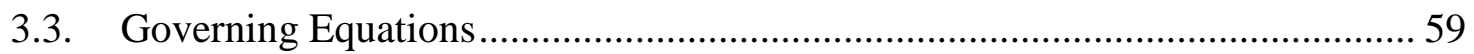

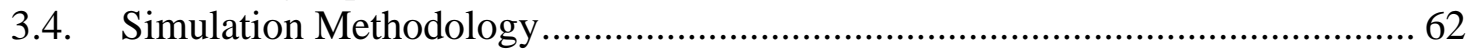

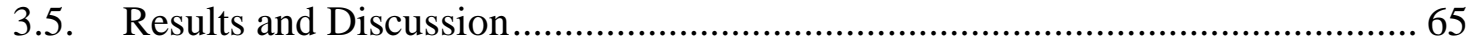

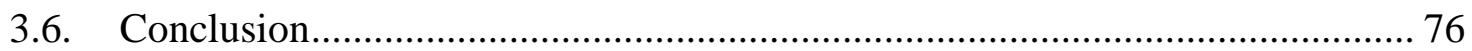

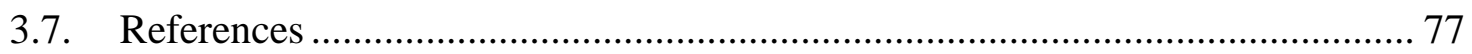

CHAPTER 4: A CLUSTER-AWARE DRAG MODEL FOR SIMULATION OF

PARTICLE-GAS FLOWS INVOLVING COHESIVE INTERPARTICLE FORCES ... 81

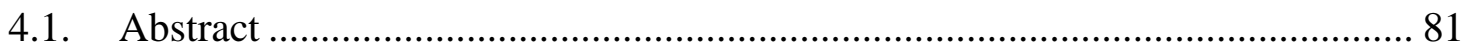

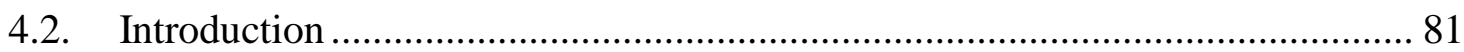

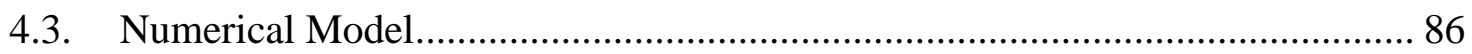

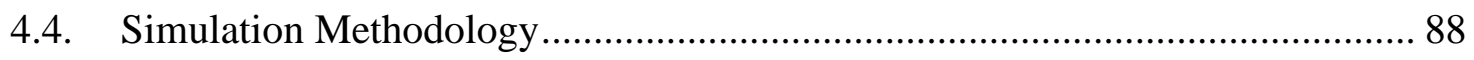

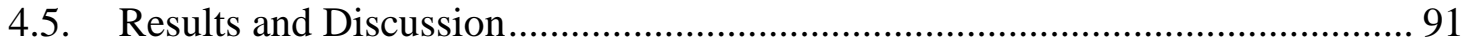

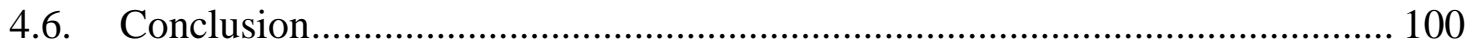

4.7. References .............................................................................................. 101

CHAPTER 5: SIMULATION OF FAST FLUIDIZATION FLOW INSIDE THE FIU_CFB FACILITY USING ENHANCED CLUSTER-AWARE DRAG MODELS

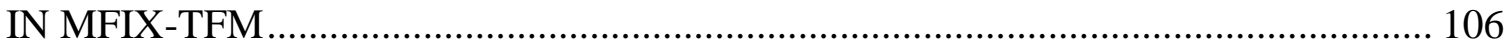

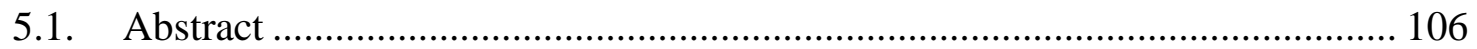




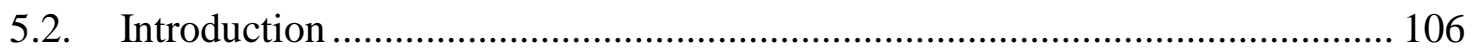

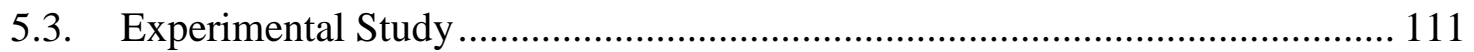

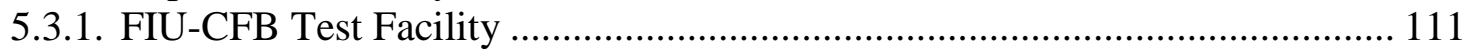

5.3.2. Air Flow Rate Measurement ......................................................................... 112

5.3.3. Solid Density and Flow Rate Measurement ........................................................ 114

5.3.4. Performance Checkup Test of Data Acquisition Unit...................................... 118

5.3.5. Performance Checkup Test of Pressure Transducers......................................... 119

5.3.6. Particle Size Distribution Analysis ............................................................... 121

5.3.7. Pressure Measurement in the Fast Fluidization Experiment............................. 122

5.3.8. Cluster Detection Inside the Fluidization Column Using High Speed Camera126

5.4. Simulation of Fluidization Flow inside FIU-CFB Facility .............................. 127

5.4.1. Simulation Methodology......................................................................... 127

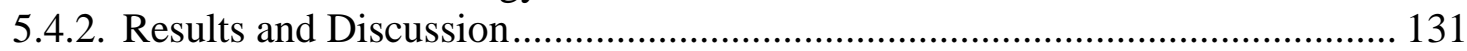

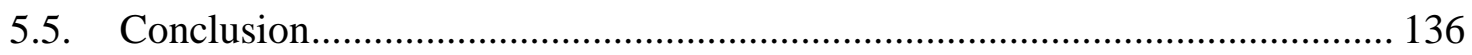

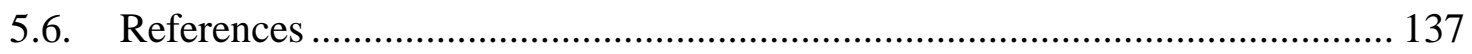

CHAPTER 6: SUMMARY AND CONCLUSION ………......................................... 141

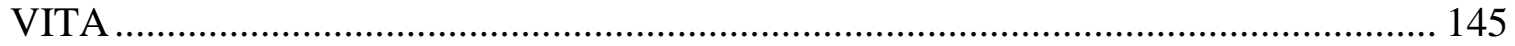




\section{LIST OF TABLES}

TABLE

PAGE

Table 2-1 Proposed drag model scheme using the cohesive index for MFIX-TFM simulations

Table 2-2 Governing equations for the existing drag models used for switching procedure

Table 2-3 Drag model combinations tested in this study

Table 2-4 Set up parameters for MFIX_TFM simulations

Table 2-5 Thresholds for solid volume fraction and granular temperature to prevent switching from the standard model to the TGS model

Table 2-6 Error in numerical simulation compared to available experimental data of Li and Kwauk (1998) for computational grid size of $20 \times 150$

Table 2-7 Variation of the thresholds in the AGDSM models

Table 2-8 Error calculations and best improvements for different versions of the AGDSM model

Table 3-1 Governing Equation for the TFM

Table 3-2 Table 3-2 Governing equations for the existing drag models used for switching procedure

Table 4-1 Proposed hybrid drag model scheme using the cohesive index for MFIX-TFM simulations

Table 4-2 Error calculations and best improvements for the AGDSM11 model

Table 5-1 Mass flow rate and Mass flux calculated for the FCC in different valve openings

Table 5-2 Test conditions for different air flows and FCC mass fluxes

Table 5-3 Parameters for MFIX_TFM simulations of FIU-CFB case 8 (570-22) 


\section{LIST OF FIGURES}

FIGURE

PAGE

Figure 1-1 Flow regime map of gas/solid contacting (Kunii \& Levenspiel, 1991)

Figure 1-2 Powder classification diagram for fluidization (Cocco et al., 2014)

Figure 2-1 Computational domain and boundary conditions used for MFIX-TFM fluidized bed flow simulations, (a) $20 \times 150$, (b) 40 $\times 300$, (c) $60 \times 450$

Figure 2-2 Profile of solid volume fraction along the riser of the fluidized bed, MFIX-TFM results with TGS and existing drag models on three computational grid sizes

Figure 2-3 Switching effects in the three versions of the AGDSM model on time and area-averaged profile of the solid volume fraction along the riser against variation of the Ha-THS

Figure 2-4 Profile of solid volume fraction $\left(\varepsilon_{s}\right)$ from MFIX_TFM simulations for various values of Ha-THS; (a to c) represent AGDSM1, ( $\mathrm{d}$ to $\mathrm{f}$ ) represent the AGDSM2, and (g to i) represent the AGDSM3

Figure 2-5 Error in TFM simulation for three versions of the proposed drag model, maximum of error on left and averaged relative error on right.

Figure 2-6 Optimal MFIX-TFM simulations by versions of the AGDSM model, (a) to (c) are versions of the AGDSM model versus corresponding standard models, (d): best result from AGDSM13 versions and the profile of Hong et al. (2012)

Figure 2-7 Fourth-order fit to the experimental data of Li and Kwauk (1994)

Figure 2-8 Frequency of switch operations. (a) constituent drag models, (b) proposed AGDSM model versions in optimal cases

Figure 3-1 Optimal MFIX-TFM simulations by versions of the AGDSM model, (a) to (c) are versions of the AGDSM model versus corresponding standard models, (d): best result from AGDSM13 versions and the profile of Hong et al. (2012) 
Figure 3-2 Contour of solid volume fraction averaged over the last 1000 time steps

Figure 3-3 Frequency of switch operations. (a) constituent drag models, (b) proposed AGDSM model versions in optimal cases

Figure 3-4 Modification due to cluster formation (cells meeting the criteria of $\mathrm{Ha}>\mathrm{Ha}-\mathrm{THS}$ for switch operations)

Figure 3-5 Contour of drag force averaged over the last 1000 time steps

Figure 3-6 Averaged values of drag forces (a-b) and normalized drag forces (c-d)

Figure 3-7 Comparisons of spatially-averaged variables over the 100 last time steps: (a) drag force (b) normalized drag force

Figure 4-1 Computational domain and boundary conditions used for MFIX-TFM fluidized bed flow simulations

Figure 4-2 Profile of solid volume fraction along the riser obtained by MFIX_TFM simulations for different values of Ha-THS, experimental data by Li and Kwauk (1994)

Figure 4-3 Error in TFM simulation for three versions of the proposed drag model, maximum of error on left and averaged relative error on right

Figure 4-4 Comparison of performance of hybrid models, Cluster-aware drag model proposed in this work in dashed line (Ha-THS = $1 \times 10-4)$ and empty circles (Ha-THS $=1 \times 10-10)$ and AGDSM1 from Abbasi et al. (2015) for Ha-THS $=1 \times 10-10$

Figure 5-1 Circulation fluidization bed (CFB) for fluidization test at FIU

Figure 5-2 LabView ${ }^{\circledR}$ VI designed for communication with pressure transducer and thermocouple on the Field point 2010 for the purpose of data reception and storage.

Figure 5-3 Solid mass flow rate measurement experiment (on left), and solid sliding valve at the fully closed position $\mathrm{d}=1$ ")

Figure 5-4 Mass flow rate measurement for FCC in different valve openings

Figure 5-5 Schematic of the first (on left) and second (on right) data 118 performance checkup test for the data acquisition module with 
Filed point 2010 control module

Figure 5-6 Sample performance check of pressure transducers (a) using increment of 0.01psi for excitation pressures controlled by manometer (b) comparison of different methods and factory data

Figure 5-7 Shaker machine, implementing sieving mechanism to categorize particles based on size

Figure 5-8 Pressure data for the case 540-22, Vair $=1.24 \mathrm{~m} / \mathrm{s}$ and $\mathrm{mFCC}=$ $40(\mathrm{~g} / \mathrm{s})$

Figure 5-9 Time-average pressure profile along the fluidization column of FIU-CFB for different air flow and solid mass flow rates (a) blower speed $=540 \mathrm{rpm}$ (b) blower speed $=570 \mathrm{rpm}$

Figure 5-10 Cluster visualization in fast fluidization experiment (FIU-CFB). (a) Imaging set up, (b) Image with statics on the riser wall (c) noise-free image and portray of few clusters

Figure 5-11 Computational domain representing the fluidization problem, (on left): schematic of the computational domain with boundary values, (on right): computational grid $(30 \times 150)$

Figure 5-12 Contour of void fraction ( $8 \mathrm{~g}$ ) from three TFM simulations of the fast fluidization flow inside FIU-CFB, drag models: Fdrag.std (Syam-O’Brien), AGDSM1 (Fdrag.std $\rightleftharpoons$ TGS), and AGDSM11 (Fdrag.std $\rightleftharpoons \mathrm{g}(\mathrm{Re}) *$ Fdrag.std)

Figure 5-13 comparison of pressure profiles along the fluidization column of FIU-CFB 


\section{CHAPTER 1: INTRODUCTION}

\subsection{Fluidization}

Increased demand of imported petroleum, ongoing deregulation of the energy industry, and environmental concerns associated with the use of fossil fuel for production of electricity and transportation fuels are among many factors contributing to an increasing interest in better utilization of fossil fuels. Therefore, it is important to enhance our understanding of the processes involved in the conversion and utilization of fossil fuels, such as gasification, catalytic cracking, and combustion in circulating fluidized bed risers and transport reactors.

Fluidization is the process of lifting a column of solid or a bed of particles by an upwardflowing fluid (in the case of vertical fluidization flow). In gas-solid fluidization systems, the flowing gas imposes enough drag force to overcome the gravity of the solid particles. The underlying function of fluidization is to afford contact between a gas and a large inventory of solid surface per unit bed volume (Yerushalmi et al., 1975). Fluidized beds operate in variety of regimes, between which transitions happen by increase the velocity of the uplifting gas. Circulating fluidized bed (CFB) is a special type of gas- solid contactor in which fine solid particles are transported vertically in the riser by a high velocity gas stream. After exiting the top of the riser, the solids are separated from the gas stream and recirculated to the base (Berrutit and Kalogerakis, 1989). The technology of circulating fluidized beds has a wide field of application for catalytic chemical reactions, pharmaceutical production, mineral processing and combustion processes. The special 
attention of the industry in this type of fluidized bed which operates in fast fluidization regime, as explained later, is the favorability of this model in easy control of the whole process and repeated use of the solid phase.

The flow regime obtained by fluidizing the FCC particles at various superficial velocities can be estimated using the flow regime map given in Figure 1-1. This map categorizes the flow regimes based upon characteristic parameters defined as dimensionless particle size, $d_{p}^{*}\left(=d_{p}\left[\rho_{s}\left(\rho_{s}-\rho_{g}\right) g / \mu_{g}{ }^{2}\right]^{1 / 3}\right)$ and dimensionless gas velocity, $u^{*}\left(=u_{g}\left[\rho_{g} /\left(\mu_{g}\left(\rho_{s}-\right.\right.\right.\right.$ $\left.\left.\left.\rho_{\mathrm{g}}\right) \mathrm{g}\right)\right]^{1 / 3}$ (Kunii \& Levenspiel, 1991). In these expressions, d, u, $\rho, \mu, \mathrm{g}$ are the diameter, superficial gas velocity (the volumetric flow rate of the inlet gas divided by the inlet area), density, dynamic viscosity, and the acceleration of gravity, respectively and the subscripts $\mathrm{p}$ and g refer to particle and gas.

Fast fluidization regime, as of special attention of the author, that can be regarded as essentially a dense suspension marked by vigorous and intensive back mixing of solid as described by Yerushalmi et al. (1975). This regime of the flow has earned special attention in the industry for its high processing capacities due to sufficiently high carry over rates from the top of the fluidized bed column. According to Yerushalmi et al., (1976) fast fluidized bed affords excellent contact between gas and solid, can handle cohesive solids and might prove easier to scale up than a bubbling fluidized bed.

A typical example of the powder used extensively in the petrochemical industry is the Fluid Cracking Catalyst (FCC) with physical characteristics of $\overline{\mathrm{d}}_{\mathrm{p}}=50-100 \mu \mathrm{m}$ and $\rho_{\mathrm{s}} \leq$ 
$1500 \mathrm{~kg} / \mathrm{m}^{3}$, (depending on the freshness of the powder), according to Geldart (1972), Ye et al. (2005a), Wang et al. (2009), Cocco et al. (2011). The dimensionless particle diameter for FCC catalysts is found to be $\mathrm{d}_{\mathrm{p}}^{*} \approx 3$, for which minimumfluidizationvelocity intherangeof $\mathrm{u}_{\mathrm{mf}}=\left[2.86 \times 10^{-3}\right.$ to $\left.7.15 \times 10^{-3}\right] \mathrm{m} / \mathrm{s}$, minimum bubbling velocity in the range of $u_{\mathrm{mb}}=\left[5.72 \times 10^{-2}\right.$ to $\left.6.87 \times 10^{-1}\right] \mathrm{m} / \mathrm{s}$, and fast fluidization velocity in the range of $u_{\mathrm{f}}=$ [1.15 to 4.5$] \mathrm{m} / \mathrm{s}$ are obtained.

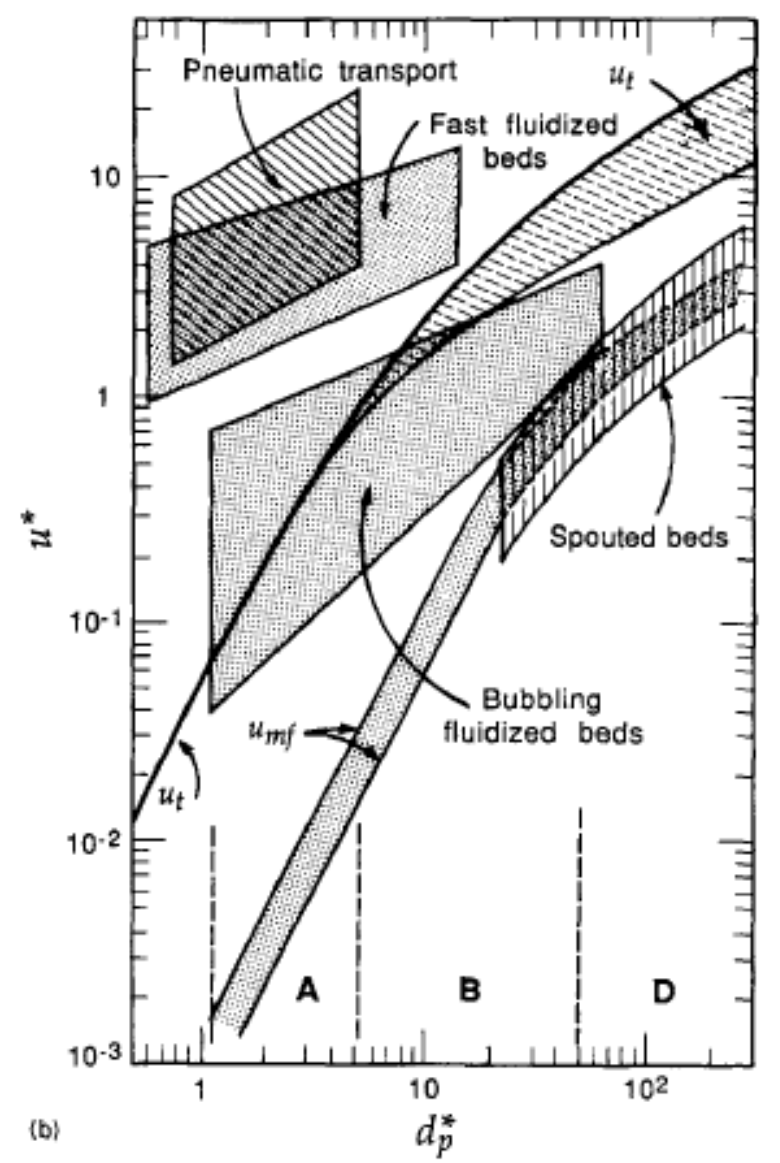

Figure 1-1 Flow regime map of gas/solid contacting (Kunii \& Levenspiel, 1991).

Type of particle can be classified based on the behavior of particles in fluidization flow. Geldart (1972) introduced A, B, C and D categories of particles based on their density 
and mean particle diameter, defined as $\overline{\mathrm{d}_{\mathrm{p}}}=1 / \sum_{\mathrm{i}=1}^{\mathrm{N}}\left(\mathrm{x}_{\mathrm{i}} / \mathrm{d}_{\mathrm{sv}_{\mathrm{i}}}\right)$. In this definition, $\mathrm{i}, \mathrm{x}$, and $\mathrm{d}_{\mathrm{sv}}$ refer to sampling number, and the mass fraction and size of the sub-group of the particles separated by sieving mechanism. The sieving procedure will be explained in the experimental section of the present dissertation. This classification is shown in Figure $1-2$.

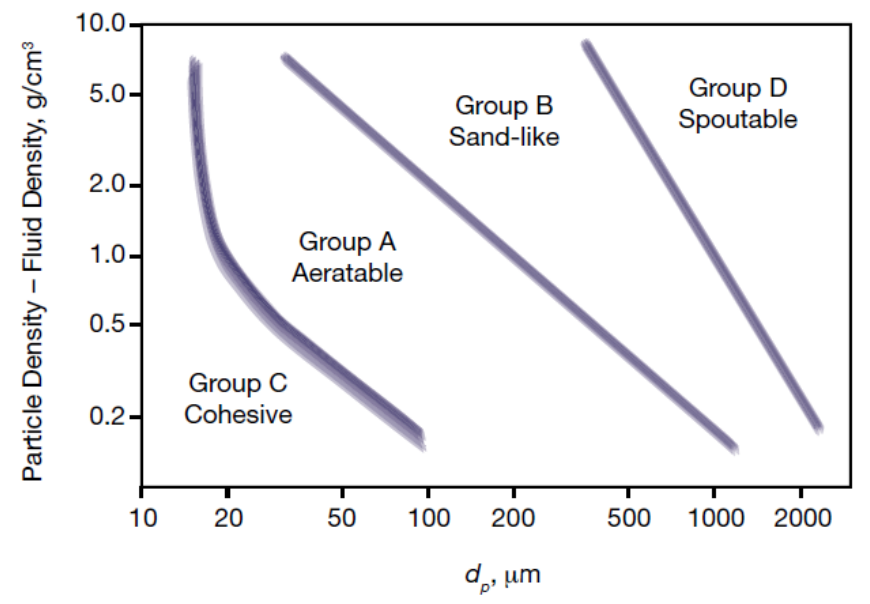

Figure 1-2 Powder classification diagram for fluidization (Cocco et al., 2014)

According to Cocco et al. (2014), Geldart A particles are defined as aeratable particles, which normally have a small particle size $(\mathrm{dp}<125 \mu \mathrm{m})$ and low particle density $(<1400$ $\mathrm{kg} / \mathrm{m} 3$ ). According to Ye et al. (2005b), this type of powder can be easily fluidized at ambient conditions and provides a homogeneous bed expansion. Particles in the group B have diameters between 150 to $1500 \mu \mathrm{m}$, do not undergo homogeneous bed expansion, and can be fluidized very easily. Coal, as an example with such characteristics, is widely used in most fluidized bed combustors and pyrolysis units with few difficulties (Cocco et al., 2014). This group tends to form large gas bubbles in gas-solid systems and slugging can occur even in columns with large diameters. Group C particles have diameters less 
than $30 \mu \mathrm{m}$ and due to their strong cohesive nature, they are the most difficult type to fluidize. This group behave more as clusters than single particles. Group D have the largest particle size and form enormous bubbles. Slugging is the staple feature of this type and this type is usually used in the spouting beds where the gas moves primarily through the center of the pipe.

The special attention to the Geldart A group of particles in this dissertation is their vastest application in the industrial fluidized beds (Cocco et al., 2014). A typical example of this group is the Fluid Cracking Catalyst (FCC), which is used in production of almost threequarter of all polyolefin in fluidized-bed processes. Fluidization flow of this powder was studied by many researchers such as Ye et al. (2005a\&b, 2008), Cocco et al. (2014), and Zimmermann and Taghipour (2005). FCC was found in the experiments of Li et al. (1991), Yang and Leu (2009), Cocco et al. (2010, 2011) and Xu and Zhu (2011) to form clusters in the fast fluidization regime of the flow.

Agglomeration of very small particles commonly named as "fines" is a typical behavior of cohesive powders used in fluidized bed columns. McKeen and Pugsley (2003) explained that cohesive interparticle forces can lead to agglomeration of Geldart A type powder and significantly affect the fluidization quality. Cocco et al. (2010), declared that cohesive forces such as electrostatics, capillary and van der Waals forces, appear to play a significant role in particle cluster formation. According to McKeen and Pugsley (2003), particle clustering can result in larger effective particle sizes and this change reduces the drag forces between the gas and solid phases. According to Cocco et al. (2010), the 
clustering of particles in circulating fluidized beds continues to be a fundamental issue in granular-fluid hydrodynamics. This effect is so strong in fluidization of group C that special mechanisms such as baffles, micro jets pulsing, and or mechanical vibrations are needed (Cocco et al., 2010). Here, the clustering issue associated with the Geldart A group is given special importance due to wide use of this particle type in the industry. FCC is used in this dissertation to represent the Geldart A group for its important and vast utilization in academic and industrial research. This powder will contribute to development and testing of drag correlations in this dissertation that can better predict the solid concentration along the fluidization column in the presence of particle clustering.

\subsection{Numerical Simulation of Fluidization Flows}

Experimental work in fluidization may involve measurement of various field data such as, air velocity (using flow meter and etc.), particle/cluster velocity and granular temperature (using high speed imaging, PIV and/or optical probes), bed expansion (digital or visual height indicators), solid concentration (using optical probe and high speed imaging). Although the most accurate and reliable method to evaluate the performance of the industrial units is direct experimentation, it is often very expensive and time consuming to manufacture and utilize test facilities. This problem is augmented in the case of parametric studies of fluidization processes for research and development and optimization purposes. For this reason, growing attention has been diverted to computational simulation of the processes by developers of gasifiers, combustors, chemical reactors, and owners of energy power plants. Indeed, computer aided design (CAD) packages have come to help us to virtually manufacture plants and assemble the 
systems, perform variety of tests using broadly varied boundary conditions, and analyze the results using optimization packages in order to recommend the optimum inputs leading to the best yield products in a matter of hours or days. In addition, as there still lacks a thorough understanding of the fluidization hydrodynamics in such applications, computational fluid dynamics (CFD) is a potential tool for troubleshooting, design, and scale-up (McKeen and Pugsley, 2003).

The downside of relying on the computer simulations is the accuracy and speed in simulation of complex processes. The simplifications to the governing equations, assumptions imposed on the ambient and boundary conditions which may not exactly represent the true problem and utilization of models which rely on empirical relations obtained from erroneous regression, interpolation or extrapolation operations have limited the accuracy of numerical modeling of many complex multiphase processes, such as fluidization. However, by the astounding growth in capability of computer modeling, it is merely possible to incorporate all terms of the governing equations and occurring scales in the simulations of complex processes and flows.

Speaking of accuracy in CFD applications reminds one of the direct numerical simulation (DNS) approach. DNS aims to resolve all the occurring temporal and spatial scales of turbulence in the flow and is considered as an advanced and accurate simulation tool which can be used to validate other numerical models (Xu and Subramaniam, 2006). In DNS, the hydrodynamic forces acting on the solid particles are directly computed from the fluid flow, and the motion of the fluid flow and solid particles are fully coupled, 
according to Wang et al., (2009) and Hu et al., (2001). The limitation of this approach is mainly the available computer power, which limits its applications to simulation of flows in small domains. With the increase of the parallel computing capabilities, it has become a possibility to perform the DNS modeling of processes happening in device scale domains. Yet, the time and storage requirements are still incomparable to other wellknown available techniques in the CFD of multiphase flows, such as two fluid modeling (TFM, van Deemter and van der Laan, 1961), discrete particle modeling (DPM, Tsuji et al. 1993), and Energy Minimization Multi-scale method (EMMS, Li and Kwauk, 1994, 2003). In an effective approach, one can decide to perform the device scale simulations using the TFM approach by implementing and employing precise sub-models which are obtained from direct numerical simulation or experimentation of the same flow configuration. Because of this, appreciable advantages are obtained in regards to the computational time and memory.

According to Xu and Subramaniam, (2006), utilization of the two-fluid approach relies on unclosing terms representing the interaction between the phases. Interaction between the phases is a crucial fact in simulation of the gas-solid fluidization flows involving cohesive particles. Empirical correlations based on experiments by Ergun (1952) and Wen-Yu (1966) have been used frequently to calculate the drag force in dilute and dense flow regimes. These drag laws are applicable to suspensions where particles do not form clusters, and they have been useful in modeling the hydrodynamics of fluidized beds for Geldart B and D particles (Wang, 2009). However, several studies have shown that CFD simulation of fluidized beds with Geldart A particles remains a challenge because they 
fail to reproduce the pressure drop and bed expansion in bubbling regime (Mckeen and Pugsley, 2003 and Zimmermann and Taghipour, 2005) and solid distribution along the fluidization column in fast fluidized regime (Hong et al., 2012).

The primary reason for the above mentioned failures is that the drag force is significantly overestimated by standard drag laws (Mckeen and Pugsley, 2003, Syamlal and T.J. O'Brien, 2003, and Zimmermann and Taghipour, 2005). Formation of particle clusters significantly reduces the drag force, which is confirmed by recent studies using particleresolved direct numerical simulation (DNS) of flow past fixed particle assemblies. Example of this practice can be found in the work of Koch and Sangani (1999 and 2001), Hill and Koch (2001a\&b), Garg et al. (2011), Yin and Sundaresan (2009-a\&b). Such precise studies have yielded drag relations that are more accurate than the Ergun and Wen-Yu correlations according to Bokkers and van Sint Annaland (2004), because they account for particle clustering phenomenon. However, as mentioned earlier, there are cost and time inhibitive reasons for using DNS-based modeling for the device-scale simulations. Ad hoc approaches such as EMMS model of Li and Kwauk (2003) were developed to account for the presence of particle clusters. However, these models were successful in improving the simulation results in a limited fluidization regime. Moreover, these ad hoc modifications provide no insight into the fundamental multiphase flow physics and may not have predictive capabilities over the parameter range that is necessary for design optimization. Therefore, a first-principle based approach that can quantify the mechanisms underlying particle clustering and their effect on the drag force must be pursued and evaluated. 
In this dissertation, it is hypothesized that:

1. Momentum exchange between the gas and solid significantly changes the distribution of solid particles along the fluidization column and consequently on the mass of solid exiting the column.

2. Formation of clusters significantly and directly influence the Momentum exchange coefficients between the solid and gas.

3. Implementing a cluster prediction method and correction of the drag force based on this prediction could improve solid concentration along the riser.

Therefore, the present dissertation focuses on the development and application of a numerical model that can 1) predict cluster formation for particles in Geldart A regime, 2) implement the effect of cluster formation on the momentum exchange coefficient for computational cells that are marked for clustering modification. Prediction of particle clustering relies on the mechanism of particle clustering. The effect of the clustering phenomenon on the flow simulation is imposed by coupling a standard drag model to a DNS-generated drag correlation. Development and application of this numerical model is performed in the Multiphase Flow with Interphase Exchanges (MFIX) CFD package aiming to simulate multiphase flows effected by clustered particles.

\subsection{Objectives}

The present $\mathrm{PhD}$ dissertation focuses on the following objectives:

- Developing a cluster prediction mechanism applicable to Two-Fluid Modeling of gas-solid systems. 
- Developing more accurate drag models for TFM simulation of gas-solid fluidization flow with presence of cohesive interparticle forces.

- Using the developed models to explore the improvement of accuracy of TFM in simulation of fluidization flow of cohesive powders.

- Understanding the causes and influential factors which led to improvements and quantification of improvements.

- Gathering data from a fast fluidization flow and use these data to compare with simulated results.

\subsection{Dissertation Organization}

The rest of this dissertation is organized as follows: Chapter 2, (published by the journal of Powder Technology), presents development of a hybrid model based on a particle resolved DNS model for Two-Fluid Modeling simulation of the fluidization of gas and particles in Geldart A regime. The concept of cohesive index and implementation of this concept in the drag modifications is introduced. This implementation targets a switching mechanism between the particle resolved DNS-based hybrid model and three standard drag models suitable for non-clustering particles. Benchmark validation of the model using available experimental data in the literature will be provided and contribution of each hybrid model in improvement of numerical accuracy will be quantified.

Chapter3, (to be submitted to the journal of Powder Technology), describes the effects of switching mechanism between the standard models and the particle resolved DNS based 
model. A thorough analysis is performed to determine the governing factors which contribute toward improvements of numerical results.

Chapter 4, (to be submitted to the journal of Powder Technology) describes the development of a cluster-aware hybrid drag model for Two-Fluid Modeling simulation of the fluidization of gas and particles in Geldart A regime. Benchmark validation of the model using available experimental data in the literature will be provided and contribution of the hybrid model toward improvement of numerical accuracy will be quantified.

Chapter 5, (to be submitted to the journal of Powder Technology) describes an experimental approach towards developing data for benchmark validation. This procedure involves obtaining the profile of pressure along the riser of a circulation fluidized bed developed at the Laboratory of Applied Research Center at FIU. This data can serve other researchers in the field of fluidization flow for their benchmark validation attempts. This chapter also investigates the performance of the cluster-aware drag model introduced in chapters 2 and 4 of this dissertation in simulation of the flow in this facility using the data from measurements. Finally, the dissertation concludes in Chapter 5 with a summary of the four main chapters and will suggest future research paths in this area.

\subsection{References}

Berutti $\mathrm{F}$ and Kalogerakis N. Modelling the internal flow structure of circulating fluidized beds. Can. J. Chem. Eng., Vol. 67, pp 1010-1014, 1989. 
Bokkers G. A., van Sint Annaland M. K. J., 2004, Mixing and segregation in a bidisperse gas-solid fluidised bed: a numerical and experimental study, Powder Technology Journal, Volume 140, pp. 176-186.

Geldart D., "Types of Gas Fluidization," 1972, Powder Technology 7, pp 285-292 (1972)

Cocco R. Shaffer F., Hays R.S.B., Karri R., Knowlton T., 2010, Particle clusters in and above fluidized beds, Journal of Powder Technology, Volume 203, pp 3-11

Cocco R., Shaffer F., Karri S.B.R., Hays R., Knowlton T., 2011, Particle Clusters In luidized Beds, ECI Conference on The 13th International Conference on Fluidization New Paradigm in Fluidization Engineering

Cocco R., Reddy Karri S.B., Knowlton T., 2014, Introduction to Fluidization, American Institute of Chemical Engineering,(AIChE), Issue CEP 2014

Fluidization Engineering, 2nd Edition from D. Kunii, Octave Levenspiel. ISBN9780409902334, Printbook, Release Date: 1991

Ergun S., 1952, Fluid flow through packed columns, Chemical Engineering Progress Symposium Series, Volume 48, Issue 2, pp 89-94.

Garg R., Tenneti S., Yusof J.M., 2011, and Subramaniam S., Direct Numerical Simulation of Gas-Solids Flow Based on the Immersed Boundary Method, Computational Gas-Solids Flows and Reacting Systems: Theory, Methods and Practice, IGI global disseminator of knowledge

Geldart D., 1972, Types of Gas Fluidization, Powder Technology Journal, Volume 7, pp 285-292

Hong K., Wang W., Zhou Q., Wang J., Li J., 2012, An EMMS-Based Multi-Fluid Model (EFM) for Heterogeneous Gas-Solid Riser Flows: Part I. Formulation of StructureDependent Conservation Equations, Journal of Chemical Engineering Science, Volume 75, pp 376-389

Hu H.H., Patankar N.A., Zhu, M.Y., 2001, Direct numerical simulations of fluid-solid systems using the arbitrary Lagrangian-Eulerian technique. Journal of Computational Physics, Volume 169, Issue 2, pp 427-462

Koch D.L., Sangani A.S., 1999, Particle pressure and marginal stability limits for a homogeneous monodisperse gas-fluidized bed: kinetic theory and numerical simulations, Journal of Fluid Mechanics, vol. 400, pp. 229-263 
D. L. Koch and R. J. Hill, 2001, Inertial Effects in Suspension and Porous-Media Flows, Annual Review of Fluid Mechanics, Volume 33, pp. 619-647.

Li H., Xia Y., Tung Y., Kwauk M., 1991, Micro-visualization of clusters in a fast fluidized bed, Powder Technology, Volume 66, pp 231-235

Li J., Kwauk M., 1994, Particle-Fluid Two Phase Flow, The Energy Minimization Multiscale Method, Metallurgy Industry Press, Beijing

Li J. and Kwauk M., 2003, Exploring complex systems in chemical engineering - the multi-scale methodology.,Chemical Engineering Science, Volume 58, Issue 3-6, pp. 521535

McKeen T., Pugsley T., 2003, Simulation and Experimental Validation of a Freely Bubbling Bed of FCC Catalyst, Journal of Powder Technology, Volume 129, Issues 1-3, pp 139-152

Syamlal M., O’Brien T. J., 2003, Fluid Dynamic Simulation of O3 Decomposition in 3 a Bubbling Fluidized Bed, AIChE Journal, Volume 49, Issue 11, pp 2793-2801

Tsuji Y., Kawaguchi T., Tanaka T., 1993, Discrete particle simulation of a fluidized bed. Powder Technology, Volume 77, Issue 1, pp 79-87

van Deemter, J. J., van der Laan, E. T., ,1961, Momentum and Energy Balances for Dispersed Two-Phase Flow, Journal of Applied Sciences Research, Section A, Volume 10, pp 102-108

Wen C. Y., Yu Y. H., 1966, Mechanics of Fluidization, Chemical Engineering Progress Symposium Series, Volume 62, Issue 1, pp 100-111

Wang J., 2009, A Review of Eulerian Simulation of Geldart A Particles in Gas-Fluidized Beds, Journal of Industrial and Engineering Chemistry Research, volume 48, Issue 12, pp 5567-5577

Xu Y. and Subramaniam S., A multiscale model for dilute turbulent gas-particle flows based on the equilibration of energy concept, Physics of Fluids 18, 033301 (2006); doi: $10.1063 / 1.2180289$, Ye et al

Xu J., Zhu J.X., 2011, Visualization of particle aggregation and effects of particle properties on cluster characteristics in a CFB riser, Chemical Engineering Journal, Volume 168, Issue 1, pp 376-389 
Ye M., van der Hoef M.A., Kuipers J.A.M., 2005a, From Discrete Particle Model To A Continuous Model of Geldart A Particles, Journal of Chemical Engineering Research and Design, Volume 83, Issue 7, pp 833-843

Ye M.A, van der Hoef M.A., Kuipers J.A.M., 2005b, The Effects of Particle and Gas Properties on the fluidization, Journal of Chemical Engineering Science, Volume 60, Issue 16, pp 4567- 4580

Ye M. A., van der Hoef M.A., Kuipers J.A.M, 2008, Two-fluid modeling of Geldart A particles in gas-fluidized beds, Journal of Particuology, Volume 6, Issue 6, pp 540-548

Yin X., Sundaresan S., 2009, Drag Law for Bidisperse Gas-Solid Suspensions Containing equally sized spheres, Journal of Industrial and Engineering Chemistry Research, Volume 48, Issue 1, pp 227-241

Yang T-Y, Lue L-P, Multiresolution Analysis on Identification and Dynamics of Clusters in a Circulating Fluidized Bed, American Institute of Chemical Engineers AIChE J, Volume 55: 612-629, 2009

Yerushalmi J., Gluckman M.J., Graff R.A., Dobner S., Squires A.M,, 1975, Production Of Gaseous Fuels From Coal In The Fast Fluidized Bed, Fluidization Technology, Volume 11

Yerushalmi J., Turner D.H, 1976, Squires A.M, The Fast Fluidized Bed, Journal of Industrial Engineering Chemistry, Volume 15, issue 47, No. 1

Zimmermann s. and Taghipour F., 2005, CFD Modeling Of The Hydrodynamics And Reaction Kinetics Of FCC Fluidized-Bed Reactors., Industrial \& Engineering Chemistry Research, vol. 44, no. 26, pp. 9818- 9827, 2005. 
CHAPTER 2: IMPROVEMENT OF EXISTING STANDARD TWO-FLUID DRAG MODELS IN SIMULATION OF GAS-SOLID FLOWS USING A PR-DNS DRAG MODEL

\subsection{Abstract}

This study investigates a new drag model for the simulation of the fluidization of fluid catalytic cracking (FCC) particles with air in a fluidized bed using the two-fluid model (TFM) within the Multiphase Flow with Interphase Exchanges (MFIX) code. A cohesion index parameter based on the interparticle cohesive forces has been implemented in the MFIX-TFM code. This index is used as a switching criterion between a particle resolved drag model developed by Tenneti et al. (2011), and some of the drag models available in the MFIX for homogeneous particles, namely the Gidaspow, Syam-O'brien, and WenYu models. The proposed drag correlation in this paper implements an indirect method of introducing interparticle cohesive forces to our TFM simulations. Significant improvement in the solid volume fraction profile along the riser was obtained for all of the drag law combinations, depending on the conditions set in the switching procedures. In the best case, the utilization of the Gidaspow and TGS models resulted in a $60 \%$ improvement in maximum deviation of numerical results from the available experimental data. The proposed model can be used in simulations of fluidized beds, where standard models fail to produce accurate results even on extremely refined computational grid, especially for Geldart A type particles that may exhibit strong clustering behavior. 


\subsection{Introduction}

The TFM approach, developed by van Deemter and van der Laan (1961), is known as an economic way of simulating multiphase flows in large-scale fluidized bed risers. Formulating the solid and gas as continuous phases is the principle of the TFM method. This leads to significant reduction of memory and computational costs as compared to other widely exploited methods, such as the Particle-Resolved Direct Numerical Simulation (Hu et al., 2001 and Nomura and Hughes, 1992), Discrete Element Method (Tsuji et al. 1993), and structure-based methods, such as the Discrete Bubble Model (Bokkers et al. 2006), and the Discrete Cluster Model (Liu et al., 2006 and Zou et al. 2008).

One notable drawback of the TFM in MFIX is the absence of cohesive inter-particular forces, such as electrostatic and van der Waals forces between particles. These forces play a major role in fluidization of strongly cohesive particles in Geldart A and C groups by creating heterogeneous structures, called clusters. According to Li et al. (2013), clusters affect the flow significantly by changing the mass and momentum transfers between the gas and solid phases. Many researchers, such as Andrews et al. (2005), Agrawal et al. (2001), Zhang and Vanderheyden (2002), McKeen and Pugsley (2003), Yang et al. (2003), Ye et al. (2005 a-b and 2008), Qi et al. (2007), Wang et al.(2008), Wang and Li (2007),Wang (2009), Lu et al.(2009), Igci et al. (2012), and Li et al. (2013), believe that clusters are responsible for significant reduction of the interfacial drag forces between the gas and solid phases. Therefore, dependency of the drag forces on the nature of the attractive interparticle forces plays as important role as the 
dependency on two other parameters, i.e., the Reynolds number of the flow around particles and the volume fraction of the solid phase in each computational cell.

There have been several attempts to improve the performance of the MFIX-TFM code by introducing more complex drag laws, which can consider the effect of subgrid-scale heterogeneous structures in TFM simulations, such as the filtered models of Igci et al. (2012) and Milioli et al. (2013) and Andrews et al. (2005), according to van der Hoef et al. (2008) and Wang et al. (2009). However, the constitutive models used in these filtered models were obtained from highly resolved simulations of kinetic theory-based TFM simulations in the absence of the cohesive interparticle forces. This gap can be filled by inclusion of cohesive interparticle forces in the MFIX-TFM code, similar to the inclusion of van der Waals in the MFIXDEM code (MFIX-2013 Release Notes). In addition, no study has been found in the literature that has implemented the inclusion of the van der Waals forces in the drag laws within the MFIX-TFM code.

DNS has been widely used in high resolution simulation of gas- particle flows in suspension and fluidized beds by researchers such as Ma et al. (2006), Cho et al.(2005), Xiong et al. (2012), and Yin and Sundaresan (2009), Garget et al. (2011) and Sharma and Patankar(2005). Ma et al. (2006) acknowledged the diversity and structural dependence of the drag force on each particle, rather than relying on the entire control volume performed in methods such as TFM. Their analysis, akin to DNS analysis of Xiong et al. (2012), proved that the drag force is significantly different on particles in dilute regions compared to grouped particles. One useful approach in DNS modeling is 
the analysis of the flow over fixed assemblies of particles, as practiced by Hill et al. (2001, a-b), van der Hoef et al. (2005), Beetstra et al. (2007), Yin and Sundaresan (2009), and Tenneti and Subramaniam (2011). This approach increases the accuracy and relevance of the information collected. For example, information about field variables, such as the coefficient of drag, and gas and particle velocities can be obtained. Additionally, various different cluster configurations could be analyzed. Cluster differences include: shape, compactness, orientation of the cluster relative to the fluid, spinning speed of the cluster, and various flow-solid relative velocities. A combined particle or cluster resolved DNS analysis coupled with the TFM analysis of the flow could contribute to the improvement of the TFM modeling of the clustering multiphase flow systems. There is also an opportunity for a simulation of the flow on the industrialscale, using the information obtained in particle or cluster-scale.

Presently, MFIX is a widely known, reliable, and professionally established package for simulation of heat and mass transfer. MFIX accommodates a variety of drag models that can be used in TFM simulation of gas-solid particulate flows. Yet, the direct or indirect addition of models for particle-to-particle, attractive and repulsive forces to the transport equations solved in TFM, or to the available drag laws, is missing. According to Ye et al. (2005, a-b) and Seville et al. (2000), these forces could be formulated as $\overrightarrow{\mathrm{F}}_{\mathrm{ij}}(\mathrm{c})=$ $\left(A R / 6 d_{i j}{ }^{2}\right) \vec{n}_{i j}$ where $F_{i j}{ }^{(c)}$ is the cohesive inter-particular force and A is the Hamaker constant ( $\left.\approx 10^{-19} \mathrm{~J}\right)$ (1991), $\mathrm{R}$ is the radius of the mono-dispersed particles, $\mathrm{d}$ is the surface to surface distance between particles and $\vec{n}_{i j}$ is the normal vector pointing from the center of particles $\mathrm{i}$ to the center of the particle $\mathrm{j}$. Further, they defined a scaling factor, 
$\varphi=\frac{\left|U_{\min }\right|}{K_{B} T}=\frac{A R}{6 z_{0}} \cdot \frac{1}{K_{B} \Theta_{S}}$, which is the ratio between the interparticle cohesive and destabilizing forces for $\mathrm{d} \leq 100 \mu \mathrm{m}$. In this definition, $\mathrm{K}_{\mathrm{B}}$ is the Boltzmann constant ( $\mathrm{K}_{\mathrm{B}} \approx 1$, Ye et al, 2005), $\mathrm{Z}_{0}$ is the threshold for particles to be considered as clustered $\left(\mathrm{Z}_{0}\right.$ $\approx 4 \mathrm{~nm}$, Seville et al., 2005) and $\mathrm{d}$ and $\Theta$ s are the diameter and granular temperature of the solid particles. The derivation of equations governing the particle motion can produce a similar quantifying scaling factor, which can indicate the onset of cluster formation. In this analysis, as compared to the cohesion models available in the MFIX-DEM, the scaling factor is an additional factor to be considered for cluster formation, (in addition to the surface to surface particle distances).

Destabilizing forces in the particle-gas systems are mainly due to the particle-to-particle and particle-to-gas interactions. These interactions significantly influence the analysis of particle-gas flows, which has attracted the attention of many researchers, such as Dombrowski and Johns (1963), Gidaspow (1994), Ding and Gidaspow (1990), Cho et al. (2005), Benyahiah (2012), Karimipour and Pugsley (2012), and Syamlal et al. (2013). Special attention has been paid to this parameter in the work of Yet et al.(2005-a). The granular temperature is a measure of the particle fluctuating energy and could be used as a critical parameter to predict the coalescence of particles and break-up of clusters in numerical simulations. MFIX-TFM can solve the transport equation or the algebraic equation, in order to obtain the granular temperature. 
In this study, we introduce a cohesive index into the MFIX-TFM code and implement it as a criterion for switching between a Particle- Resolved Direct Numerical-Simulation model, the TGS model, and three existing drag models available in the MFIX code. The rest of the paper is organized as follows. First, the model formulation is presented where the governing equations for the TFM model, the governing equations related to the model of motion of particles leading to our cohesive index and the governing equations of the Gidaspow, Syam- O'Brien, Wen-Yu and TGS drag models are presented. Later, the methodologies for implementing the cohesive index, error calculations and switching between the TGS and other models is presented and followed by the examination of the proposed models in numerical simulations for flow in a fluidized bed. Finally, a conclusion is drawn on the effectiveness of the proposed model and the authors' perspective of the future work.

\subsection{Governing Equations}

In the TFM, both the gas and the particulate phases are considered as interpenetrating continuous mediums. Complete derivations of the equations governing the two-fluid model can be found in the work of Gidaspow (1994). Here, the equations of the TFM model for flow without phase change and chemical reactions are given by Samuelsberg and Hjertager (1996) as

$$
\begin{gathered}
\frac{\partial\left(\rho_{\mathrm{k}} \varepsilon_{\mathrm{k}}\right)}{\partial \mathrm{t}}+\nabla \cdot\left(\rho_{\mathrm{k}} \varepsilon_{\mathrm{k}} \overrightarrow{\mathrm{u}}_{\mathrm{k}}\right)=0, \\
\frac{\partial\left(\rho_{\mathrm{k}} \varepsilon_{\mathrm{k}} \overrightarrow{\mathrm{u}}_{\mathrm{k}}\right)}{\partial \mathrm{t}}+\nabla \cdot\left(\rho_{\mathrm{k}} \varepsilon_{\mathrm{k}} \overrightarrow{\mathrm{u}}_{\mathrm{k}} \overrightarrow{\mathrm{u}}_{\mathrm{k}}\right)=-\varepsilon_{\mathrm{k}} \nabla \mathrm{p}_{\mathrm{k}}+\varepsilon_{\mathrm{k}} \rho_{\mathrm{k}} \overrightarrow{\mathrm{g}}+\nabla \cdot\left(\varepsilon_{\mathrm{k}} \overline{\overline{\mathrm{t}}}_{\mathrm{k}}\right)+\beta\left(\overrightarrow{\mathrm{u}}_{\mathrm{l}}-\overrightarrow{\mathrm{u}}_{\mathrm{k}}\right),
\end{gathered}
$$




$$
\overline{\bar{\tau}}_{\mathrm{k}}=\left[\lambda_{\mathrm{k}} \nabla \cdot \overrightarrow{\mathrm{u}}_{\mathrm{k}}\right] \overline{\overline{\mathrm{I}}}+\mu_{\mathrm{k}}\left[\nabla \overrightarrow{\mathrm{u}}_{\mathrm{k}}+\left(\nabla \overrightarrow{\mathrm{u}}_{\mathrm{k}}\right)^{\mathrm{T}}\right] .
$$

Eqs. (2.1) to (2.3) show the equations for continuity, momentum balance, and the stress tensor for the phases in TFM, respectively. In these equations, $\rho, \overrightarrow{\mathrm{u}}, \varepsilon, \mathrm{g}, \overline{\bar{\tau}}_{\mathrm{k}}, \beta, \mathrm{p}, \lambda$ and $\mu$ represent the density, velocity vector, volume fraction, acceleration of gravity, shear stress tensor, momentum exchange coefficient, thermodynamic pressure, second coefficient of viscosity (or bulk viscosity), and the dynamic viscosity of the phases. In addition, $\mathrm{k}$ and $\mathrm{l}$ serve as identifiers for gas and solid phases. However, in Eq. (2.2), identifiers are phase specific, where if $\mathrm{k}$ refers to one of the phases (e.g., fluid), then $\mathrm{l}$ can only refer to the solid, and vice versa. In this work, the second coefficient of viscosity for the gas phase is set to zero, as suggested by Lu et al. (2009). The pressure term for the solid phase $\left(\mathrm{p}_{\mathrm{S}}\right)$ is obtained by grouping the gas pressure and the solid phase pressure together, as displayed by Eq. (2.4).

$$
\mathrm{p}_{\mathrm{s}}=\mathrm{pg}_{\mathrm{g}}+\mathrm{P}_{\mathrm{s}}
$$

The solid phase pressure is obtained from the granular kinetic model of Ding and Gidaspow (1990), as $P_{S}=\Theta_{s}\left[1+2\left(1+e_{s s}\right) \varepsilon_{s} g_{0 s s}\right]$. Where, $\Theta_{s}$ and $e_{s s}$ represent the granular temperature of the solid phase and particle-particle restitution coefficient, respectively. Here, the $\mathrm{e}_{\mathrm{ss}}$ is set to 0.9 according to Jenkins and Zhang (2002) and Benyahia (2012). In addition, the solid bulk viscosity, solid shear viscosity, and radial distribution function are given by Samuelsberg and Hjertager (1996) as 


$$
\begin{gathered}
\lambda_{\mathrm{s}}=\rho_{\mathrm{s}} \mathrm{d}_{\mathrm{p}}\left(\mathrm{e}_{\mathrm{sS}}+1\right) \frac{4 \varepsilon_{\mathrm{s}}^{2} \sqrt{\Theta_{\mathrm{s}}} \mathrm{g}_{0}}{3 \sqrt{\pi}}, \\
\mu_{\mathrm{s}}=\frac{5 \sqrt{\left(\pi \Theta_{\mathrm{s}}\right)} \rho_{\mathrm{s}} \mathrm{d}_{\mathrm{p}}}{48\left(\mathrm{e}_{\mathrm{ss}}+1\right) \mathrm{g}_{0}}\left[\left(1+\frac{4}{5}\left(\mathrm{e}_{\mathrm{ss}}+1\right) \varepsilon_{\mathrm{s}} \mathrm{g}_{0}\right]+\left(\frac{2 \varepsilon_{\mathrm{s}}^{2} \rho_{\mathrm{s}} \mathrm{d}_{\mathrm{p}} \mathrm{g}_{0}\left(1+\mathrm{e}_{\mathrm{ss}}\right) \sqrt{\Theta_{\mathrm{s}}}}{5 \sqrt{\pi}}\right),\right. \\
\mathrm{g}_{0}=\frac{3}{5}\left[1-\left[\frac{\varepsilon_{\mathrm{s}}}{\varepsilon_{\mathrm{S}_{\max }}}\right]^{1 / 3}\right]^{-1},
\end{gathered}
$$

respectively. The transport equation for the granular energy is originally derived by Ding and Gidaspow (1990). However, a more complete version is given by Lu et al. (2009) as

$$
\begin{aligned}
& \frac{3}{2}\left[\frac{\partial}{\partial \mathrm{t}}\left(\rho_{\mathrm{s}} \varepsilon_{\mathrm{S}} \Theta_{\mathrm{S}}\right)+\nabla \cdot\left(\rho_{\mathrm{s}} \varepsilon_{\mathrm{S}} \overrightarrow{\mathrm{v}}_{\mathrm{s}} \Theta_{\mathrm{S}}\right)\right]=\left(-\varepsilon_{\mathrm{S}} P_{\mathrm{S}} \overline{\overline{\mathrm{I}}}+\varepsilon_{\mathrm{S}} \overline{\overline{\mathrm{s}}}_{\mathrm{S}}\right): \nabla \overrightarrow{\mathrm{u}}_{\mathrm{s}}-\nabla \cdot\left(\mathrm{k}_{\theta_{\mathrm{S}}} \nabla \theta_{\mathrm{S}}\right)- \\
& \gamma_{\theta_{\mathrm{s}}}-3 \beta \Theta_{\mathrm{S}} .
\end{aligned}
$$

In this equation $\Theta_{\mathrm{s}}$ is the granular temperature. The diffusion coefficient of the granular energy and the collisional energy dissipation are defined by Eq. (2.9) and Eq. (2.10), respectively (Samuelsberg and Hjertager, 1996).

$$
\begin{gathered}
\mathrm{k}_{\boldsymbol{\theta}_{\mathrm{S}}}=\frac{150 \rho_{\mathrm{S}} \mathrm{d}_{\mathrm{s}} \sqrt{\left(\Theta_{\mathrm{s}} \pi\right)}}{384\left(1+\mathrm{e}_{\mathrm{SS}}\right) \mathrm{g}_{0}}\left[1+\frac{6}{5} \varepsilon_{\mathrm{s}} \mathrm{g}_{0}\left(1+\mathrm{e}_{\mathrm{ss}}\right)\right]^{2}+2 \rho_{\mathrm{s}} \varepsilon_{\mathrm{s}}^{2} \mathrm{~d}_{\mathrm{s}}\left(1+\mathrm{e}_{\mathrm{sS}}\right) \mathrm{g}_{0} \sqrt{\frac{\Theta_{\mathrm{s}}}{\pi}} \\
\gamma_{\theta_{\mathrm{s}}}=3\left(1-\mathrm{e}_{\mathrm{ss}}{ }^{2}\right) \rho_{\mathrm{s}} \varepsilon_{\mathrm{s}}{ }^{2} \Theta_{\mathrm{s}} \mathrm{g}_{0}\left(\frac{4 \sqrt{\Theta_{\mathrm{S}}}}{\mathrm{d}_{\mathrm{s}} \sqrt{\pi}}-\nabla \cdot \overrightarrow{\mathrm{u}}_{\mathrm{s}}\right) .
\end{gathered}
$$


According to Ye et al. (2005), the translational state of the particulate phase is described by the Newtonian equations of motion, i.e.. Eq. (2.11), for each individual particle in the system.

$$
m_{i} \frac{d^{2} \vec{x}_{i}}{d t^{2}}=\vec{F}_{c, i}+\vec{F}_{v d w, i}+\vec{F}_{d r a g, i}-V_{i} \nabla p+m_{i} \vec{g}
$$

The terms on the RHS of this equation are the contact force, the van der Waals force, the gas-particle drag force, the force due to pressure gradient in the fluid, and the gravitational force, respectively. In these terms, $\mathrm{m}$ is the mass of the particle, $\mathrm{V}$ is the particle volume, $\vec{x}$ is the position vector pointing from the center of the particle $\mathrm{j}$ to the center of the particle $i$, and $\vec{g}$ is the vector of acceleration of gravity. Eq. (2.11) can be rewritten for particle $\mathrm{j}$ with exactly the same properties as the particle $\mathrm{i}$ where, the surfaces of the two particles, $i$ and $j$, are assumed to be in small separation distance from each other. In our approach, since particles are not in a contact, the collisional force term, $\overrightarrow{\mathrm{F}}_{\mathrm{c}, \mathrm{i}}$, vanishes from our equation. Later, by considering the small size and separation distance of the particles, with a good estimate, we assume that particles are affected equally by the gas flow and the gravity field variables. Hence, the third, fourth, and the fifth terms on the RHS are assumed equal in magnitude and direction. However, particles $\mathrm{i}$ and $\mathrm{j}$ exchange equal van der Waals forces in opposite directions. Here, we use the expression of Ye et al. (2005) for the van der Waals force between two identical spherical particles and obtain a simplified form for the relative equation of translational motion for particles, as follows

$$
m_{\text {eff }} \frac{\mathrm{d}^{2} \overrightarrow{\mathrm{x}}_{\mathrm{ij}}}{\mathrm{dt}^{2}}+\frac{\mathrm{AR}_{\mathrm{s}}}{6 \mathrm{~d}^{2}}=0
$$


Where, $\overrightarrow{\mathrm{x}}_{\mathrm{ij}}$ is the vector of instantaneous relative position of the particle $\mathrm{i}$ with respect to the particle $\mathrm{j}$, meff is the effective mass defined as $\mathrm{m}_{\text {eff }}=\mathrm{m}_{\mathrm{i}} \mathrm{m}_{\mathrm{j}} /\left(\mathrm{m}_{\mathrm{i}}+\mathrm{m}_{\mathrm{j}}\right)$, and $\mathrm{A}, \mathrm{R}_{\mathrm{s}}$ and, d are the Hamaker constant, radius of the particle, and the separation distance between the two particles, respectively. Complete derivation of the governing equations is available in Appendix A. Later, definition of the granular temperature, $\Theta=1 / 3$ 〈particle velocity fluctuation $\left.{ }^{2}\right\rangle$ (Lun et al.,1984and Gidaspow, 1994), and a short range separation distance within which attractive forces are dominant (Ye et al. , 2005-a), do, are used to create dimensionless parameters, such as $\tilde{t}=t \sqrt{\Theta_{S}} / d_{0}$ and $\overrightarrow{\widetilde{X}}_{i j}=\vec{X}_{i j} / d_{0}$, respectively. By defining the vector of relative velocity as $\vec{V}^{(i j)}=d \vec{x}^{(i j)} / d t$, we obtain

$$
\mathrm{Ha}^{-1}\left[\frac{\mathrm{d}_{0}}{\mathrm{~d}_{\mathrm{p}}}\right] \frac{\mathrm{d} \overrightarrow{\widetilde{\mathrm{V}}}_{\mathrm{ij}}}{\mathrm{d} \tilde{\mathrm{t}}}+1=0,
$$

where, $\widetilde{\vec{V}}^{(i)}=\overrightarrow{\mathrm{V}}^{(\mathrm{i})} / \sqrt{\Theta_{\mathrm{S}}}$ and the Ha parameter is defined as

$$
\mathrm{Ha}=\frac{\mathrm{A}}{\pi \rho \mathrm{d}_{\mathrm{p}}^{2} \mathrm{~d}_{0} \Theta_{\mathrm{S}}} .
$$

In this expression, $\rho$ is the density of the solid particle, $d_{p}$ is the particle diameter, $d_{0}$ is the surface to surface cut-off distance, and $\Theta$ is the granular temperature. The Ha parameter is the ratio of interparticle cohesive force to the destabilizing force, kinetic energy, acting on each particle in the computational domain. In our TFM simulations, the Ha parameter can be obtained for each computational node for the continuous representation of the solid phase. In fact, an increase of this parameter increases the chance of clustering in the domain. This heterogeneity discourages the use of the standard 
models which are appropriate for non-clustered particles. Therefore, it is appropriate to switch to a DNS-based drag model that can resolve the flow around small-scale structures, such as clusters of particles, more robustly for large values of Ha. Thus, we use this cohesive index as a criterion for switching between the TGS and other standard drag models. This idea is conceptualized in a new drag model, as illustrated in Table 2-1.

Table 2-1 Proposed drag model scheme using the cohesive index for MFIX-TFM simulations.

\begin{tabular}{lc}
\hline New drag model & \multicolumn{1}{c}{ Criteria } \\
\hline Use TGS & Ha $>$ Ha_threshold \\
Use an existing model & Ha $\leq$ Ha_threshold \\
\hline
\end{tabular}

The TGS drag model was developed by Tenneti et al. (2011) based on their immersed boundary method of the flow around fixed assemblies of particles (Tenneti and Subramaniam, 2013). TGS model with its improved correlation for the gas-solid drag force generates more accurate results for the same ranges of the flow Reynolds number and solid volume fraction compared to its succeeding particle resolved-DNS models. Moreover, TGS model extends the accuracy in DNS modeling of the gas- solid flows to include wider ranges of $\varepsilon s$ and Rem. Theoretically, The TGS model, displayed by Eq. (2.15), adds two modifications to the single particle-based drag law of Schiller and Naumann (1935), which is displayed by Eq. (2.16). These terms, defined as $F \varepsilon_{s}$ and $F \varepsilon_{s}, R_{p}$, include the pure effect of the solid volume fraction (Eq. 2.17), and, the combined effect of the Reynolds number and solid volume fraction (Eq. 2.18), respectively. The outcome from this model is the exchange coefficient defined by Eq. (2.19). 


$$
\begin{aligned}
& \mathrm{F}\left(\varepsilon_{\mathrm{S}}, \operatorname{Re}_{\mathrm{p}}\right)=\frac{\mathrm{F}_{\text {isol }}\left(\mathrm{Re}_{\mathrm{p}}\right)}{\left(1-\varepsilon_{\mathrm{S}}\right)^{3}}+\mathrm{F}_{\varepsilon_{\mathrm{S}}}\left(\varepsilon_{\mathrm{S}}\right)+\mathrm{F}_{\varepsilon_{\mathrm{S}}, \operatorname{Re}_{\mathrm{p}}}\left(\varepsilon_{\mathrm{S}}, \operatorname{Re}_{\mathrm{p}}\right) \\
& F_{\text {isol }}\left(\operatorname{Re}_{\mathrm{p}}\right)=1+0.15 \operatorname{Re}_{\mathrm{p}}{ }^{0.687} \text {, } \\
& \mathrm{F}_{\varepsilon_{\mathrm{S}}}\left(\varepsilon_{\mathrm{S}}\right)=\frac{5.81 \varepsilon_{\mathrm{S}}}{\left(1-\varepsilon_{\mathrm{S}}\right)^{3}}+0.48 \frac{\varepsilon_{\mathrm{S}}^{1 / 3}}{\left(1-\varepsilon_{\mathrm{S}}\right)^{4}}
\end{aligned}
$$

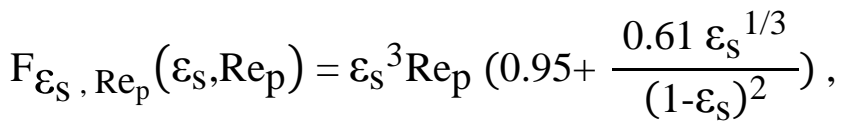

$$
\begin{aligned}
& \beta=18 \mu_{\mathrm{g}} \varepsilon_{\mathrm{g}} \varepsilon_{\mathrm{S}} \frac{\mathrm{F}\left(\varepsilon_{\mathrm{S}}, \mathrm{Re}_{\mathrm{p}}\right)}{\mathrm{d}_{\mathrm{p}}^{2}} .
\end{aligned}
$$

Table 2-2 shows different versions of the exchange coefficient $(\beta)$ in Equations (2.2) and (2.8), which are used in the existing drag models in MFIX. In addition, Table 2-3 shows the description of different versions of the drag model scheme labeled as AGDSM1, AGDSM2, and AGDSM3, as proposed in this work.

Table 2-2 Governing equations for the existing drag models used for switching procedure

O’Brien-Syamlal Drag Model (Syamlal et al., 1993, and Syamlal and O’Brien, 2003)

$$
\begin{aligned}
& \beta=\frac{3 \varepsilon_{\mathrm{g}} \varepsilon_{\mathrm{S}} \rho_{\mathrm{g}}}{4 \mathrm{~V}_{\mathrm{rs}}{ }^{2} \mathrm{~d}_{\mathrm{p}}} \mathrm{C}_{\mathrm{d} 0}\left(\frac{\mathrm{Re}_{\mathrm{p}}}{\mathrm{V}_{\mathrm{rs}}}\right)\left|\mathrm{V}_{\mathrm{g}}-\mathrm{V}_{\mathrm{s}}\right| \\
& \mathrm{V}_{\mathrm{rs}}=0.5\left(\mathrm{~A}-0.06 \mathrm{Re}_{\mathrm{p}}+\sqrt{\left(0.06 \mathrm{Re}_{\mathrm{p}}\right)^{2}+0.12 \mathrm{Re}_{\mathrm{p}}(2 \mathrm{~B}-\mathrm{A})+\mathrm{A}^{2}}\right. \\
& \mathrm{A}=\varepsilon_{\mathrm{g}}^{4.14} \\
& \mathrm{~B}=\left\{\begin{array}{lll}
0.8 \varepsilon_{\mathrm{g}}^{1.28} & \text { for } \quad & \varepsilon_{\mathrm{g}} \leq 0.85 \\
\varepsilon_{\mathrm{g}}^{2.65} & \text { for } & \varepsilon_{\mathrm{g}}>0.85
\end{array}\right.
\end{aligned}
$$




$$
\mathrm{C}_{\mathrm{d} 0}\left(\frac{\mathrm{Re}_{\mathrm{p}}}{\mathrm{V}_{\mathrm{rs}}}\right)=\left(0.63 \sqrt{\frac{\mathrm{Re}_{\mathrm{p}}}{\mathrm{V}_{\mathrm{rs}}}}+4.8\right)^{2} \times \frac{\mathrm{V}_{\mathrm{rs}}}{\mathrm{Re}_{\mathrm{p}}}
$$

Gidaspow Drag Model (Gidaspow (1994))

$$
\begin{aligned}
& \beta=\left\{\begin{array}{lll}
150 \frac{\varepsilon_{\mathrm{s}}^{2} \mu_{\mathrm{g}}}{\mathrm{d}_{\mathrm{p}}^{2} \varepsilon_{\mathrm{g}}}+1.75 \varepsilon_{\mathrm{s}} \frac{\rho_{\mathrm{g}}}{\mathrm{d}_{\mathrm{p}}}\left|\mathrm{V}_{\mathrm{g}}-\mathrm{V}_{\mathrm{s}}\right| & \text { for } & \varepsilon_{\mathrm{g}} \leq 0.8 \\
0.75 \mathrm{C}_{\mathrm{d} 0} \frac{\varepsilon_{\mathrm{s}}}{\varepsilon_{\mathrm{g}} \rho_{\mathrm{g}}} \mathrm{V}_{\mathrm{g}}-\mathrm{V}_{\mathrm{s}} \mid \varepsilon_{\mathrm{g}}{ }^{-2.65} & \text { for } & \varepsilon_{\mathrm{g}}>0.8
\end{array}\right. \\
& \mathrm{C}_{\mathrm{d} 0}=\left\{\begin{array}{lll}
0.44 & \text { for } & \mathrm{Re} \geq 1000 \\
\frac{24}{\operatorname{Re}_{\mathrm{p}}}\left(1+0.15 \mathrm{Re}_{\mathrm{p}}^{0.687}\right) & \text { for } & \mathrm{Re}<1000
\end{array}\right.
\end{aligned}
$$

Wen-Yu Drag Model (Wen and Yu,1966 and Xu et al., 2012)

$$
\begin{aligned}
& \beta=0.75 \mathrm{C}_{\mathrm{d} 0} \frac{\varepsilon_{\mathrm{S}} \varepsilon_{\mathrm{g}} \rho_{\mathrm{g}}}{\mathrm{d}_{\mathrm{p}}}\left|\mathrm{V}_{\mathrm{g}}-\mathrm{V}_{\mathrm{s}}\right| \varepsilon_{\mathrm{g}}-2.65 \\
& \mathrm{C}_{\mathrm{d} 0}=\left\{\begin{array}{lll}
0.44 & \text { for } & \mathrm{Re} \geq 1000 \\
\frac{24}{\mathrm{Re}_{\mathrm{p}}}\left(1+0.15 \mathrm{Re}_{\mathrm{p}}{ }^{0.687}\right) & \text { for } & \mathrm{Re}<1000
\end{array}\right.
\end{aligned}
$$

Table 2-3 Drag model combinations tested in this study.

\begin{tabular}{ccc}
\hline Version of the modelSwitching procedureCriteria for switching \\
\hline \multirow{2}{*}{ AGDSM 1} & Use TGS & $\mathrm{Ha}>\mathrm{Ha}$ _threshold \\
& Use Syam-O’Brien & $\mathrm{Ha} \leq$ Ha_threshold \\
\hline
\end{tabular}




\begin{tabular}{ccc}
\hline \multirow{2}{*}{ AGDSM 2} & Use TGS & Ha $>$ Ha_threshold \\
& Use Wen-Yu & Ha $\leq$ Ha_threshold \\
\hline \multirow{2}{*}{ AGDSM 3} & Use TGS & Ha $>$ Ha_threshold \\
& Use Gidaspow & Ha $\leq$ Ha_threshold \\
\hline
\end{tabular}

\subsection{Simulation Methodology}

The performance of each individual drag model was evaluated initially for a 20-second simulation of the flow in a circulating fluidized bed, as shown in Figure 2-1. We obtained permission from Li and Kwauk (1994) and Hong et al. (2012) to use their experimental and numerical data in our analysis, respectively. Hong et al. (2012) showed that utilization of the homogenous drag model, the correlation of Gidaspow (1994), fails to produce accurate grid-independent results. Similar failure was reported by $\mathrm{Lu}$ et al. (2009) and Benyahia (2008 and 2012) with the Ergun/Wen and Yu drag correlation (Ergun, 1952 and Wen and Yu, 1966). In the present study, similar to the work of Hong et al. (2012), we used the laminar flow assumption for the air and the transport equation of the granular temperature for fluctuations of the solid phase. However, in order to improve the accuracy in all simulation cases, we assigned the equation of the state for calculation of the air density. Settings are displayed in Table 2-4 


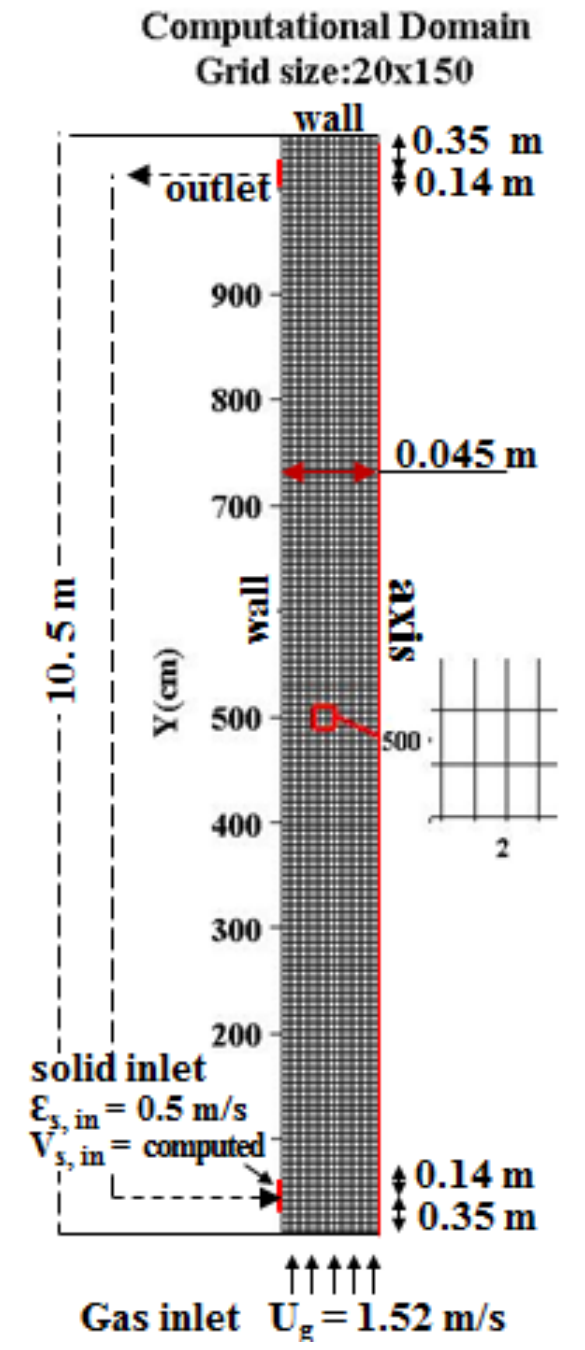

(a)

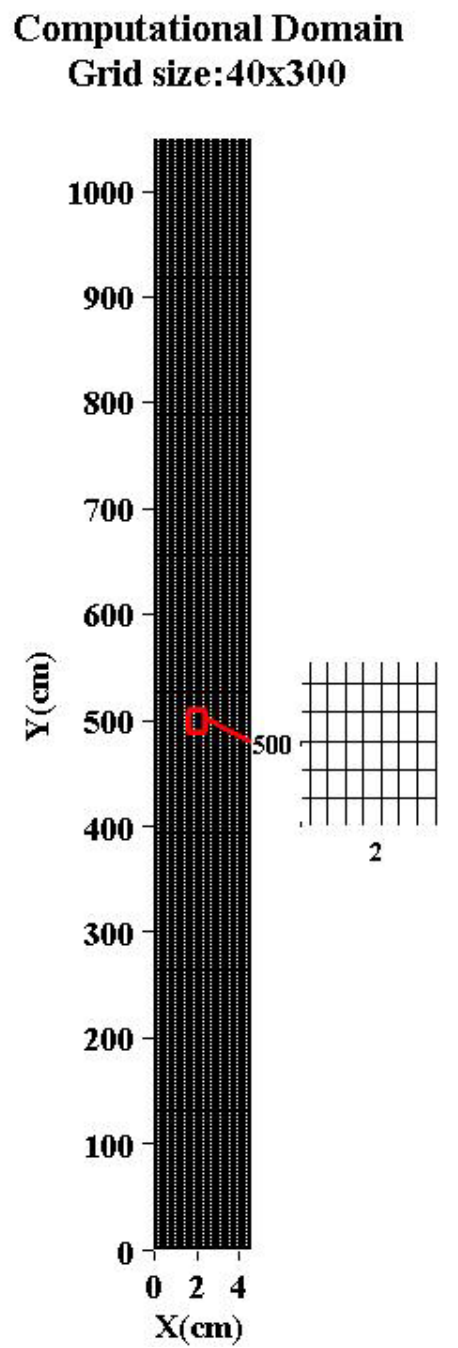

(b)

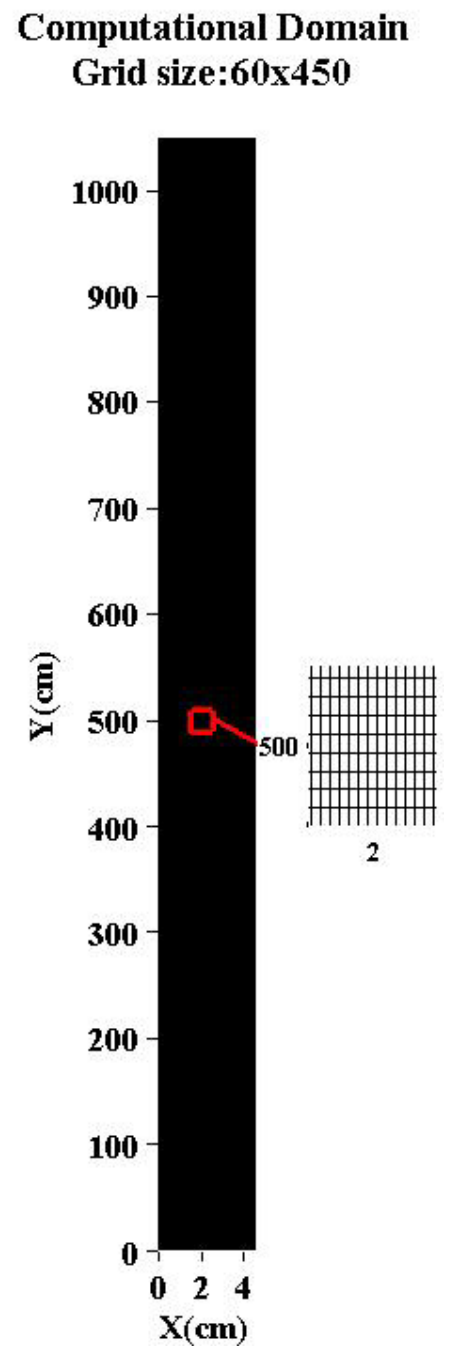

(c)

Figure 2-1 Computational domain and boundary conditions used for MFIX-TFM fluidized bed flow simulations, (a) $20 \times 150$, (b) $40 \times 300$, (c) $60 \times 450$ 
Table 2-4 Set up parameters for MFIX_TFM simulations.

\begin{tabular}{llll}
\hline $\begin{array}{l}\text { Property } \\
\text { Material }\end{array}$ & symbol & $\begin{array}{c}\text { value } \\
\text { air and FCC }\end{array}$ & unit \\
Particle diameter & $\mathrm{d}_{\mathrm{p}}$ & 54 & $\mu \mathrm{m}$ \\
Particle density & $\mathrm{Ro}_{\mathrm{s}}$ & 930 & $\mathrm{~kg} / \mathrm{m}^{3}$ \\
Air viscosity & $\mu_{\mathrm{g}}$ & $1.887 * 10^{-5}$ & $\mathrm{~Pa} . \mathrm{s}$ \\
Superficial gas velocity & $\mathrm{U}_{\mathrm{g}}$ & 1.52 & $\mathrm{~m} / \mathrm{s}$ \\
Solids mass flux & $\mathrm{G}_{\mathrm{s}}$ & 14.3 & $\mathrm{~kg} /\left(\mathrm{m}^{2} \mathrm{~s}\right)$ \\
Single particle terminal velocity & $\mathrm{u}_{\mathrm{t}}$ & 0.077 & $\mathrm{~m} / \mathrm{s}$ \\
Minimum fluidization voidage & $\varepsilon_{\mathrm{mf}}$ & 0.4 & - \\
Packing limit & $\varepsilon_{\mathrm{s}-\mathrm{max}}$ & 0.63 & - \\
Particle-particle coefficient restitution & $\mathrm{e}_{\mathrm{s}}$ & 0.9 & - \\
Particle-wall coefficient restitution & $\mathrm{e}_{\mathrm{w}}$ & 0.99 & - \\
Specularity coefficient & $\varphi$ & 0.0001 & - \\
Initial solids concentration & $\varepsilon_{-} \mathrm{s}_{\text {init }}$ & 0.106 & - \\
Riser diameter & $\mathrm{D}_{\mathrm{t}}$ & 0.09 & $\mathrm{~m}$ \\
Riser height & $\mathrm{h}$ & 10.5 & $\mathrm{~m}$ \\
Overall simulation time & $\mathrm{T}_{-} \mathrm{stop}$ & 20 & $\mathrm{~s}$ \\
Grid size, radial $\times$ axial & $20 \times 150,40 \times 300,60 \times 450$ \\
\hline
\end{tabular}

Additionally, computational results obtained in the last 100 time steps were time and space-averaged on each cross section along the riser of the fluidized bed. The profile of the solid volume fraction was plotted against the available experimental data points. A computer script was used to interpolate the numerical results for specific heights of the riser, where data points from the experiment of Li and Kwauk (1994) were available. Then, the maximum of the deviation of the numerical data points from the corresponding experimental data points was calculated by Eq. (2.30). Further, for each drag model used in this study, an absolute average percentage deviation (AAPD) value, as shown by Eq. (2.31) was calculated.

$$
\operatorname{Err}_{\max }=\operatorname{Max}\left(\left|f_{\text {exp. }}(\lambda)-f_{\text {sim. }}(\lambda)\right|\right)
$$




$$
\text { AAPD }=\operatorname{Err}_{\text {avg. }}(\%)=\frac{100}{N} \times \sum_{\lambda=1}^{N}\left(\left|f_{\text {exp. }}(\lambda)-f_{\text {sim. }}(\lambda)\right|\right) / f_{\text {exp. }}(\lambda)
$$

The quantities $\mathrm{f}_{\text {sim }}$ and $\mathrm{f}_{\text {exp }}$ are numerical and experimental data point values at locations along the riser of the experimental facility, respectively. The parameter $\lambda$ shows the index of the locations and AAPD-9 refers to the label used for the AAPD value calculated using a total of nine (i.e., $\mathrm{N}=9$ ) data points in our initial error calculations. In addition, a polynomial function was fitted to the nine experimental data points in order to create a profile with significantly greater number of points for the error analysis. Thus, the target parameters, such as the correlation values between the numerical and experimental profiles, i.e., $\mathrm{R}^{2}$, and the AAPD, are calculated for 150 points along the riser. Eq. (2.32) shows the expression used for calculation of the $\mathrm{R}^{2}$ value for each constituent and hybrid model.

$$
\left(\mathrm{R}^{2}\right)_{\text {model }}=1-\frac{\sum_{\mathrm{i}=1}^{\mathrm{N}}\left(\mathrm{Y}_{\mathrm{i}, \varepsilon_{s}}-\frac{1}{\mathrm{~N}} \sum_{\mathrm{i}=1}^{\mathrm{N}} \mathrm{Y}_{\mathrm{i}, \varepsilon_{s}}\right)^{2}}{\sum_{\mathrm{i}=1}^{\mathrm{N}}\left(\mathrm{Y}_{\mathrm{i}, \mathcal{\varepsilon}_{S}}-\mathrm{f}_{\mathrm{i}, \varepsilon_{S}}\right)^{2}}
$$

This criterion was used for the overall comparison between the simulation results and the experimental data. In this definition $\mathrm{N}, \mathrm{Y}_{\mathrm{i}, \varepsilon s}$ and $\mathrm{f}_{\mathrm{i}, \varepsilon s}$ indicate the total number of data points, values of $\varepsilon_{S}$ on the polynomial fit, and the values of $\varepsilon_{S}$ on the numerical profile of each drag model, respectively. The improvement to each constituent model was calculated by comparing the Err $\max , \mathrm{R}^{2}$ and AAPD values before and after using the model in our hybrid schemes. Eqs. (2.33) to (2.38) show the expressions used for calculation of these improvements. For brevity, the subscript notations used in Eqs. (2.37) 
and (2.38), i.e., constituent and hybrid, follow the same indexing pattern as used in Eqs. (2.33) to (2.36)

$$
\begin{aligned}
& \left(\operatorname{imp} \cdot \operatorname{Err}_{\text {max }}\right)_{\text {Gidaspow }}=100 \times \frac{\left.\mid\left(\operatorname{Err}_{\max }\right)_{\text {Gidaspow }}-\left(\text { Err }_{\text {max }}\right)_{\text {AGDSM3 }} \mid\right)}{\left(\text { Err }_{\text {max }}\right)_{\text {Gidaspow }}} \\
& \left(\mathrm{imp} . \mathrm{Err}_{\max }\right)_{\text {SY_O'B }}=100 \times \frac{\left.\left|\left(\mathrm{Err}_{\max }\right)_{\text {SY_O'B }}-\left(\mathrm{Err}_{\text {max }}\right)_{\text {AGDSM1 } 1}\right|\right)}{\left(\mathrm{Err}_{\max }\right)_{\text {SY_O'B }}} \\
& \left(\operatorname{imp} \operatorname{Err}_{\text {max }}\right)_{\text {Wen_Yu }}=100 \times \frac{\left.\left|\left(\operatorname{Err}_{\text {max }}\right)_{\text {Wen_Yu }_{1}}-\left(\operatorname{Err}_{\text {max }}\right)_{\text {AGDSM2 }}\right|\right)}{\left(\text { Err }_{\text {max }}\right)_{\text {Wen_Yu }}} \\
& \left(\text { imp.Err }{ }_{\max }\right)_{\mathrm{TGS}}=100 \times \frac{\left.\left|\left(\mathrm{Err}_{\mathrm{max}}\right)_{\mathrm{TGS}}-\left(\mathrm{Err}_{\mathrm{max}}\right)_{\mathrm{AGDSM} 3}\right|\right)}{\left(\mathrm{Err}_{\max }\right)_{\mathrm{TGS}}} \\
& (\text { imp.AAPD })_{\text {constituent }}=100 \times \frac{\mid(\text { AAPD })_{\text {constituent }}-(\text { AAPD })_{\text {hybrid }} \mid}{(\text { AAPD })_{\text {constituent }}} \\
& \left(\mathrm{imp} \_\mathrm{R}^{2}\right)_{\text {constituent }}=100 \times \frac{\left.\left|\left(\mathrm{R}^{2}\right)_{\text {constituent }}-\left(\mathrm{R}^{2}\right)_{\text {hybrid }}\right|\right)}{\left(\mathrm{R}^{2}\right)_{\text {constituent }}}
\end{aligned}
$$

Thresholding constitutes an important feature of all proposed versions of the AGDSM model. Table 2-5 illustrates two extra threshold values, $\varepsilon_{\mathrm{S}}$-THS and $\Theta$-THS, that must be assigned, in addition to the threshold for the Ha parameter. This strategy eliminated the possibility of singularity in Ha calculations in very dilute regions of the domain, where granular temperature was extremely small. Moreover, for cells with extremely small values of granular temperature, no switching operations were executed in the program. 
The optimum values for the variables listed in Table 2-5 are obtained by best practices. Initially, relatively small values were assigned to threshold values, which resulted in a limited variation in numerical simulation. Later, extremely small values were selected for these variables, which resulted in a significant change in results and in some cases, a significant improvement in numerical results were obtained. The Ha-THS parameter was examined in a wide range for all three proposed versions of the drag model in order to find the optimum value.

Table 2-5 Thresholds for solid volume fraction and granular temperature to prevent switching from the standard model to the TGS model.

\begin{tabular}{ccc}
\hline Assignment & & Condition (thresholding) \\
\hline $\mathrm{Ha}=0$ & for & $\varepsilon_{\mathrm{s}}<\varepsilon_{\mathrm{S}}-\mathrm{THS}$ \\
$\mathrm{Ha}=0$ & for & Theta_m $\leq \Theta$-THS \\
\hline
\end{tabular}

\subsection{Results and Discussion}

Figure 2-2 illustrates the results obtained with the TGS drag model and the other existing drag models on three different computational grids (See Figure 2-1). Figure 2-2(a) illustrates the grid independency study with the TGS drag model where the computational grid $(40 \times 300)$ was found to be optimum. It was observed that when the existing drag models or the TGS model were used alone, the solid volume fraction profile in the riser showed significant deviation from the experimental data given by Li and Kwauk(1994). Figure 2-2(d) shows that all models produce very similar results with the increase of the computational grid size, and these results are in good agreement with the simulation 
results obtained by Hong et al. (2012), where they used the Gidaspow drag model in their simulation of the same flow.

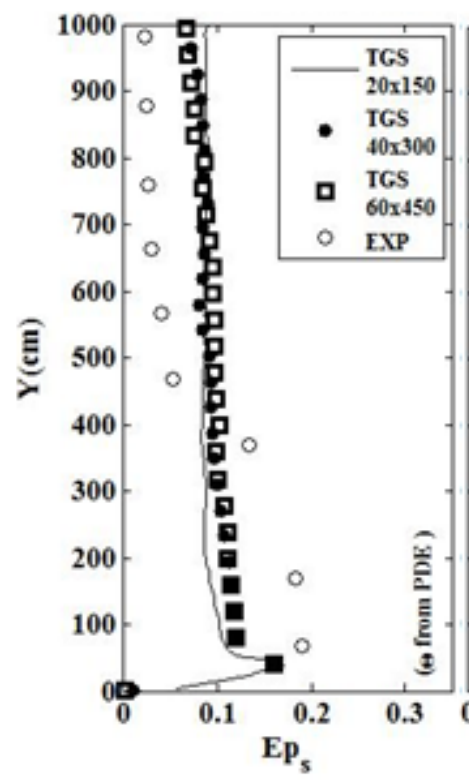

(a)

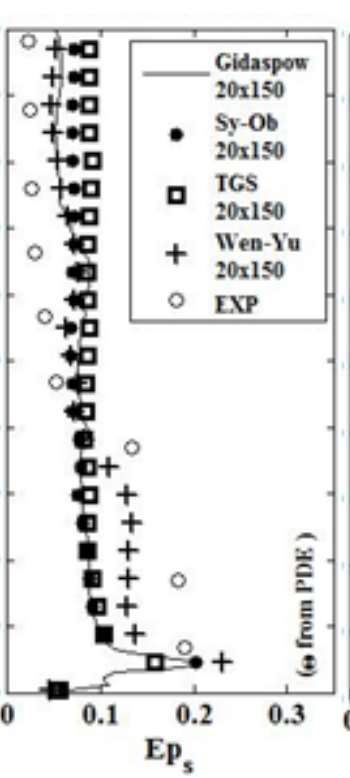

(b)

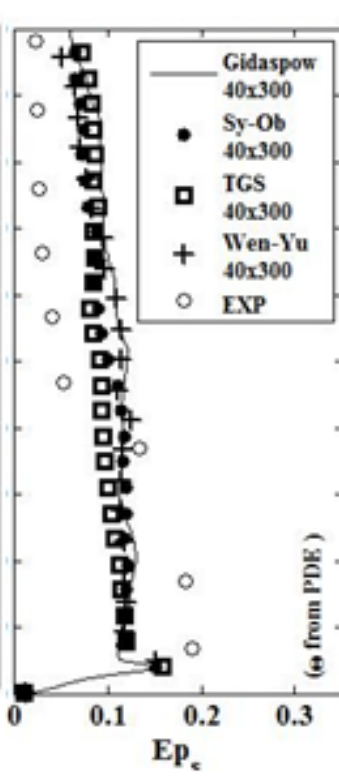

(c)

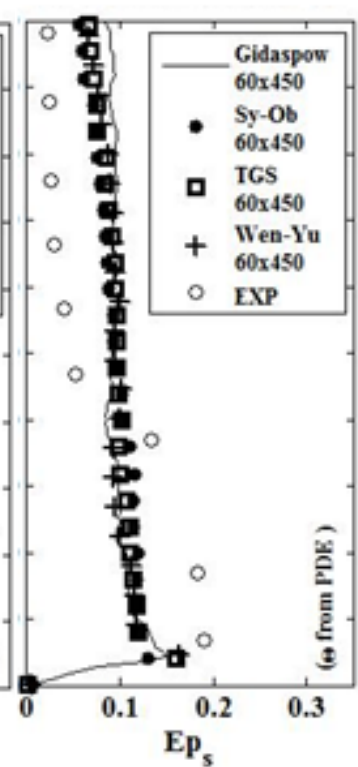

(d)

Figure 2-2 Profile of solid volume fraction along the riser of the fluidized bed, MFIXTFM results with TGS and existing drag models on three computational grid sizes.

Table 2-6 shows the maximum and relative errors in the simulation results for the computational grid size of $20 \times 150$. The maximum and minimum values for errors were observed in the cases with the TGS and Wen-Yu correlations, respectively. The results are comparable to the simulation results obtained by Hong et al. (2012) for the same geometry and boundary conditions. Further, in order to evaluate the sensitivity of the AGDSM versions to the threshold values, $\varepsilon_{\mathrm{S}}$-THS and $\Theta$-THS, these parameters were varied according to the modes given in Table 2-7. 
Table 2-6 Error in numerical simulation compared to available experimental data of Li and Kwauk (1998) for computational grid size of $20 \times 150$.

\begin{tabular}{lcc}
\hline Simulation Case & Max. error & Avg. error (\%) \\
\hline Hong et al. (date) & 0.0773 & 166 \\
Syam-O’Brien & 0.0954 & 106 \\
Gidaspow & 0.0959 & 93 \\
Wen-Yu & 0.0545 & 77 \\
TGS & 0.0918 & 143 \\
\hline
\end{tabular}

Table 2-7 Variation of the thresholds in the AGDSM models

\begin{tabular}{cccc}
\hline & Mode-1 & \multicolumn{2}{c}{ Mode-2 } \\
\hline Parameter & Threshold value & Parameter & Threshold value \\
\hline$\varepsilon_{\mathrm{S}}$-THS & 0.02 & $\varepsilon_{\mathrm{S}}$-THS & $1 \times 10^{-3}$ \\
$\Theta$-THS & $0.0008\left(\mathrm{~cm}^{2} / \mathrm{s}^{2}\right)$ & $\Theta$-THS & $1 \times 10^{-16}\left(\mathrm{~cm}^{2} / \mathrm{s}^{2}\right)$ \\
Ha-THS & {$\left[1 \times 10^{-5}-1\right]$} & Ha-THS & {$\left[1 \times 10^{-10}-0.1\right]$} \\
\hline
\end{tabular}

Figure 2-3 shows the effect of switching operations on simulation results under the constraints of the first mode, as explained in Table 2-7. In this figure, the immediate observation from Figure 2-3(a-b) is that, for various values of the Ha-THS parameter and the fixed values of $\varepsilon_{\mathrm{S}}$-THS and $\Theta$-THS, a significant alteration in the solid volume fraction profile occurred. However, identical results were obtained for the Ha-THS parameter in a broad range of variation (e.g., $\left[1 \times 10^{-5}\right.$ to 1$\left.]\right)$. In addition, improvement of the numerical results, in terms of deviation from the experimental data, was limited to only small portions of the computational domain in high and low sections of the riser. Further, Figure 2-3(c-f) show that the effect of filtration by the model constraints is more pronounced for AGDSM2 and AGDSM3 versions of the proposed model. According to 
Figure 2-3(c-d) and (e-f) for the AGDSM2 and AGDSM3 models, respectively, no alteration of the results was observed in a significantly wider range of the Ha-THS parameter (e.g., $\left[1 \times 10^{-5}\right.$ to $\left.1 \times 10^{4}\right]$ ). In fact, the extremely conservative nature of the filtration procedure, Table 2-5, accounts for the unnecessary elimination of switching operations in the regions where relatively large values of Ha were detected. This observation helps to understand that although the onset of the changes in the simulation results of the AGDSM1 model, occurred at large values of the threshold (Ha-THS = 0.05), the loss of the sensitivity to smaller values of the Ha-THS in our modeling could be overcome by significant reduction of the values for the $\Theta$-THS and $\varepsilon_{\mathrm{S}}$-THS parameters (Mode $=2$ in Table 2-7). Here we refer to this treatment as simulation with relaxed constrains and we have shown that this treatment was effective for all versions of the proposed drag model. 


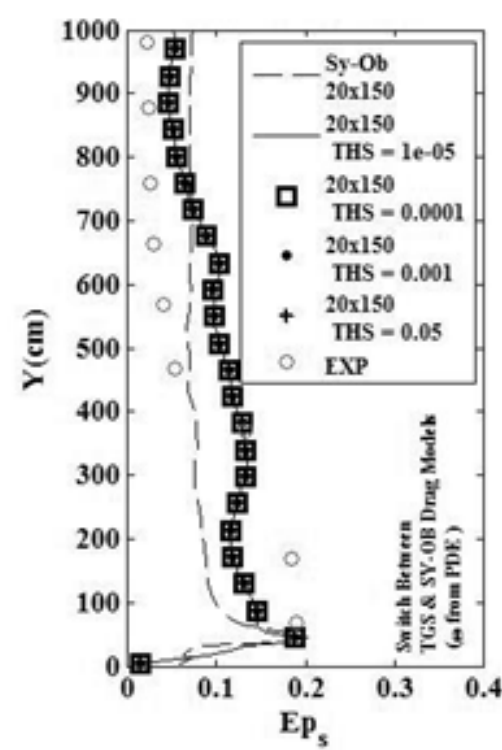

(a)

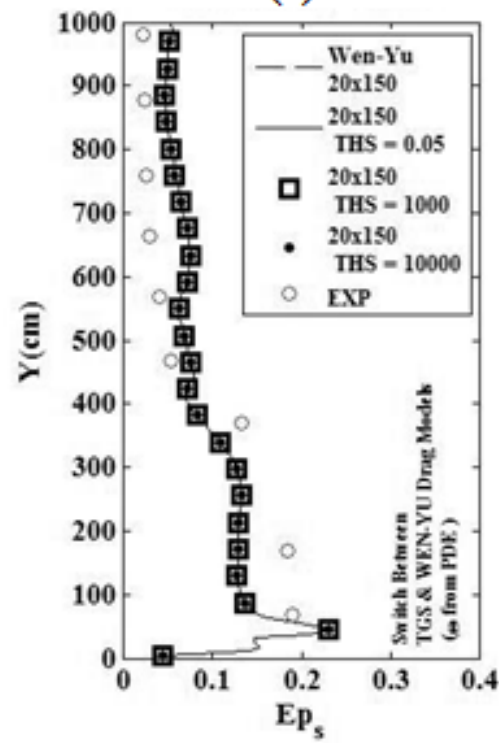

(d)

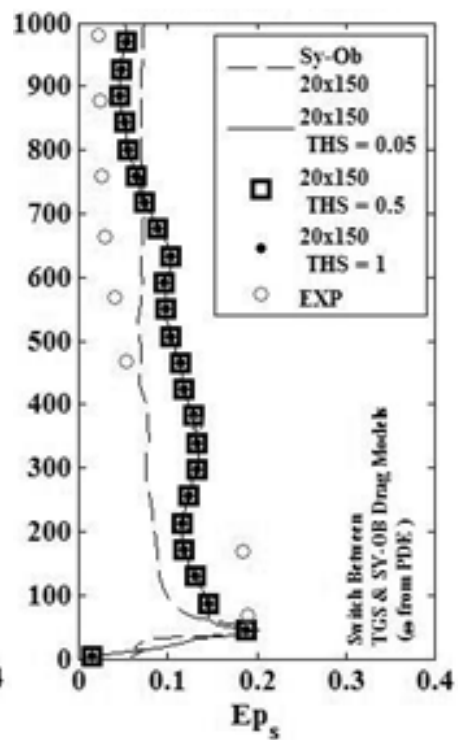

(b)

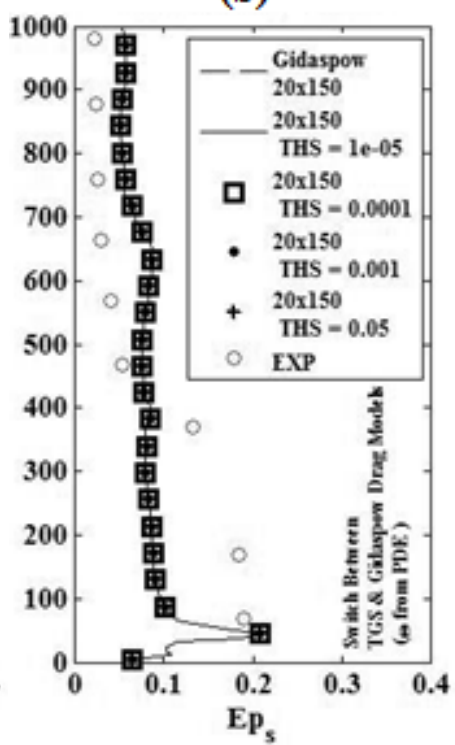

(e)

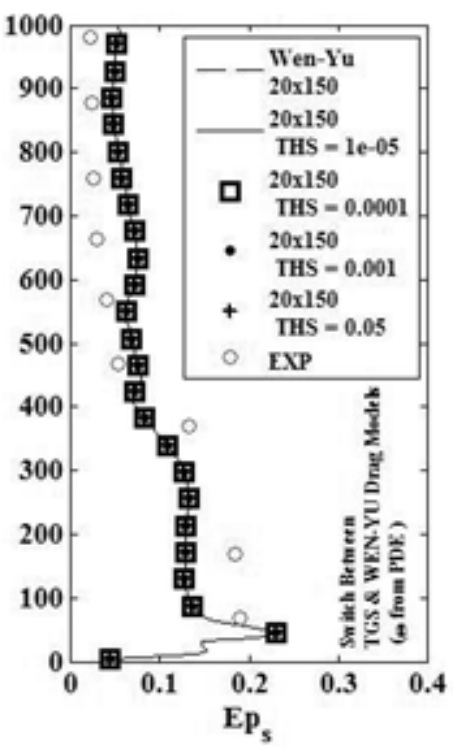

(c)

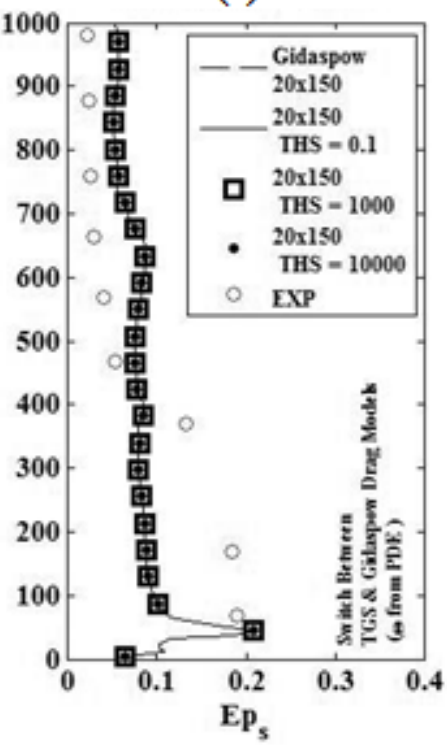

(f)

Figure 2-3 Switching effects in the three versions of the AGDSM model on time and area-averaged profile of the solid volume fraction along the riser against variation of the Ha-THS 


\subsubsection{Simulation Results with Relaxed Constraints}

Simulation results with relaxed constrains, introduced as mode 2 in Table 2-7, are displayed in Figure 2-4 for the AGDSM1, AGDSM2 and AGDSM3 versions. For the AGDSM1 model, Figure 2-4(a) shows an identical $\varepsilon_{\mathrm{S}}$ profile for $0.1 \leq$ Ha-THS $<0.0001$. According to Figure 2-4(b-c), the onset of the ongoing changes occurred at Ha-THS = $1 \times 10^{-4}$ and significant improvements were observed for Ha-THS $=1 \times 10^{-5}$, Ha-THS $=$ $1 \times 10^{-9}$, and Ha-THS $=1 \times 10^{-10}$. However, remarkably large deviations from the experimental profile are observed for $1 \times 10^{-6} \leq$ Ha-THS $\leq 1 \times 10^{-8}$, where the ASDSM1 version almost regenerated the $\varepsilon_{\mathrm{s}}$ profile of the TGS model at Ha-THS $=1 \times 10^{-6}$. The AGDSM2 model, as shown in Figure 2-4(d), reproduced the original $\varepsilon_{s}$ profile of the Wen-Yu model for $0.5 \leq$ Ha-THS $<0.0001$. Here, unlike the AGDSM1 model, the onset of the changes occurred at Ha-THS $=1 \times 10^{-4}$ and we observed a dynamic variation of results after this threshold value. Here, consecutive increase and decrease of the deviations from the experimental results were observed with the reduction of the HaTHS. In addition, the AGDSM2 version almost regenerated the $\varepsilon_{s}$ profile of the TGS model at two threshold values, e.g., Ha-THS $=1 \times 10^{-6}$ and Ha-THS $=1 \times 10^{-8}$. However, significant improvements in the agreement with the experimental values were obtained for the Ha-THS $=1 \times 10^{-9}$ and Ha-THS $=1 \times 10^{-10}$. The AGDSM3 model, as shown in Figure 2-4(g), reproduced the original $\varepsilon_{s}$ profile of the Gidaspow model for $0.1 \leq$ Ha-THS $<0.0001$. Similar to the AGDSM2 version, we observed a dynamic variation of results after the Ha-THS $=1 \times 10^{-4}$. Further, Figure 2-4(g-i) shows consecutive increase and decrease of the deviations of the numerical results from the experimental results and the 
model almost reproduced the original $\varepsilon_{s}$ profile of the TGS model for the intermediate value of the threshold (e.g., Ha-THS $=1 \times 10^{-6}$ ). However, significantly better agreements with the experimental values were obtained for the Ha-THS $=1 \times 10^{-5}$ and Ha-THS $=$ $1 \times 10^{-7}$. Further, to improve our error analysis, we plotted the profiles of the maximum and relative errors as displayed in Figure 2-5(a) and (b), respectively. Here, we noticed a similarity between the profiles of the maximum and the relative errors for each version of the proposed drag model. Figure 2-5(a) shows that the maximum improvements for the AGDSM1, AGDSM2, and AGDSM3 happened at Ha-THS $=1 \times 10^{-10}$, Ha-THS $=1 \times 10^{-10}$, and Ha-THS $=1 \times 10^{-10}$, respectively. In addition, Figure 2-5(a-b) shows that no consistent trend of reduction or escalation of error against values of the Ha-THS parameter could be traced for all proposed versions of the AGDSM model. Moreover, the models did not develop similar patterns of variation for the profiles of Errmax and Erravg errors. Our observation is that perturbations on the error profiles start at Ha-THS $=1 \times 10^{-}$ ${ }^{3}$ for all AGDSM versions and all models approached the Err $\max$ value of the TGS model at Ha-THS $=1 \times 10^{-6}$ (Figure 2-5-a).

Another observation from Figure 2-5(a-b) is that the level of error associated with the AGDSM3 model is significantly lower than the other two models for $1 \times 10^{-8} \leq$ Ha-THS $<$ $1 \times 10^{-4}$. In other words, switching between the TGS and the Gidaspow drag models could optimally serve the objective of the present research, which is to improve the performance of the existing standard drag modes through a combination with a DNSbased drag model. 


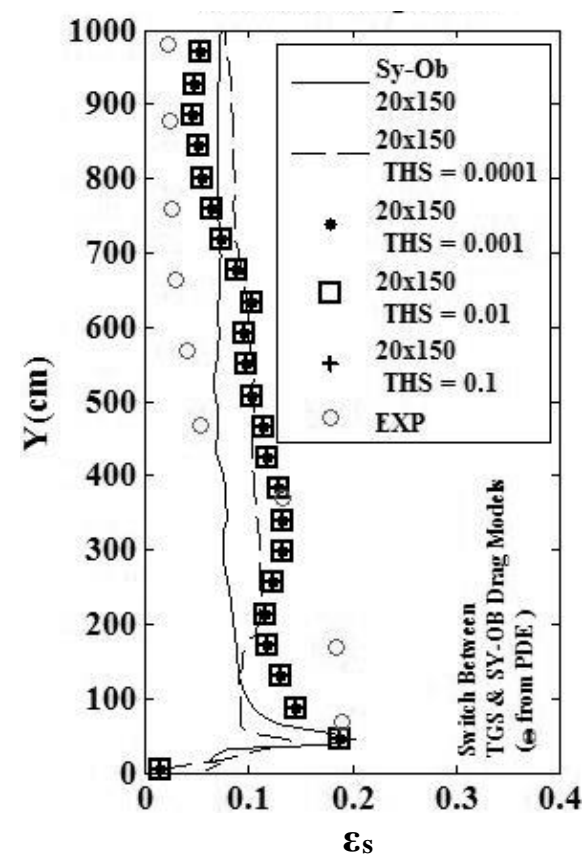

(a)

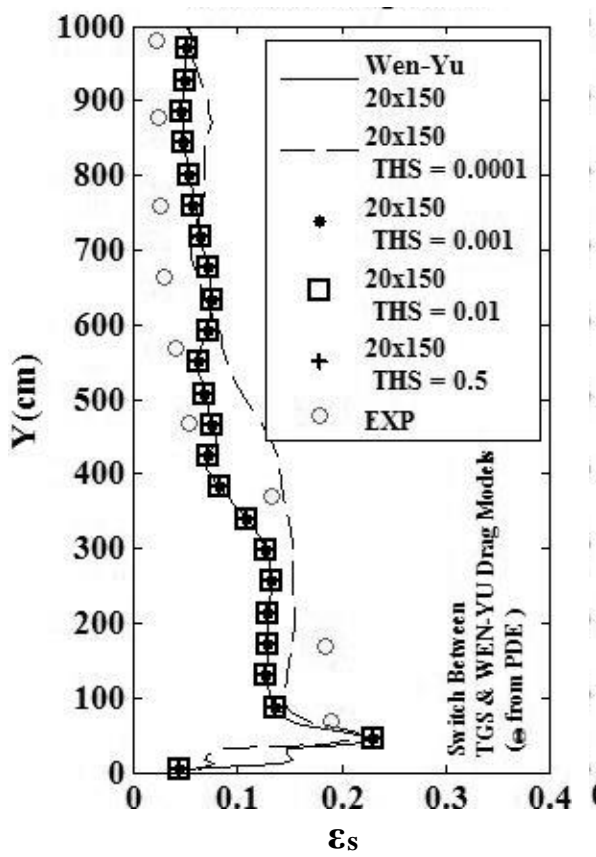

(d)

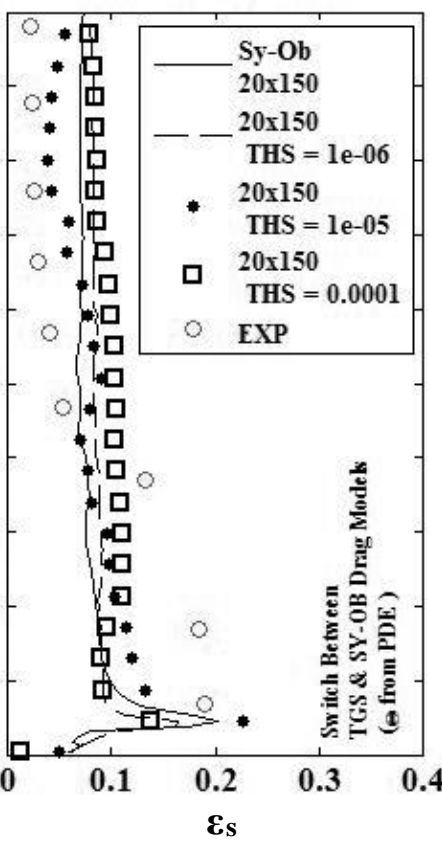

(b)

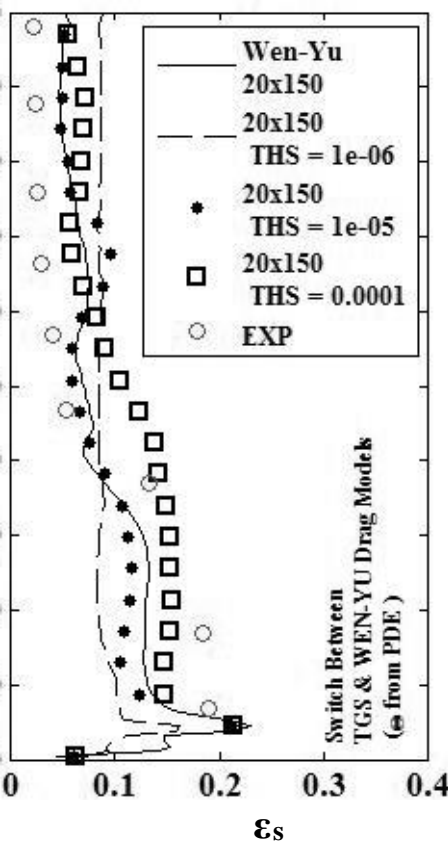

(e)

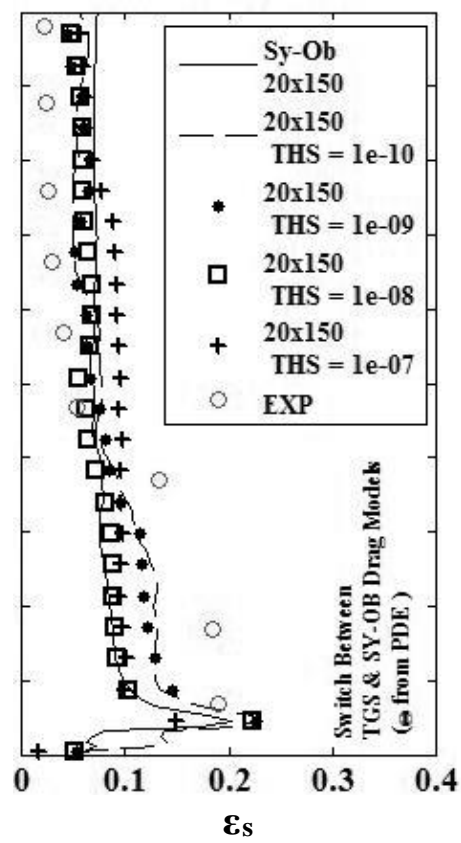

(c)

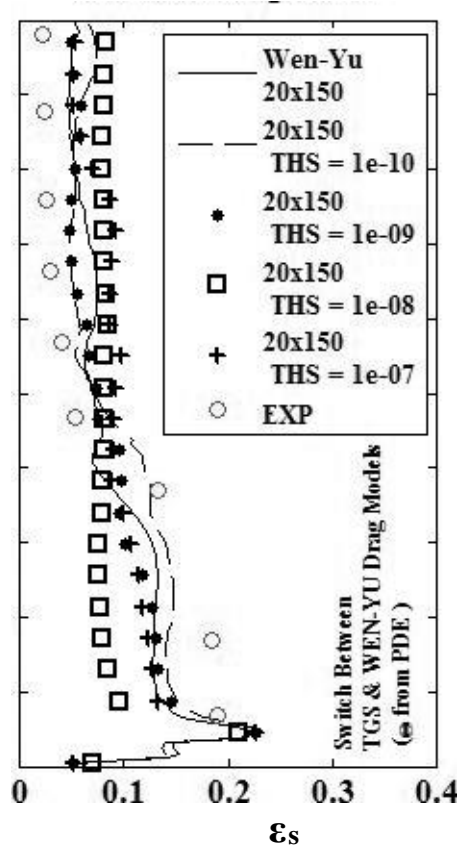

(f) 


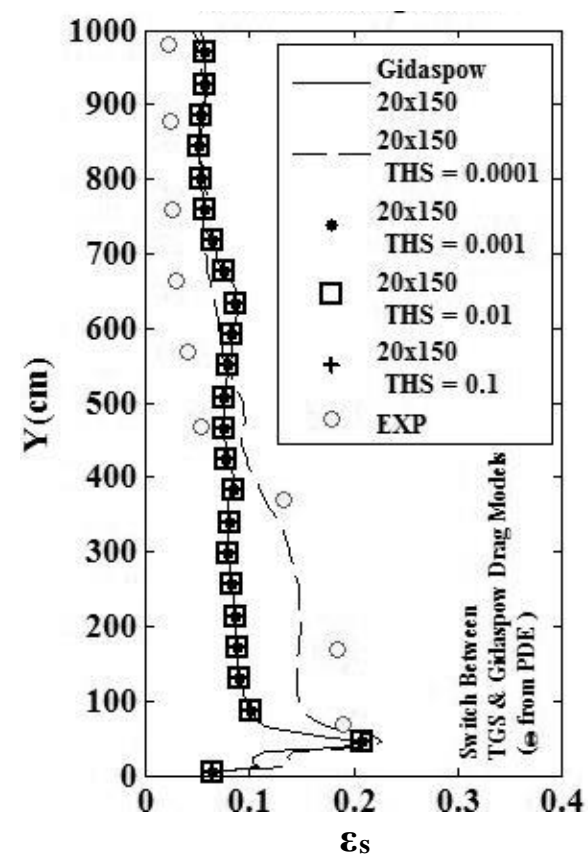

(g)

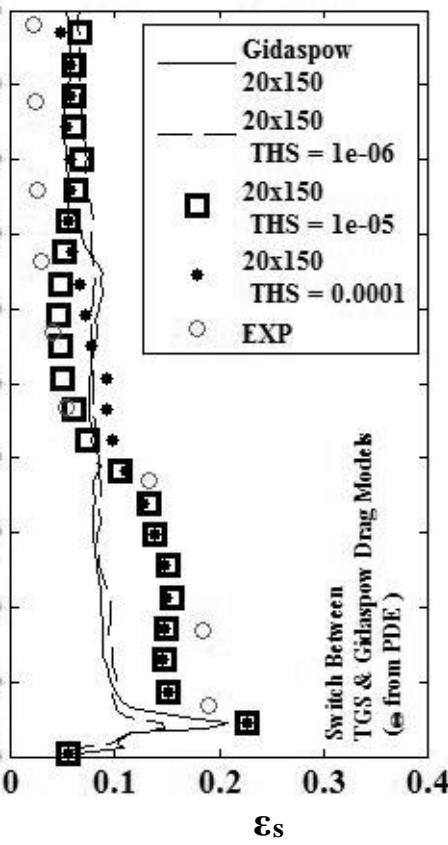

(h)

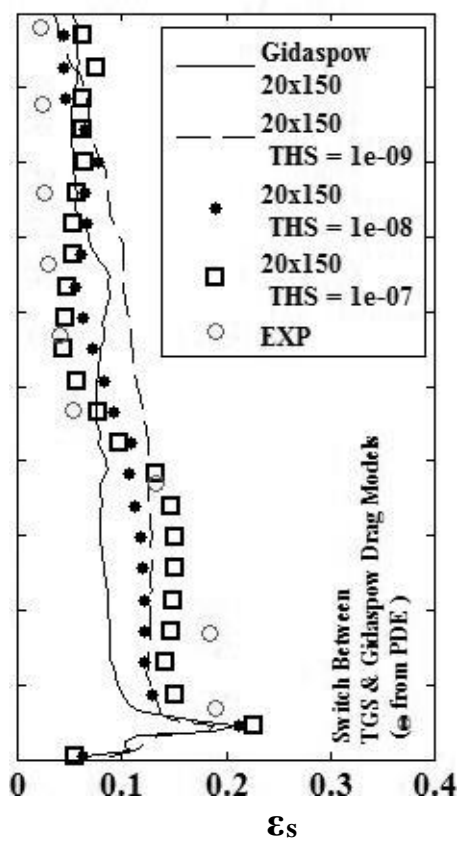

(i)

Figure 2-4 Profile of solid volume fraction $\left(\varepsilon_{\mathrm{s}}\right)$ from MFIX_TFM simulations for various values of Ha-THS; (a to c) represent AGDSM1, (d to f) represent the AGDSM2, and (g to i) represent the AGDSM3.

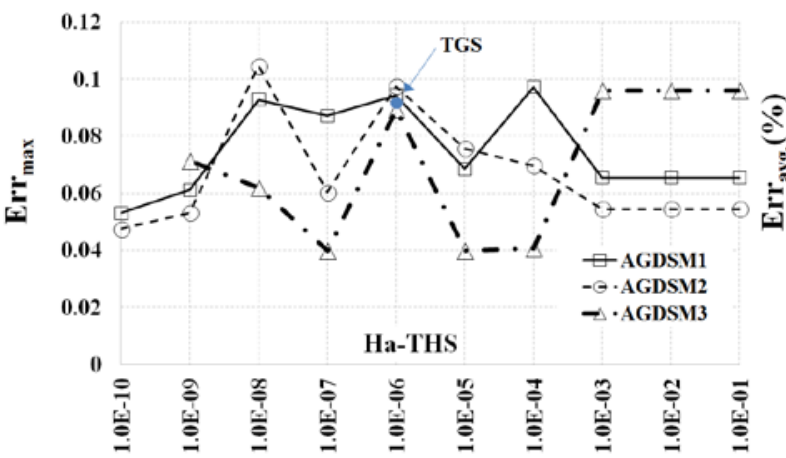

(a)

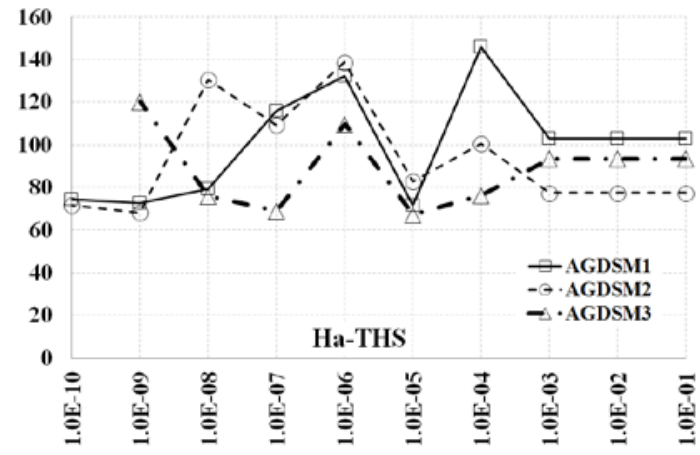

(b)

Figure 2-5 Error in TFM simulation for three versions of the proposed drag model, maximum of error on left and averaged relative error on right.

Figure 2-6 illustrates a thorough comparison between the best results obtained by our proposed hybrid models and their corresponding constituent standard drag model. We 
first demonstrated in Figure 2-6(a-c) that all hybrid models significantly outperformed their corresponding constituent standard drag model. According to Figure 2-6(c), the most effective switch operations were performed by the AGDSM3 model. Later, we demonstrated that all the proposed hybrid models compared closely to each other and agreed decently with the experimental data values. Perhaps the best agreement was obtained by the AGDSM3 model and this statement needs further quantification of all error criteria mentioned before.

The error values shown in the Table 2-8 demonstrate that the smallest values of 9-point maximum error and AAPD values calculated using Eqs. (2.30) and (2.31) were obtained for the AGDSM3 model under optimal conditions. However, in our analysis with a 4th order polynomial fit with a high fit quality of $\mathrm{R}^{2} \approx 0.98$ to the experimental data points, as shown in Figure 2-7, slightly smaller values of AAPD were observed for the AGDSM2 model version. In a similar fashion, the largest 9-point $\mathrm{R}^{2}$ values were obtained for the AGDSM3 model version, while this model version possessed the second largest $\mathrm{R}^{2}$ values after the AGDSM2 model version for the polynomial fit. For brevity, the 9-point $\mathrm{R}^{2}$ values are not shown in the Table 2-8. 


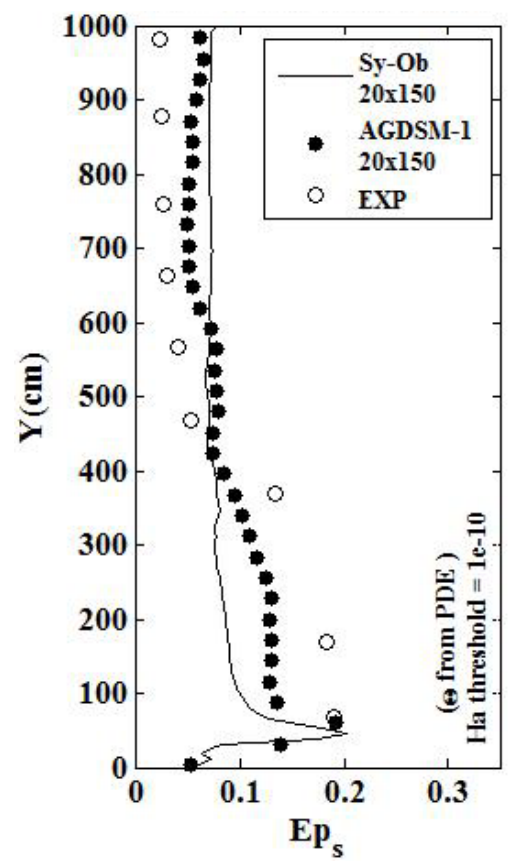

(a)

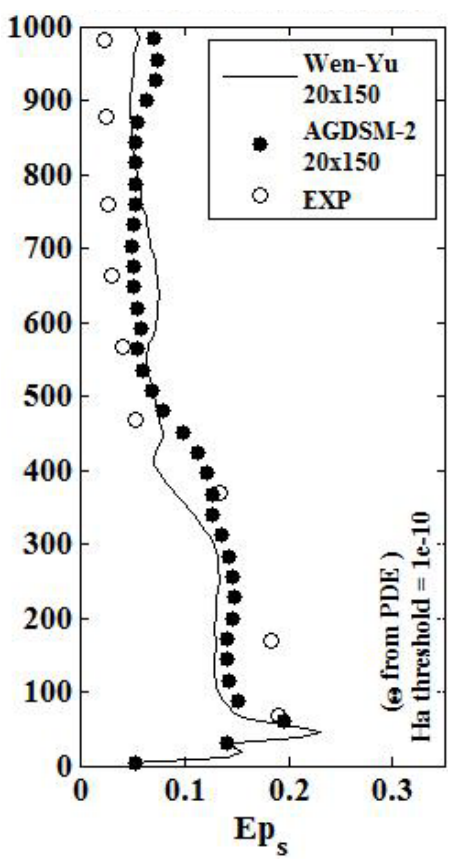

(b)

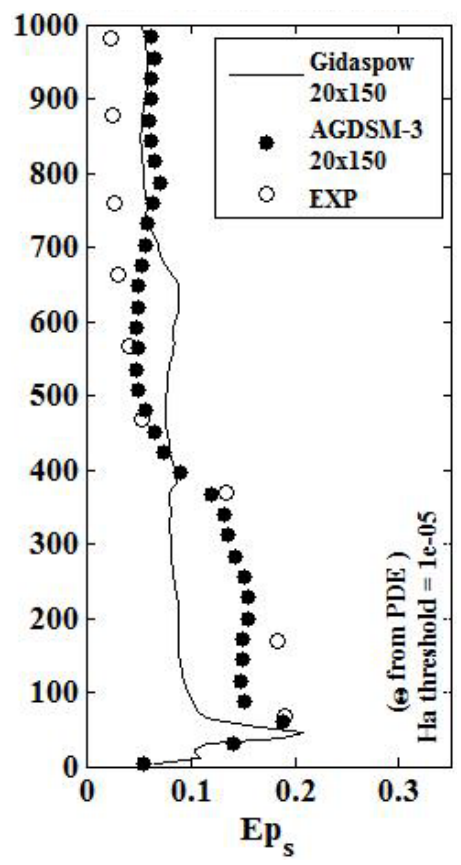

(c)

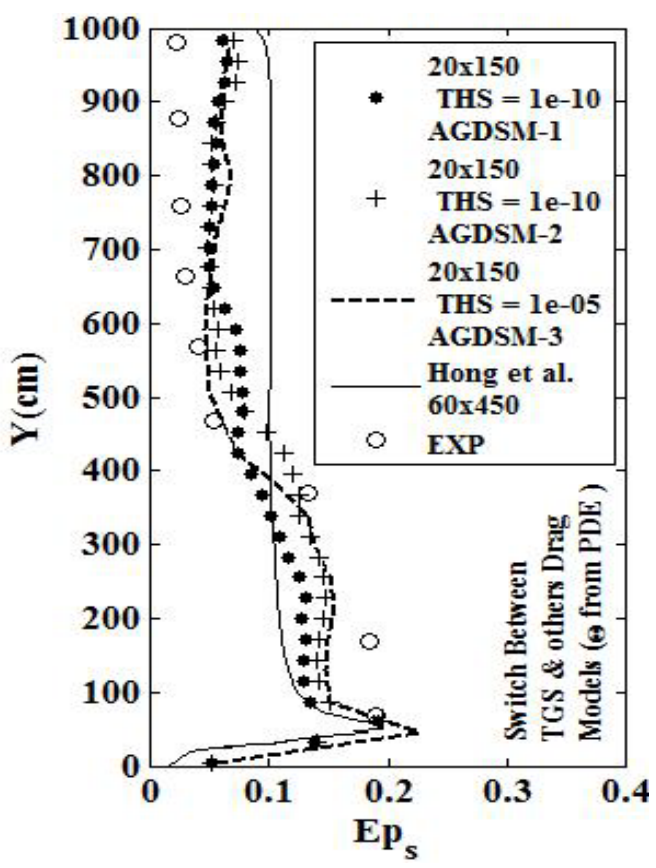

(d)

Figure 2-6 Optimal MFIX-TFM simulations by versions of the AGDSM model, (a) to (c) are versions of the AGDSM model versus corresponding standard models, (d): best result from AGDSM1-3 versions and the profile of Hong et al. (2012) 


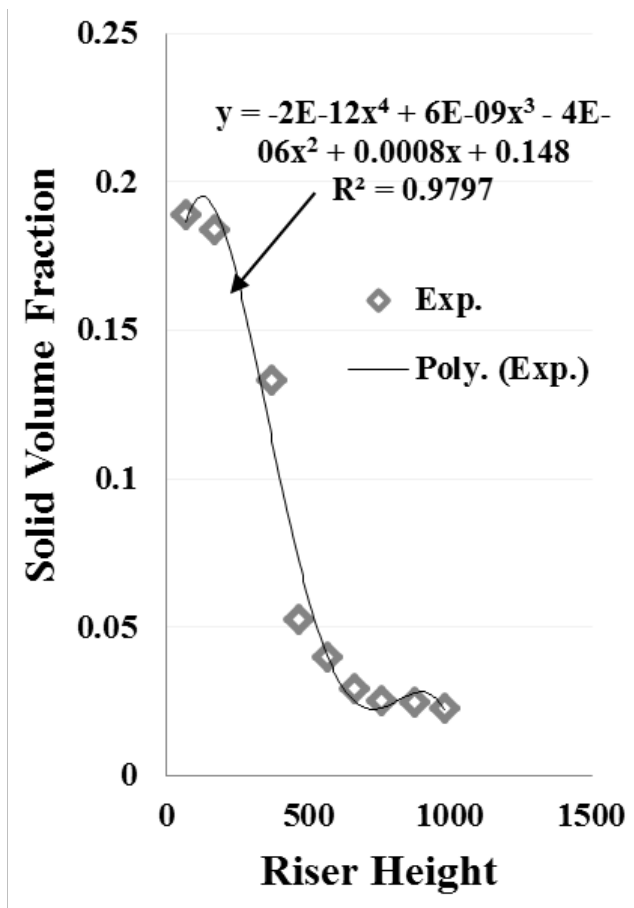

Figure 2-7 Fourth-order fit to the experimental data of Li and Kwauk (1994)

Table 2-8 also shows the results of our error and $\mathrm{R}^{2}$-improvement analysis. Accordingly, all hybrid models used in this study demonstrated high correlation values $\left(\mathrm{R}^{2} \geq 0.95\right)$ with the experimental profile. In the case of standard drag models used in this study, the best improvements in terms of maximum-error (\%58.4) and $\mathrm{R}^{2}$-value (\%30.1) were obtained for the Gidaspow drag model. In addition, we were able to improve the results of the Syam-O'Brien drag model in terms of AAPD criterion for the largest improvement value of 32\%. This accomplishment is significant since this improvement was obtained without activating the cluster-related correction parts of the Syam-O'Brien drag subroutine in the MFIX. 
In addition, it is possible to make qualitative comparisons between profiles of solid volume fraction in Figure 2-6(d) and quantitative comparison between the error and $\mathrm{R}^{2}$ values on the first and the last three rows of the Table 2-8. These comparisons reveal the improved performance of all proposed AGDSM model versions in optimal conditions over the results reported by Hong et al. (2012) where the Gidaspow model was used on a significantly finer computational grid $(60 \times 450)$. The purpose of this comparison is to show that significantly better computational results could be obtained by only combining the non-structured based drag models with the particle resolved-DNS TGS drag model, and without a need for refining the computational grid size.

Table 2-8 Error calculations and best improvements for different versions of the AGDSM model

\begin{tabular}{|c|c|c|c|c|c|c|c|}
\hline Simulation case & Err. max & $\begin{array}{l}\text { AAPD-9 } 1 \\
\text { (\%) }\end{array}$ & $\begin{array}{c}\text { AAPD-fit }^{2} \\
(\%)\end{array}$ & $\mathrm{R}^{2}$ & $\begin{array}{l}\text { imp.Err }_{\text {max }} \\
(\%)\end{array}$ & $\begin{array}{c}\text { imp_AAPD } \\
(\%)\end{array}$ & $\begin{array}{c}\text { imp_R } R^{2} \\
(\%)\end{array}$ \\
\hline $\begin{array}{l}\text { Hong et al. } \\
\text { (2012) }\end{array}$ & 0.0773 & 166.1 & 138.5 & 0.557 & - & - & - \\
\hline Syam-O’Brien & 0.0954 & 106.2 & 100.7 & 0.789 & 44.5 & 32.0 & 23.2 \\
\hline Gidaspow & 0.0959 & 93.5 & 87.9 & 0.735 & 58.4 & 26.4 & 30.1 \\
\hline Wen-Yu & 0.0545 & 77.4 & 71.0 & 0.956 & 12.9 & 10.5 & 1.12 \\
\hline TGS & 0.0918 & 143.1 & 132.4 & 0.410 & 56.5 & 51.14 & 133.3 \\
\hline AGDSM $1^{3}$ & 0.0531 & 74.5 & 68.4 & 0.973 & - & - & - \\
\hline AGDSM $2^{4}$ & 0.0474 & 71.6 & 63.6 & 0.966 & - & - & - \\
\hline AGDSM3 $^{5}$ & 0.0399 & 67.2 & 64.7 & 0.956 & - & - & - \\
\hline
\end{tabular}

1: 9-point absolute average percent deviation

2: absolute average percent deviation using the $4^{\text {th }}$ order polynomial fit

$3:$ Ha-THS $=1 \times 10^{-10}$

$4:$ Ha-THS $=1 \times 10^{-10}$

$5:$ Ha-THS $=1 \times 10^{-5}$ 


\subsubsection{Switching Frequency and Improvement in Hybrid Model}

Results shown earlier in Figure 2-5 indicated an unrecognizable relation between the variation of error and the value of the Ha-THS parameter. The switching mechanism between constituent drag models, as explained earlier in Table 2-1, was controlled by two parameters, Ha and Ha-THS. Therefore, the results listed in Table 8 encouraged us to explore the relation between the improvements for the proposed versions of the AGDSM model and the switching frequencies occurred in the simulations. For this purpose, we first calculated the number of the computational cells which met the criteria of switching for all proposed hybrid drag versions. In this method, the frequency of switching operations for AGDSM3 under the best condition (i.e., Ha-THS $=1 \times 10^{-5}$ ) was the number of cells that met the condition of $\mathrm{Ha}>1 \times 10^{-5}$ and similar definition was used for the AGDSM2 and AGDSM3 versions with Ha- THS $=1 \times 10^{-10}$. For the constituent models, we calculated the number of the computational cells which could potentially meet the switching criterion of $\mathrm{Ha}>1 \times 10^{-10}$. The importance of investigation about these criteria was to evaluate the potential of further modification to both the constituent and hybrid drag models based on the relative strangeness of cohesive forces, i.e., cluster formation. Figure 2-8 shows the potential of further modification to all drag models used in this study. These results show significant differences between the constituent models, presented in Figure 2-8(a), and the proposed hybrid model versions, presented in Figure 2-8 (b). Surprisingly, there is a direct relation between the improvement to the AAPD values listed in the Table 2-8 and the potential modifications shown in Figure 2-8(a). In other words, the TGS model with the highest AAPD values, i.e., \%132.4, required the most number of switching operations, i.e., 2774, and possessed the highest level of 
overall modification (imp-AAPD), i.e., \% 51.14. In a similar fashion, the Wen-Yu model with the lowest AAPD, required the smallest number of operations and possessed the lowest level of AAPD improvements. Based on these observations, significant modifications shown in Figure 2-6 and Table 2-8 can be explained by the significant changes which occurred to the constituent models through switching operations between the standard models and the TGS model. However, the relationship between the large number of switching operations required for the Wen-Yu model and the level of modification to this model, as described by Table 2-8, necessitates more attention to be given to the effectiveness of switching operations for different models. Therefore, further research is necessary to investigate about the relationship between cohesive index and field variables, such as granular temperature of the solid phase and drag force coefficients. (See Fig. 2-A-1)

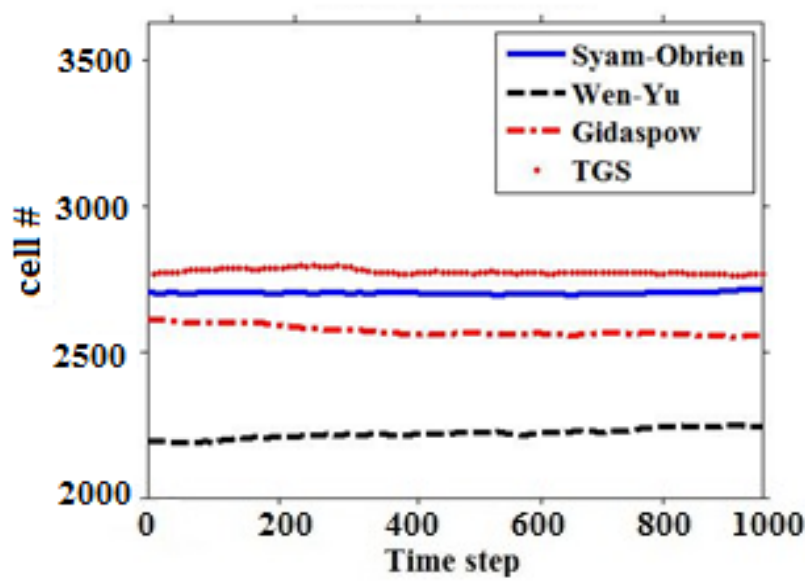

(a)

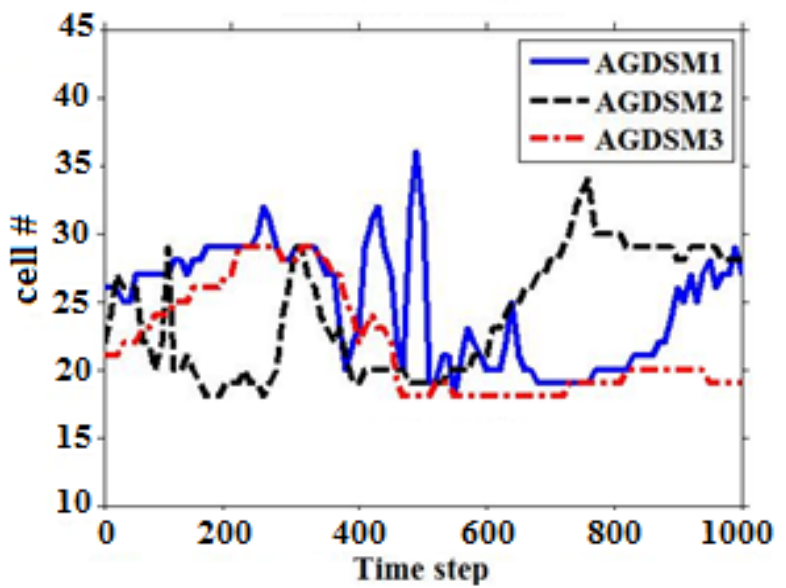

(b)

Figure 2-8 Frequency of switch operations. (a) constituent drag models, (b) proposed AGDSM model versions in optimal cases 


\subsection{Conclusion}

In this paper, we have demonstrated that TFM simulations of the air-FCC flow could not produce accurate results using the original form of three existing standard drag models in the MFIX package (i.e., Syam-O'Brien, Gidaspow and Wen-Yu models) and the TGS model, as a particle resolved DNS drag model. On the other hand, combining a standard drag model with the TGS model, under optimized conditions and based on a switching mechanism, proved to be a useful method to significantly improve the accuracy of the numerical results. In this approach, the switching mechanism proved to be exceptionally sensitive to the variation of the threshold values of the cohesive index, Ha-THS. A direct relationship between the error improvements and the frequency of switch operations in the proposed models is recognized. Consequently, models with more numerical error demanded more switching operations and experienced more modifications. However, improvements to the constituent models must be considered together with the quality of the switch operations. This can lead to establishment of an accurate relationship between the drag force and the cohesive index, hence a more comprehensive drag force model wherein the cohesive index is explicitly incorporated into its formulation. In conclusion, the proposed approach was observed to be successful for all the three drag model versions under optimal conditions, where a maximum of almost $60 \%$ improvement in accuracy of simulation results was obtained for the Gidaspow model. Therefore, a direct or indirect implementation of particle clustering and ensuing modifications in the TFM approach is necessary to be practiced for gas-solid flows under the influence of cohesive interparticle forces. 


\subsection{Appendix A.}

Relative equation of motion of particles

For particle i:

$$
m_{i} \frac{d^{2} \vec{x}_{i}}{{d t^{2}}^{2}}=\vec{F}_{c, i}+\vec{F}_{v d w, i}+\vec{F}_{d r a g, i}-V_{i} \nabla p+m_{i} \vec{g}
$$

For particle j:

$$
\begin{gathered}
m_{j} \frac{d^{2} \vec{x}_{j}}{d t^{2}}=\vec{F}_{c, j}+\vec{F}_{v d w, j}+\vec{F}_{d r a g, j}-V_{j} \nabla p+m_{j} \vec{g} \\
\vec{F}_{c, i}=\vec{F}_{c, j}=0
\end{gathered}
$$

and

$$
\begin{aligned}
\overrightarrow{\mathrm{F}}_{\text {drag,i }} & =\overrightarrow{\mathrm{F}}_{\text {drag,j }} \\
\mathrm{V}_{\mathrm{i}} \nabla \mathrm{p} & =\mathrm{V}_{\mathrm{j}} \nabla \mathrm{p} \\
\mathrm{m}_{\mathrm{i}} \overrightarrow{\mathrm{g}} & =\mathrm{m}_{\mathrm{i}} \overrightarrow{\mathrm{g}} \\
\overrightarrow{\mathrm{F}}_{\mathrm{vdw}, \mathrm{i}} & =-\overrightarrow{\mathrm{F}}_{\mathrm{vdw}, \mathrm{j}}
\end{aligned}
$$

Fig. 2-A-1 shows the schematic of the particles with small separation distance from each other under the effect of the van der Waals force, the gas-particle drag force, the pressure gradient in the fluid, and the gravitational force. Subtraction of Eq. (A.2) from Eq. (A.1) results in cancelation of some of the terms and the following expression was obtained

$$
\frac{\mathrm{d}^{2}\left(\overrightarrow{\mathrm{X}}_{\mathrm{i}}-\overrightarrow{\mathrm{X}}_{\mathrm{j}}\right)}{\mathrm{dt}^{2}}=\left(\frac{1}{\mathrm{~m}_{\mathrm{i}}} \overrightarrow{\mathrm{F}}_{\mathrm{vdw}, \mathrm{i}}-\frac{1}{\mathrm{~m}_{\mathrm{j}}} \overrightarrow{\mathrm{F}}_{\mathrm{vdw}, \mathrm{j}}\right) .
$$


For the cohesive inter-particle force, we adopt the Hamaker expression [60] for two spheres, given by the following equation

$$
\left|\vec{F}_{v d w, i j}(d)\right|=\frac{A}{3} \frac{2 R_{i} R_{j}\left(d+R_{i}+R_{j}\right)}{\left[d\left(d+2 R_{i}+2 R_{j}\right)\right]^{2}} \times\left[\frac{d\left(d+2 R_{i}+2 R_{j}\right)}{\left(d+2 R_{i}+2 R_{j}\right)^{2}-\left(R_{i}-R_{j}\right)^{2}}-1\right]^{2} .
$$

In this equation, $\mathrm{A}$ is the Hamaker constant, $\mathrm{R}$ is the radius of the particle and $\mathrm{d}$ is the surface-to-surface distance between the two particles i and $\mathrm{j}$. This expression simplifies to $\overrightarrow{\mathrm{F}}_{\mathrm{vdw}, \mathrm{ij}}(\mathrm{d})=\left(\mathrm{AR} / 6 \mathrm{~d}_{\mathrm{ij}}{ }^{2}\right) \overrightarrow{\mathrm{n}}_{\mathrm{ij}}$ for two spheres of the same diameter. Later, by placing the frame of reference on the particle $\mathrm{j}$, and replacing the dij with the distance d, in Fig. 2-A1, Eq. (A.8) can be reformatted as

$$
\left(\frac{m_{i} m_{j}}{m_{i}+m_{j}}\right) \frac{d \vec{V}_{\text {rel. }}{ }^{(i)}}{d t}+\frac{A R}{6 d^{2}}=0 .
$$

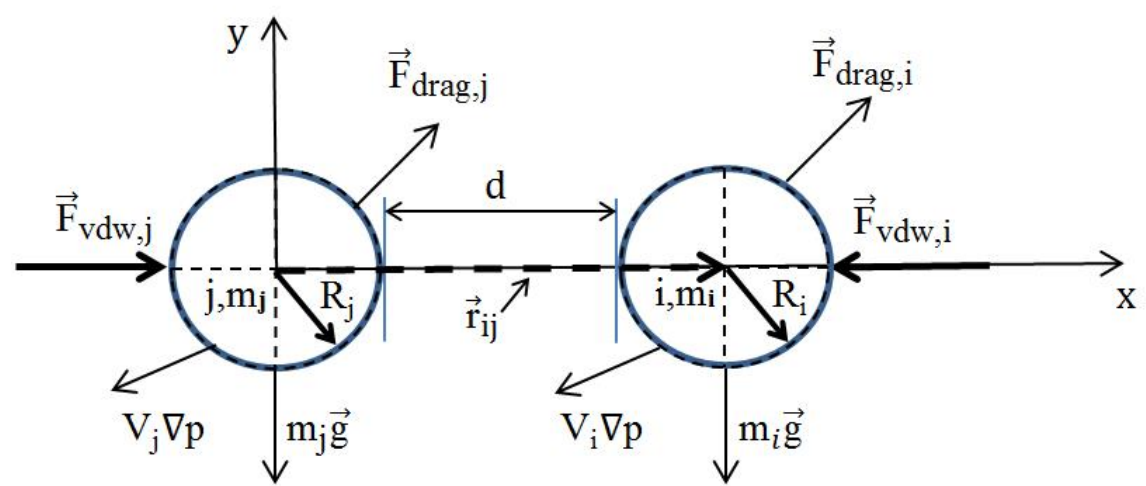

Fig. 2-A-1. Representation of the van der Waals force, the gas-particle drag force, the force due to pressure gradient in the fluid, and the gravitational force acting on two spherical particles with equal radius. 


\subsection{References}

A.T. Andrews IV, P.N. Loezos, S. Sundaresan, Coarse-grid simulation of gas-particle flows in vertical risers, Ind. Eng. Chem. Res. 44 (16) (2005) 6022-6037.

R. Beetstra, M.A. Van Der Hoef, J.A.M. Kuipers, Drag force of intermediate Reynolds number flow past mono- and bidisperse arrays of spheres, AICHE J. 53 (2) (2007) 489501.

S. Benyahia, Verification and validation study of some polydisperse kinetic theories, J. Chem. Eng. Sci. 63 (23) (2008) 5672-5680.

S. Benyahia, Analysis of model parameters affecting the pressure profile in a circulating fluidized bed, AICHE J. 58 (2) (2012) 427-429.

G.A. Bokkers, J.A. Laverman, M. van Sint Annaland, J.A.M. Kuipers, Modelling of large-scale dense gas-solid bubbling fluidized beds using a novel discrete bubble model, J. Chem. Eng. Sci. 61 (17) (2006) 5590-5602.

Bona Lu,WeiWanga, Jinghai Lia, Searching for a mesh-independent sub-grid model for CFD simulation of gas-solid riser flows, J. Chem. Eng. Sci. 64 (15) (2009) 3437-3447.

S.H. Cho, H.G. Choi, J.Y. Yoo, Direct numerical simulation of fluid flow laden with many particles, Int. J. Multiphase Flow 31 (4) (2005) 435-451.

J. Ding, D. Gidaspow, A bubbling fluidisation model using theory of granular flow, AICHE J. 36 (4) (1990) 523-538.

N. Dombrowski, W.R. Johns, The aerodynamic instability and disintegration of viscous liquid sheets, J. Chem. Eng. Sci. 18 (3) (1963) 203-214.

S. Ergun, Fluid flow through packed columns, Chem. Eng. Prog. Symp. Ser. 48 (2) (1952) 89-94.

R. Garg, S. Tenneti, J. Mohd-Yusof, S. Subramaniam, Direct Numerical Simulation of Gas-Solids Flow Based on the Immersed Boundary Method, in: Thomas J. O'Brien, Sreekanth Pannala, Madhava Syamlal (Eds.),Computational Gas-Solids Flows and Reacting Systems: Theory, Methods and Practice 2011, pp. 245-276.

D. Gidaspow, Multiphase Flow and Fluidization: Continuum and Kinetic Theory Descriptions with Applications, Academic Press, Boston, 1994. 315-316.

R.J. Hill, D.L. Koch, A.J.C. Ladd, The first effects of fluid inertia on flows in ordered and random arrays of spheres, J. Fluid Mech. 448 (2001) 213-241.

R.J. Hill, D.L. Koch, A.J.C. Ladd, Moderate-Reynolds-number flows in ordered and random arrays of spheres, J. Fluid Mech. 448 (2001) 243-278. 
K. Hong, W.Wang, Q. Zhou, J.Wang, J. Li, An EMMS-based multi-fluid model (EFM) for heterogeneous gas-solid riser flows: part I. formulation of structure-dependent conservation equations, J. Chem. Eng. Sci. 75 (2012) 376-389.

H.H. Hu, N.A. Patankar, M.Y. Zhu, Direct numerical simulations of fluid-solid systems using the arbitrary Lagrangian-Eulerian technique, J. Comput. Phys. 169 (2) (2001) 427462.

Y. Igci, S. Pannala, S. Benyahia, S. Sundaresan, Validation studies on filtered model equations for gas-particle flows in risers, Ind. Eng. Chem. Res. 51 (4) (2012) 2094-2103.

J. Israelachvili, Intermolecular and Surface Forces, 3rd edition Academic Press, London, UK, 1991, ISBN 978-0-12-375182-9. (ALK. paper).

J.T. Jenkins, C. Zhang, Kinetic theory for identical, frictional, nearly elastic spheres, Phys. Fluids 14 (2002) 1228-1235.

S. Karimipour, T. Pugsley, Application of the particle in cell approach for the simulation of bubbling fluidized beds of Geldart A particles, J. Powder Technol. 220 (2012) 63-69.

J. Li, W. Ge, W. Wang, N. Yang, X. Liu, L. Wang, X. He, X. Wang, J. Wang, M. Kwauk, From Multiscale Modeling to Meso-Science, A Chemical Engineering Perspective Principles, Modeling, Simulation, and Application, Springer, Heidelberg New York Dordrecht London, 2013, http://dx.doi.org/10.1007/978-3-642-35189-1 (Library of Congress Control Number: 2012954048).

J. Li, M. Kwauk, Particle-Fluid Two Phase Flow, The Energy Minimization Multiscale Method, Metallurgy Industry Press, Beijing, 1994.

X. Liu, X. Xu, W. Zhang, Numerical simulation of dense particle gas two-phase flow using the minimal potential energy principle, J. Univ. Sci. Technol. Beijing Miner. Metall. Mater. 13 (4) (2006) 301-307.

B. Lu,W.Wang, J. Li, Searching for amesh-independent sub-gridmodel for CFD simulation of gas-solid riser flows, J. Chem. Eng. Sci. 64 (15) (2009) 3437-3447.

J. Ma,W. Ge, X.Wang, J.Wang, J. Li, High-resolution simulation of gas-solid suspension using macro-scale particle methods, J. Chem. Eng. Sci. 61 (21) (2006) 7096-7106.

T. McKeen, T. Pugsley, Simulation and experimental validation of a freely bubbling bed of FCC catalyst, J. Powder Technol. 129 (1-3) (2003) 139-152.

T. Mikami, H. Kamiya, M. Horio, Numerical simulation of cohesive powder behaviour in a fluidized bed, Chem. Eng. Sci. 53 (10) (1998) 1927-1940.

C.C. Milioli, F.E. Milioli, W. Holloway, K. Agrawal, S. Sundaresan, Filtered two-fluid models of fluidized gas-particle flows: new constitutive relations, AICHE J. 59 (9) (2013) 3265-3275. 
T. Nomura, T.J.R. Hughes, An arbitrary Lagrangian-Eulerian finite element method for interaction of fluid and a rigid body, J. Comput. Methods Appl. Mech. Eng. 95 (1) (1992) 115-138.

S. Pannala, M. Syamlal, T.J. O'Brien, Computational Gas-Solids Flows and Reacting Systems: Theory, Methods and Practice, Engineering Science Reference 2011. (13: 9781615206513).

[31] H.Y. Qi, F. Li, B. Xi, C. You, Modeling of drag with the Eulerian approach and EMMS theory for heterogeneous dense gas-solid two-phase flow, J. Chem. Eng. Sci. 62 (6) (2007) 1670-1681.

L. Schiller, A. Naumann, A Drag Coefficient Correlation, VDI Zeitung Publications, vol. 771935. 318-320.

J.P.K. Seville, C.D. Willett, P.C. Knight, Interparticle force in fluidization: a review, J. Powder Technol. 113 (3) (2000) 261-268.

N. Sharma, N. Patankar, A fast computation technique for the direct numerical simulation of rigid particulate flows, J. Comput. Phys. 205 (2) (2005) 439-457.

A. Samuelsberg, B.H. Hjertager, Computational modeling of gas/particle flow in a riser, AICHE J. 42 (6) (1996) 1536-1546.

M. Syamlal, W. Rogers, T.J. O'Brien, MFIX Documentation; Theory Guide, Technical Note, U.S. Department of Energy, Office of Fossil Energy, Morgantown Energy Technology Center, Morgantown, West Virginia, 1993.

M. Syamlal, W. Rogers, T.J. O'Brien, MFIX Documentation: Volume 1, Theory Guide, National Technical Information Service, Springfield, VA, 1993 (Appendix A).

M. Syamlal, T.J. O'Brien, Fluid dynamic simulation of O3 decomposition in 3 a bubbling fluidized bed, AICHE J. 49 (11) (2003) 2793-2801.

S. Tenneti, R. Garg, S. Subramaniam, Drag law for monodisperse gas-solid systems using particle-resolved direct numerical simulation of flow past fixed assemblies of spheres, Int. J. Multiphase Flow 37 (9) (2011) 1072-1092. 
CHAPTER 3: INVESTIGATION OF PARAMETERS CONTRIBUTING TO IMPROVEMENTS OF TWO FLUID MODELING OF A FLUDIZATION FLOW

\subsection{Abstract}

This study investigates the effect of combining a particle resolved direct numerical simulation (PR-DNS)-based method with several standard drag models available in the Two-Fluid Model (TFM) within the Multiphase Flow with Interphase Exchanges (MFIX) code. Implementation of hybrid method in simulation of the fluidization of fluid catalytic cracking (FCC) particles with air in a fluidized bed has shown improvements to the profile of solid volume fraction along the fluidization column. Different parameters such as Reynolds number, granular temperate, drag force between the solid and fluid phases, number of affected computational grid cells by the hybrid method and solid volume fraction are compared between the constituent and the hybrid models in our investigations. Qualitative comparisons were also performed for cluster formation prediction in the computational domain. A correlation for the drag force in different ranges of Reynolds number, solid volume fraction and the Ha parameter is driven.

\subsection{Introduction}

The TFM approach, developed by van Deemter and van der Laan (1961), is known as an economic way of simulating multiphase flows in large-scale fluidized bed risers (Pannala et al., 2011). This Eulerian-Eulerian approach treats solid and gas as continuous phases which can exchange mass, momentum, energy and other values depending on the nature and complexity of the problem at hand. This leads to significant reduction of memory and 
computational costs as compared to other widely exploited methods, such as the ParticleResolved Direct Numerical Simulation (Hu et al., 2001 and Nomura and Hughes, 1992) and Discrete Element Method (Tsuji et al. 1993). However, performance of the TFM in simulation of cohesive inter particle forces, such as electrostatic and van der Waals forces, must be carefully evaluated if the drag model used is lacking such provisions. Cohesive particles in Geldart $\mathrm{A}$ and $\mathrm{C}$ groups tend to agglomerate and create heterogeneous structures, called clusters. Li et al. (1991) defined clusters as the agglomeration of particles which can transform from strands in the center of the fluidized bed column to spheres in the vicinity of the solid boundaries of the fluidization column. Xu and Zhu (2011) defined clusters as dense clouds of particles having significantly more particles per unit volume than the surrounding dilute regions. According to researchers, such as Agrawal et al. (2001), Zhang and Vanderheyden (2002), McKeen and Pugsley (2003), Yang et al. (2003, 2004), Ye et al. (2005a,b, 2008), Qi et al. (2007), Wang J. et al. (2007 and 2009), Lu et al. (2009), Igci et al. (2012), and Li et al. (2013), clusters are responsible for significant reduction of the interfacial drag forces between the gas and solid phases. There have been several attempts to improve the performance of the MFIXTFM code by introducing more complex drag laws, which can consider the effect of subgrid-scale heterogeneous structures in TFM simulations, such as the filtered models of Igci et al. (2011) and Milioli et al. (2013). However, the constitutive models used in these filtered models were obtained from highly resolved simulations of kinetic theory-based TFM simulations in the absence of the cohesive interparticle forces. Abbasi et al. (2015) could fill this gap by inclusion of van der Waals cohesive interparticle forces into their proposed drag model and obtained significant improvement in solid concentration profile 
along the column of the fluidized bed. The proposed model by Abbasi et al., abbreviated as AGDSM, performs switch operations from a standard drag model to a Particle Resolved DNS-based drag model, named TGS and developed by Tenneti and Subramaniam (2011), wherever high van der Waals forces are present. Abbasi et al. (2015), expanded their investigations to three versions of drag models, where each version performs switch operations from a different standard model available in the MFIX simulation program to the TGS model.

Presently, MFIX is a widely known, reliable, and professionally established package for simulation of heat and mass transfer. MFIX accommodates a variety of drag models that can be used in TFM simulation of gas-solid particulate flows. Yet, the direct or indirect addition of models for particle-to-particle, attractive and repulsive forces to the transport equations solved in TFM, or to the available drag laws, is missing. According to Ye et al. (2005 a,b) and Seville et al. (2000), these forces could be formulated as $\overrightarrow{\mathrm{F}}_{\mathrm{ij}}{ }^{(\mathrm{c})}=\left(\mathrm{AR} / 6 \mathrm{~d}_{\mathrm{ij}}{ }^{2}\right)$ $\vec{n}_{\mathrm{ij}}$, where $\mathrm{F}_{\mathrm{ij}}{ }^{(\mathrm{c})}$ is the cohesive inter-particular force and $\mathrm{A}$ is the Hamaker constant $\left(\approx 10^{-19}\right.$ J) (Israelachvili, 1991), $\mathrm{R}$ is the radius of the mono-dispersed particles, $\mathrm{d}$ is the surface to surface distance between particles and $\vec{n}$ is the normal vector pointing from the center of particles $\mathrm{i}$ to the center of the particle $\mathrm{j}$. Further, they defined a scaling factor, $\varphi=\frac{\mathrm{AR}}{6 \mathrm{z}_{0}} \cdot \frac{1}{\mathrm{~K}_{\mathrm{B}} \Theta_{\mathrm{s}}}$, which is the ratio between the interparticle cohesive and destabilizing forces for $\mathrm{d} \leq 100 \mu \mathrm{m}$. In this definition, $\mathrm{K}_{\mathrm{B}}$ is the Boltzmann constant $\left(\mathrm{K}_{\mathrm{B}} \approx 1\right.$, Ye et al., 2005a), $\mathrm{Z}_{0}$ is the threshold for particles to be considered as clustered ( $\mathrm{Z}_{0} \approx 4 \mathrm{~nm}$, Seville et al., 2000) and $\mathrm{d}$ and $\Theta_{\mathrm{s}}$ are the diameter and granular temperature of the solid particles. 
Abbasi et al. (2015) showed that derivation of equations governing the particle motion could produce a cohesive index, $\mathrm{Ha}=\frac{\mathrm{A}}{\pi \rho \mathrm{d}_{\mathrm{p}}{ }^{2} \mathrm{~d}_{0} \Theta_{\mathrm{s}}}$, which can indicate the onset of cluster formation. In this definition, $\rho$ is the density of the solid particle, $d_{p}$ is the particle diameter, $\mathrm{d}_{0}$ is the surface to surface cut-off distance ( $\mathrm{d} 0=1 \times 10^{-4}$ times $\mathrm{dp}$ ), and $\Theta$ is the granular temperature. This definition, addressed the issue of direct cluster recognition based on the particle to particle distance, i.e., absence of particles as elements in the Eulerian-Eulerian framework. Abbasi et al. (2015), placed a conservative constraint in definition of cluster by disqualifying extremely dilute computational cells, i.e., $\varepsilon_{\mathrm{s}}<$ 0.001 , for cluster formation. In this definition $\varepsilon_{\mathrm{s}}$ is the volume fraction of the cell which is occupied by the solid phase. This constraint will be used frequently in the present paper to define the clustering identification and implementations.

In this study, we extended the investigation of Abbasi et al. (2015) to the study of factors which lead to significant improvements obtained by implementation of cohesioncontrolled switching operations as reported by Abbasi et al. (2015). The rest of the paper is organized as follows. First, governing equations related to TFM model and drag correlations are presented. Next, study of critical parameters before and after switch operations is presented and variation of drag forces against parameters such as cohesive index and other models is presented and followed by the examination of the proposed models in numerical simulations for flow in a fluidized bed. Finally, a conclusion is drawn on the effectiveness of the proposed model and the authors' perspective of the future work. 


\subsection{Governing Equations}

In the TFM, both the gas and the particulate phases are considered as interpenetrating continuous mediums. Complete derivations of the equations governing the two-fluid model can be found in the work of Gidaspow (1994). Here, the equations of the TFM model and drag coefficients used in the present paper are summarized in Table 3-1 and Table 3-2.

For equations listed in Table $3-1, \rho, \overrightarrow{\mathrm{u}}, \varepsilon, \mathrm{g}, \overline{\bar{\tau}}_{\mathrm{k}}, \beta, \mathrm{p}, \lambda, \mu, \Theta_{\mathrm{s}}$, and $\mathrm{e}_{\mathrm{ss}}$ represent density, velocity vector, volume fraction, acceleration of gravity, shear stress tensor, momentum exchange coefficient, thermodynamic pressure, second coefficient of viscosity (or bulk viscosity), dynamic viscosity, granular temperature of the solid phase and particleparticle restitution coefficient . Here, the $\mathrm{e}_{\mathrm{ss}}$ is set to 0.9 according to Jenkins and Zhang (2002) and Benyahia (2012). In addition, k and l serve as identifiers for gas and solid phases. However, in Eq. (2), identifiers are phase specific, where if k refers to one of the phases (e.g., fluid), then 1 can only refer to the solid, and vice versa. In this work, the second coefficient of viscosity for the gas phase is set to zero, as suggested by Lu et al. (2009). For full description of the variables and the approach to find the cohesive index, readers are refer to the chapter 2 of the present dissertation. 
Table 3-1 Governing Equation for the TFM

Continuity balance equations

$$
\frac{\partial\left(\rho_{\mathrm{k}} \varepsilon_{\mathrm{k}}\right)}{\partial \mathrm{t}}+\nabla \cdot\left(\rho_{\mathrm{k}} \varepsilon_{\mathrm{k}} \overrightarrow{\mathrm{u}}_{\mathrm{k}}\right)=0,
$$

Momentum balance equation

$$
\frac{\partial\left(\rho_{\mathrm{k}} \varepsilon_{\mathrm{k}} \overrightarrow{\mathrm{u}}_{\mathrm{k}}\right)}{\partial \mathrm{t}}+\nabla \cdot\left(\rho_{\mathrm{k}} \varepsilon_{\mathrm{k}} \overrightarrow{\mathrm{u}}_{\mathrm{k}} \overrightarrow{\mathrm{u}}_{\mathrm{k}}\right)=-\varepsilon_{\mathrm{k}} \nabla \mathrm{p}_{\mathrm{k}}+\varepsilon_{\mathrm{k}} \rho_{\mathrm{k}} \overrightarrow{\mathrm{g}}+\nabla \cdot\left(\varepsilon_{\mathrm{k}} \overline{\overline{\mathrm{\tau}}}_{\mathrm{k}}\right)+\beta\left(\overrightarrow{\mathrm{u}}_{\mathrm{l}}-\overrightarrow{\mathrm{u}}_{\mathrm{k}}\right),
$$

Stress tensor

$$
\overline{\bar{\tau}}_{\mathrm{k}}=\left[\lambda_{\mathrm{k}} \nabla \cdot \overrightarrow{\mathrm{u}}_{\mathrm{k}}\right] \overline{\overline{\mathrm{I}}}+2 \mu_{\mathrm{k}}\left[\frac{1}{2}\left[\nabla \overrightarrow{\mathrm{u}}_{\mathrm{k}}+\left(\nabla \overrightarrow{\mathrm{u}}_{\mathrm{k}}\right)^{\mathrm{T}}\right]-\frac{1}{3} \nabla \cdot \overrightarrow{\mathrm{u}}_{\mathrm{k}} \overline{\overline{\mathrm{I}}}\right] .
$$

Total pressure term in solid phase

$$
\mathrm{p}_{\mathrm{s}}=\mathrm{p}_{\mathrm{g}}+\mathrm{P}_{\mathrm{s}} .
$$

Solid phase pressure

$$
P_{\mathrm{s}}=\Theta_{\mathrm{s}}\left[1+2\left(1+\mathrm{e}_{\mathrm{ss}}\right) \varepsilon_{\mathrm{s}} \mathrm{g}_{0_{\mathrm{ss}}}\right] .
$$

Bulk viscosity in solid phase

$$
\lambda_{\mathrm{s}}=\rho_{\mathrm{s}} \mathrm{d}_{\mathrm{p}}\left(\mathrm{e}_{\mathrm{ss}}+1\right) \frac{4 \varepsilon_{\mathrm{s}}^{2} \sqrt{\Theta_{\mathrm{s}}} \mathrm{g}_{0}}{3 \sqrt{\pi}},
$$

Dynamic viscosity in solid phase

$$
\mu_{\mathrm{s}}=\frac{5 \sqrt{\left(\pi \Theta_{\mathrm{s}}\right)} \rho_{\mathrm{s}} \mathrm{d}_{\mathrm{p}}}{48\left(\mathrm{e}_{\mathrm{ss}}+1\right) \mathrm{g}_{0}}\left[\left(1+\frac{4}{5}\left(\mathrm{e}_{\mathrm{ss}}+1\right) \varepsilon_{\mathrm{s}} \mathrm{g}_{0}\right]^{2}+\left(\frac{4 \varepsilon_{\mathrm{s}}^{2} \rho_{\mathrm{s}} \mathrm{d}_{\mathrm{p}} \mathrm{g}_{0}\left(1+\mathrm{e}_{\mathrm{ss}}\right) \sqrt{\Theta_{\mathrm{s}}}}{5 \sqrt{\pi}}\right),\right.
$$

Diffusion coefficient of the granular temperature in solid phase

$$
\mathrm{k}_{\boldsymbol{\theta}_{\mathrm{s}}}=\frac{150 \rho_{\mathrm{s}} \mathrm{d}_{\mathrm{s}} \sqrt{\left(\Theta_{\mathrm{s}} \pi\right)}}{384\left(1+\mathrm{e}_{\mathrm{ss}}\right) \mathrm{g}_{0}}\left[1+\frac{6}{5} \varepsilon_{\mathrm{s}} \mathrm{g}_{0}\left(1+\mathrm{e}_{\mathrm{ss}}\right)\right]^{2}+2 \rho_{\mathrm{s}} \boldsymbol{\varepsilon}_{\mathrm{s}}^{2} \mathrm{~d}_{\mathrm{s}}\left(1+\mathrm{e}_{\mathrm{ss}}\right) \mathrm{g}_{0} \sqrt{\frac{\Theta_{\mathrm{s}}}{\pi}},
$$

Collisional energy dissipation in solid phase

$$
\gamma_{\theta_{s}}=3\left(1-e_{s s}^{2}\right) \rho_{s} \varepsilon_{s}^{2} \Theta_{s} g_{0}\left(\frac{4 \sqrt{\Theta_{s}}}{d_{s} \sqrt{\pi}}-\nabla \cdot \vec{u}_{s}\right)
$$


Table 3-2 Governing equations for the existing drag models used for switching procedure O’Brien-Syamlal Drag Model (Syamlal et al., 1993)

$$
\begin{aligned}
& \beta=\frac{3 \varepsilon_{g} \varepsilon_{S} \rho_{g}}{4 V_{r s}^{2} d_{p}} C_{d 0}\left(\frac{R e_{p}}{V_{r s}}\right)\left|V_{g}-V_{s}\right| \\
& V_{r s}=0.5\left(A-0.06 \operatorname{Re}_{p}+\sqrt{\left(0.06 R_{p}\right)^{2}+0.12 \operatorname{Re}_{p}(2 B-A)+A^{2}}\right. \\
& A=\varepsilon_{g}^{4.14} \\
& B=\left\{\begin{array}{l}
0.8 \varepsilon_{g}^{1.28} \text { for } \quad \varepsilon_{g} \leq 0.85 \\
\varepsilon_{g}^{2.65} \text { for } \quad \varepsilon_{g}>0.85 \\
C_{d 0}\left(\frac{R_{p}}{V_{r s}}\right)=\left(0.63 \sqrt{\frac{R_{p}}{V_{r s}}}+4.8\right)^{2} \times \frac{V_{r s}}{R_{p}}
\end{array}\right.
\end{aligned}
$$

Gidaspow Drag Model (Gidaspow (1994))

$$
\begin{aligned}
& \beta=\left\{\begin{array}{lll}
150 \frac{\varepsilon_{\mathrm{s}}^{2} \mu_{\mathrm{g}}}{\mathrm{d}_{\mathrm{p}}{ }^{2} \varepsilon_{\mathrm{g}}}+1.75 \varepsilon_{\mathrm{s}} \frac{\rho_{\mathrm{g}}}{\mathrm{d}_{\mathrm{p}}}\left|\mathrm{V}_{\mathrm{g}}-\mathrm{V}_{\mathrm{s}}\right| & \text { for } & \varepsilon_{\mathrm{g}} \leq 0.8 \\
0.75 \mathrm{C}_{\mathrm{d} 0} \frac{\varepsilon_{\mathrm{s}} \varepsilon_{\mathrm{g}} \rho_{\mathrm{g}}}{\mathrm{d}_{\mathrm{p}}}\left|\mathrm{V}_{\mathrm{g}}-\mathrm{V}_{\mathrm{s}}\right| \varepsilon_{\mathrm{g}}{ }^{-2.65} & \text { for } & \varepsilon_{\mathrm{g}}>0.8
\end{array}\right. \\
& \mathrm{C}_{\mathrm{d} 0}=\left\{\begin{array}{lll}
0.44 & \text { for } & \mathrm{Re} \geq 1000 \\
\frac{24}{\operatorname{Re}_{\mathrm{p}}}\left(1+0.15 \mathrm{Re}_{\mathrm{p}}^{0.687}\right) & \text { for } & \mathrm{Re}<1000
\end{array}\right.
\end{aligned}
$$

Wen-Yu Drag Model (Wen and Yu,1966 and Xu et al., 2012)

$$
\begin{aligned}
& \beta=0.75 \mathrm{C}_{\mathrm{d} 0} \frac{\varepsilon_{\mathrm{s}} \varepsilon_{\mathrm{g}} \rho_{\mathrm{g}}}{\mathrm{d}_{\mathrm{p}}}\left|\mathrm{V}_{\mathrm{g}}-\mathrm{V}_{\mathrm{s}}\right| \varepsilon_{\mathrm{g}}{ }^{-2.65} \\
& \mathrm{C}_{\mathrm{d} 0}=\left\{\begin{array}{lll}
0.44 & \text { for } & \mathrm{Re} \geq 1000 \\
\frac{24}{\operatorname{Re}_{\mathrm{p}}}\left(1+0.15 \operatorname{Re}_{\mathrm{p}}{ }^{0.687}\right) & \text { for } & \operatorname{Re}<1000
\end{array}\right.
\end{aligned}
$$


Table 3-2 continue

TGS Drag Model (Tenneti and Subramaniam, 2011)

$$
\begin{aligned}
& F\left(\varepsilon_{\mathrm{s}}, \operatorname{Re}_{\mathrm{p}}\right)=\frac{\mathrm{F}_{\text {isol }}\left(\mathrm{Re}_{\mathrm{p}}\right)}{\left(1-\varepsilon_{\mathrm{s}}\right)^{3}}+\mathrm{F}_{\varepsilon_{\mathrm{s}}}\left(\varepsilon_{\mathrm{s}}\right)+\mathrm{F}_{\varepsilon_{\mathrm{s}}, \mathrm{Re}_{\mathrm{p}}}\left(\varepsilon_{\mathrm{s}}, \operatorname{Re}_{\mathrm{p}}\right) \\
& \mathrm{F}_{\text {isol }}\left(\operatorname{Re}_{\mathrm{p}}\right)=1+0.15 \operatorname{Re}_{\mathrm{p}}^{0.687}, \\
& \mathrm{~F}_{\varepsilon_{\mathrm{s}}}\left(\varepsilon_{\mathrm{s}}\right)=\frac{5.81 \varepsilon_{\mathrm{s}}}{\left(1-\varepsilon_{\mathrm{s}}\right)^{3}}+0.48 \frac{\varepsilon_{\mathrm{s}}{ }^{1 / 3}}{\left(1-\varepsilon_{\mathrm{s}}\right)^{4}}, \\
& \mathrm{~F}_{\varepsilon_{\mathrm{s}}, \operatorname{Re}_{\mathrm{p}}}\left(\varepsilon_{\mathrm{s}}, \operatorname{Re}_{\mathrm{p}}\right)=\varepsilon_{\mathrm{s}}{ }^{3} \operatorname{Re}_{\mathrm{p}}\left(0.95+\frac{0.61 \varepsilon_{\mathrm{s}}{ }^{1 / 3}}{\left(1-\varepsilon_{\mathrm{s}}\right)^{2}}\right) \\
& \beta=18 \mu_{\mathrm{g}} \varepsilon_{\mathrm{s}} \varepsilon_{\mathrm{s}} \frac{\mathrm{F}\left(\varepsilon_{\mathrm{s}}, \operatorname{Re}_{\mathrm{p}}\right)}{\mathrm{d}_{\mathrm{p}}{ }^{2}}
\end{aligned}
$$

\subsection{Simulation Methodology}

Chapter 2 of this dissertation was devoted to a study about performance of three versions of hybrid models, AGDSM1, AGDSM2, and AGDSM3 in TFM simulations of a fluidization flow. It was shown that hybrid models outperformed their constituent models in terms of agreement with the experimental data. Nominated results of the simulations are displayed in Figure 3-1 for the purpose of better referencing. Additional results including quantitative levels of improvements in terms of relative error (imp.Err.rel.), maximum error (imp.Err.max), coefficient of determination (imp. $\mathrm{R}^{2}$ ), are available in the Chapter 2 of this dissertation. 


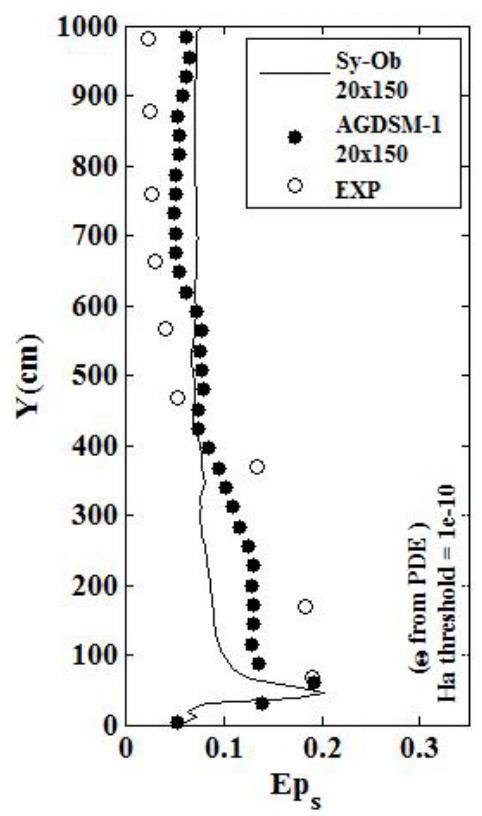

(a)

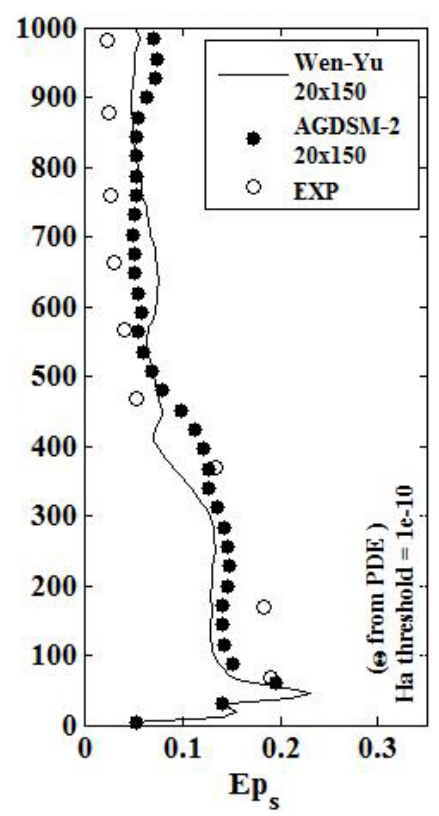

(b)

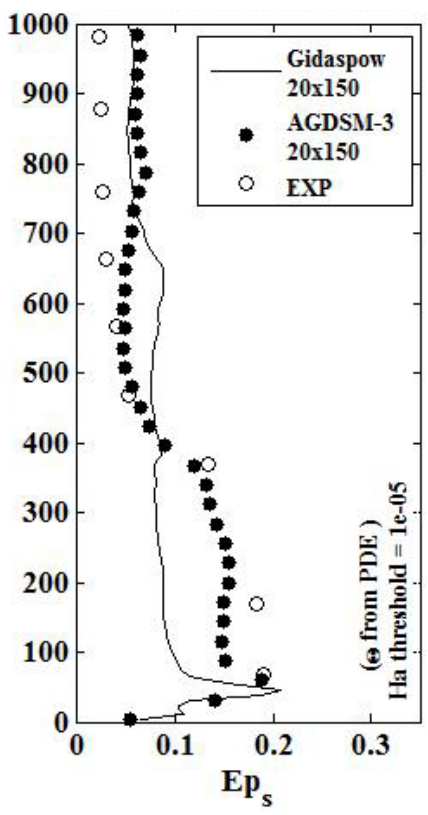

(c)

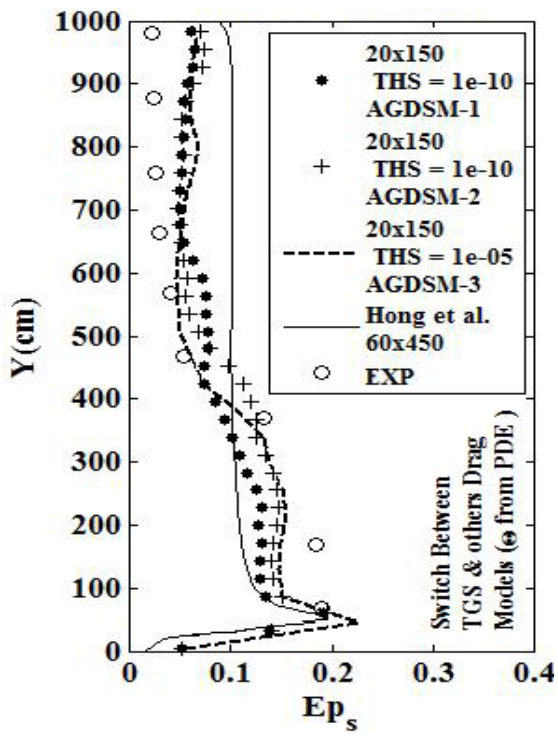

(d)

Figure 3-1 Optimal MFIX-TFM simulations by versions of the AGDSM model, (a) to (c) are versions of the AGDSM model versus corresponding standard models, (d): best result from AGDSM1-3 versions and the profile of Hong et al. (2012) 
In the present study, the simulation results of the Abbasi et al. (2015) were used to investigate about parameters which contributed to the performance improvements. We performed post processing of the results to ensure that mean quantities reached steady values within averaging time intervals. Extraction of data from post-processing module of MFIX along with, time and area averaging, thresholding to find switch operations and zones of clustering, and visualizations were performed using Matlab scripts.

A visualization approach is used here to qualitatively visualize the difference between distributions of filed variables such as solid concentration, drag force, and switch operations inside the computational domain before and after the standard models were combined with the TGS model. In this approach the time-averaged quantities $\left(<\Phi>=\frac{1}{\mathrm{M}}\left(\sum_{\mathrm{t}=\mathrm{L}}^{\mathrm{L}+\mathrm{M}} \Phi(\mathrm{x}, \mathrm{t})\right)\right.$ of any field variable represented by $\Phi$ over $\mathrm{M}$ number of time steps was used to represent mean quantity inside the computational domain. Here L represents the time step for start of averaging and was wisely selected to represent the start of statistically steady regime of the flow, where mean of quantities have reached an almost steady state with negligible variation. In this work the last 1000 time steps represent the steady state condition of the flow inside the computational domain.

Part of the analysis in this paper is aimed to compare the values of the normalized drag forces between the constituent and hybrid drag model. Using the data obtained by the constituent and hybrid model, it was possible to discover how the normalized drag force would vary with and without use of switch operations controlled by the cohesive index. This analysis helped to discover how the issue of over-prediction of drag forces by the 
standard drag models could be addressed by controlled switch operations inside the hybrid models using the cohesive index, Ha. Equation (3.25) displays the definition of the normalized drag force used in our analysis.

$$
\mathrm{F}_{\text {drag.norm }}=\frac{\mathrm{F}_{\text {model }}}{\mathrm{F}_{\text {Stokes }}}=\frac{\mathrm{F}_{\text {model }}}{3 \pi \mu_{\mathrm{g}} \varepsilon_{\mathrm{g}} \mathrm{d}_{\mathrm{p}}\left|\mathrm{V}_{\mathrm{g}}-\mathrm{V}_{\mathrm{s}}\right| \Delta \mathrm{V}}=\frac{\beta \mathrm{d}_{\mathrm{p}}}{32 \mu_{\mathrm{g}} \varepsilon_{\mathrm{g}}}
$$

In this definition $\mu_{\mathrm{g}}, \varepsilon_{\mathrm{g}}, \mathrm{d}_{\mathrm{p}}, \Delta \mathrm{V}, \beta$, and $\left|\mathrm{V}_{\mathrm{g}}-\mathrm{V}_{\mathrm{s}}\right|$ represent the viscosity and volume fraction of the gas, particle diameter, drag coefficient, and the relative velocity between the gas and solid phases, respectively. Here, the $\mathrm{F}_{\text {Stokes }}$ is the drag on a single sphere which has the same volume as of the solid volume fraction of the computational cell.

\subsection{Results and Discussion}

In the first attempt to evaluate the cluster prediction inside the computational domain, we qualitatively visualized the time-averaged distributions of solid concentration in the domain for the standard models before and after being combined with the TGS model. The contours of $\varepsilon_{\mathrm{s}}$ in Figure 3-2(a-d) show significantly different concentrations of the solid phase between the constituent models. In special, Figure 3-2(a-c) shows that constituent models presented higher concentrations in the upper half section of the fluidization column. As expected from the similarity of profiles in Figure 3-1(d), the three hybrid models in Figure 3-2(e-g) presented very similar concentrations in the domain. These counters show higher solid concentrations close to the inlet and on the solid boundaries which are in significantly better agreements with the experimental data, as shown by empty circles in Figure 2-6(d). Additional confirmation was obtained by comparing these contours with the contours displayed by Benyahia (2012) and Hong et 
al. (2012), who used improved drag models. In addition, this similarity exists in Figure 3-2(d) for the Wen-Yu model, and is consistent with similarity of profiles in Figure 3-1(b).

In another observation, regions of higher solid concentration, which may represent cluster, are observed on the axis of the computational domain for the Syam-O'brien and TGS models. Proof of the existence of such structures can only be provided by comparison with images of the flow in a fluidization experiments or the data obtained by optical probes. One explanation for such observations, which led to overestimation of solid concentration in the upper half region of the domain, can be overestimation of airsolid interactions for the high Reynolds number regions of the domain, i.e., the regions surrounding the axis of fluidized bed, described as the core region of the flow with high values of voidage by Li and Kuwak (1994) and Xu and Zhu (2011). 


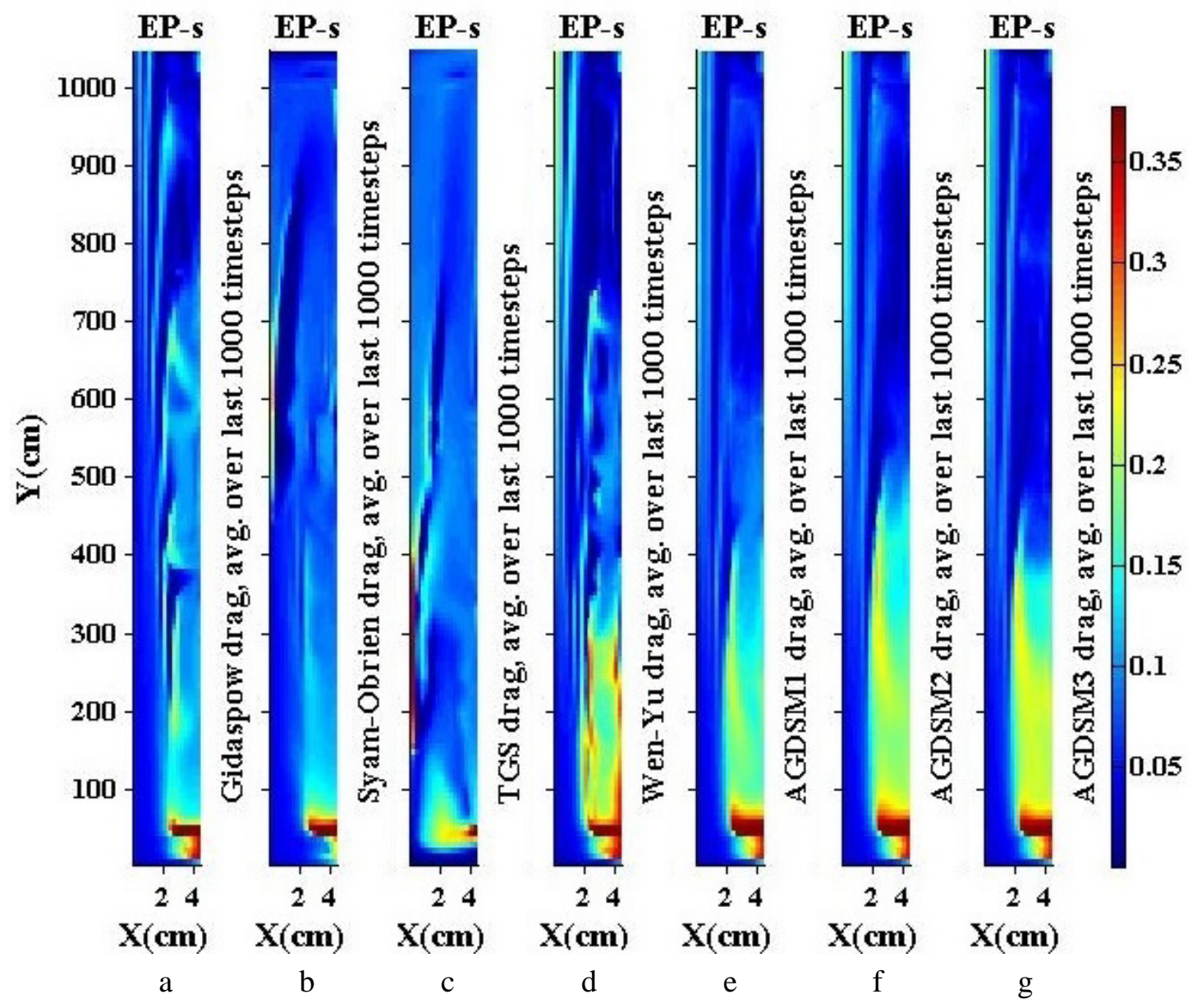

Figure 3-2 Contour of solid volume fraction averaged over the last 1000 time steps

Figure 3-3 shows a quantitative comparison between the frequencies of switch operations performed by the hybrid models, recalled as modifications, and potential of modifications for the constituent models, against time. Strikingly, constituent models required significantly larger modifications, as compared to the hybrid models. These results demonstrate that modifications that were required to improve the agreement between the numerical results and the experimental data have taken place in the hybrid models and only small number of cells would experience switching operations operated by the algorithm of the drag model. 


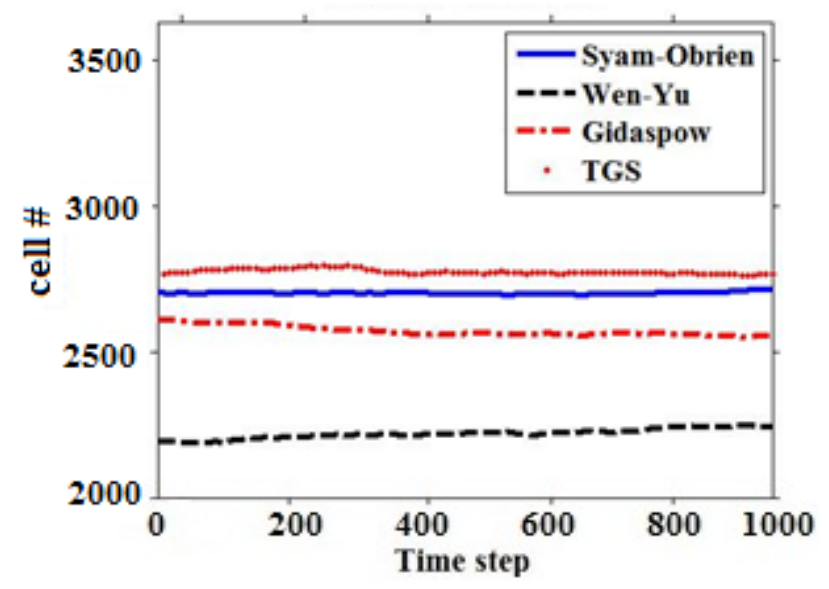

(a)

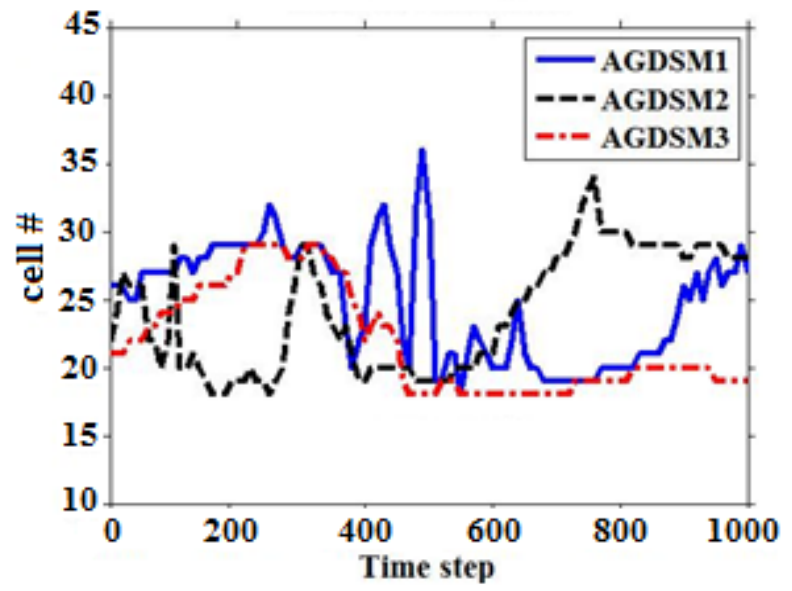

(b)

Figure 3-3 Frequency of switch operations. (a) constituent drag models, (b) proposed AGDSM model versions in optimal cases

To better visualize the switching operations associated with cluster predictions, Figure 3-4 shows the number of computational cells which met the criteria of cluster formation, Ha > Ha-THS, in our TFM simulations. This figure shows the possibility of further modification to both the constituent and hybrid models used in this study. This can also be interpreted as the degree of effectiveness of the switching operations between the TGS and the standard models. In these results, the maximum of possibility is shown by the red color and is defined as occurrence of switch operation. Therefore, the number of switch operations can be readily observed from these contours. According to Figure 3-4, computational analysis the Wen-Yu model was subject to the smallest amount of modifications among the constituent models. In the case of hybrid models, differences between variable contours are indistinguishable, despite the fact that different thresholds were used for the models, i.e., Ha $>1 \times 10^{-10}$ for the AGDSM1 and AGDSM2 and Ha > $1 \times 10^{-5}$ for the AGDSM3. Required modifications for hybrid models are limited to a zone 
of the domain in vicinity of the inlet port. According to Figure 3-2, this zone of the domain contained the maximum solid concentration in our simulations.

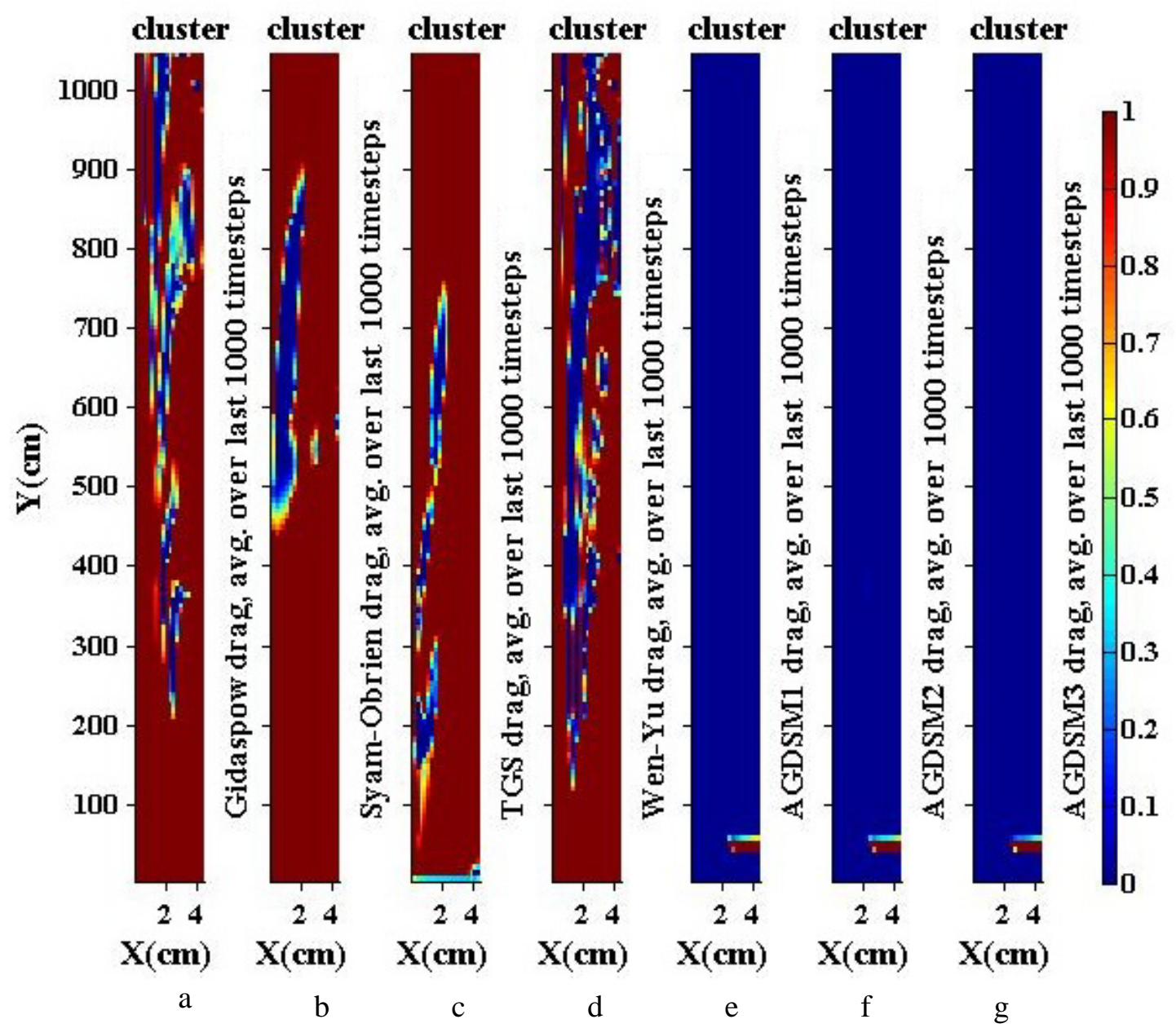

Figure 3-4 Modification due to cluster formation (cells meeting the criteria of Ha $>$ Ha-THS for switch operations)

Figure 3-5 shows the time-averaged magnitude of the drag force inside the computational domain for all the models investigated in this study. All models show significantly large drag magnitudes in the lower sections of the computational domain. The three hybrid models produced very similar magnitudes of drag force in the entire domain. A small 
area with higher drag force magnitude is observed in the AGDSM1 model close to the inflation (height $\approx 470 \mathrm{~cm}$ ) point in contour e, which is different in contours $\mathrm{f}$ and g. The smaller magnitudes of drag force in the upper half region of the domain in Figure 3-5(eg) are due to less concentration of solids and this can be observed in Figure 3-5(a and d). Surprisingly, among the constituent models, the Wen-Yu model, Figure 3-5(d), has produced the most similar contour to the contours of the hybrid model Figure 3-5(e-g). Furthermore, in the case of Syam-O'Brien and TGS models, Figure 3-5(b) and Figure 3-5(c) exhibit larger values of the drag in the upper half section of the computational domain. This overestimation of the drag force is worsened by presence of large values of drag force stretched along the axis of the computational domain in Figure 3-5(b), 


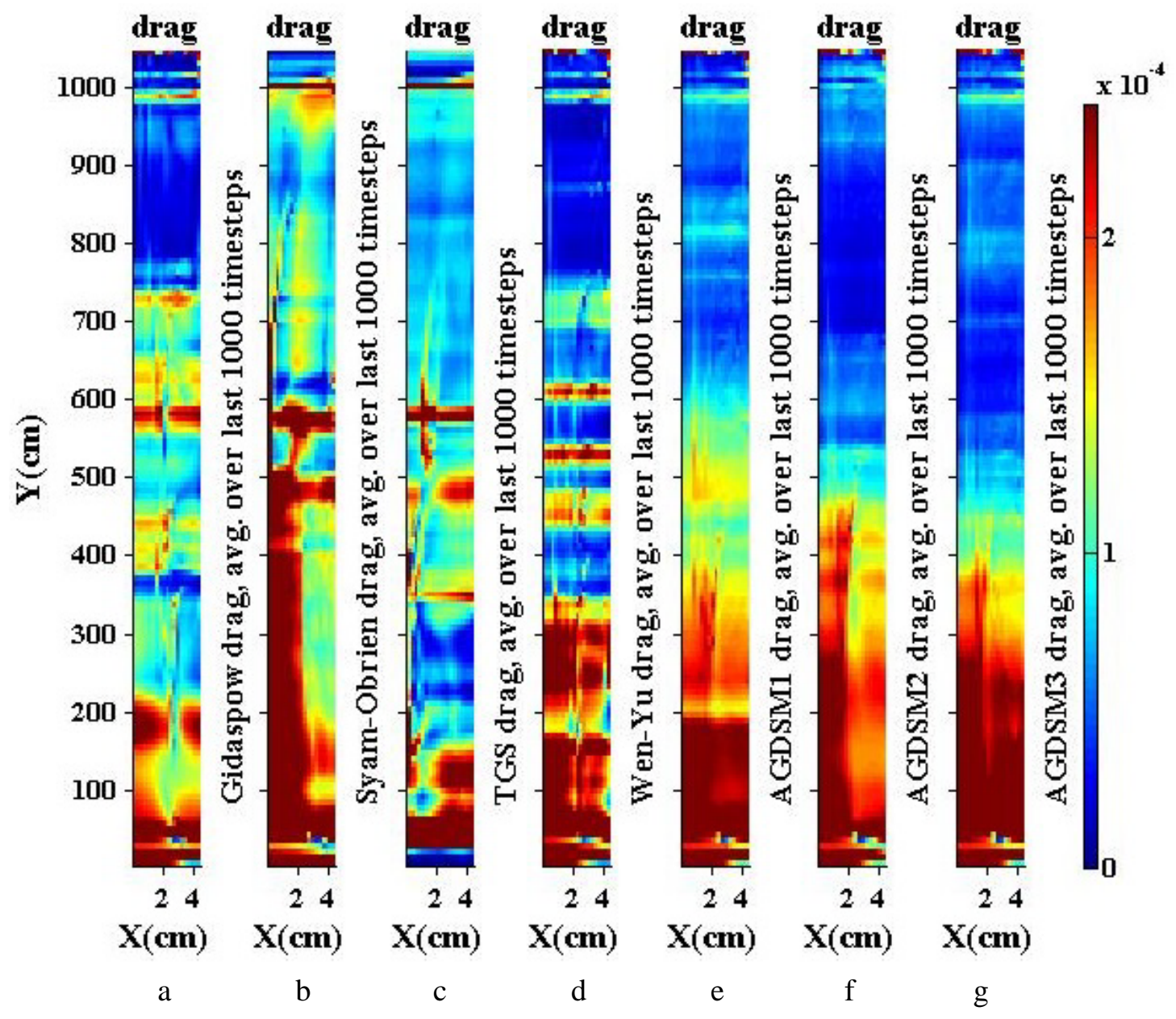

Figure 3-5 Contour of drag force averaged over the last 1000 time steps

Further investigations were performed to compare the constituent and hybrid models in regards to parameters such as, drag force, normalized drag force $\left(\mathrm{F}_{\text {drag }} / \mathrm{F}_{\mathrm{St}}\right)$, granular temperature, and cohesive index. These parameter were time-averaged over the last 1000 time steps of the simulation and the purpose of these comparisons was to investigate about the underlying factors that control the modifications to the constituent models.

Figure 3-6 shows the comparison between models in regards to quantities such as the drag force and normalized drag force that are spatially averaged over the last 1000 time 
steps of the simulations. The left and right columns show the results for the constituent and hybrid models, respectively. Figure $3-6(a \& c)$ shows that among the constituent models, the Syam-O'Brien model produced the largest values of the absolute and normalized drag forces in the simulations. The TGS model produced the smallest amount of the drag force in most of the time steps, while this model produced the second highest values of the normalized drag force. The Gidaspow and Wen-Yu models produced very similar values of the normalized drag force, which are the smallest values among all the constituent and hybrid models, as is obvious from the Figure 3-6(d). Figure 3-6(c) shows that among the hybrid models under optimal Ha-THS, the AGDSM1 drag model (combination of Syam-O’Brien and TGS) produced slightly higher values of the drag in its absolute and normalized form. However, small differences exist between profiles in Figure 3-6(b\&d) and $F_{\text {drag.norm }}$ is less than one for all the hybrid models over the entire range of time steps. The physical interpretation of this fact is that smaller drag force was obtained in each computational cell in the TFM approach, compared to the situation where a single sphere with the same volume fraction $\left(\varepsilon_{s}\right)$ of the computational cell would be placed inside the computational cell.

Comparison between the profiles of normalized drag force in Figure 3-6(a) can help to understand the factors that resulted in significantly higher improvements of accuracy for models such as Syam-O’Brien and Gidaspow. The improvement of AAPD criteria (defined as average absolute percentage deviation) was reported as 32 percent and 26 percent for the Syam-O'Brien and Gidaspow models, respectively. In this light, switching to the TGS model created the largest reduction of the drag force magnitude for the Syam- 
O’Brien drag model and the second largest reduction happened for the Wen-Yu model. However, it must be considered that changes in the models depended on both the frequency and impact of switching operations. Figure 3-3 showed previously that the maximum of switching frequencies happened for the Syam-O'Brien model and this quantity was significantly higher for the Gidaspow model in comparison to the Wen-Yu model. A better explanation of these facts can be obtained by comparing each hybrid model against its constituent models. In fact, by considering the fact that more accurate drag forces were obtained by the hybrid models, the amount of modification for each constituent model can be quantified. Figure 3-7(a) shows the difference between hybrid and constituent models in terms of the magnitude of drag force. In this figure, the greatest difference is observed for the Syam-O'Brien model. This difference is small for the TGS model, and cannot explain the 52 percent of modification that was obtained for AAPD criteria for the TGS model. Hereby, it must be noted that equality of spatially averaged drag coefficient must not be interpreted as equality of the drag magnitude for the entire domain. Examples can be significant differences in contour of drag force magnitudes (Figure 3-5), and other quantities, such as solid volume fraction (Figure 3-2) and the contour of modification (Figure 3-4).

Normalized drag force was selected for comparison between hybrid and constituent models in Figure 3-7(b). This quantity is a more appropriate criteria since it includes the solid volume fraction in its formulation, i.e., Eq.(3.25). Comparison of normalized drag force values in Figure 3-7(b) reveals the big differences between the Syam-O'Brien and AGDSM1 models, and TGS and AGDSM3 models. In addition, this figure shows smaller 
differences between the Gidaspow and AGDSM3 models and the smallest difference is shown for the Wen-Yu model. Furthermore, the relative differences are in better agreement with the percentages of modifications obtained for the AAPD criterion.

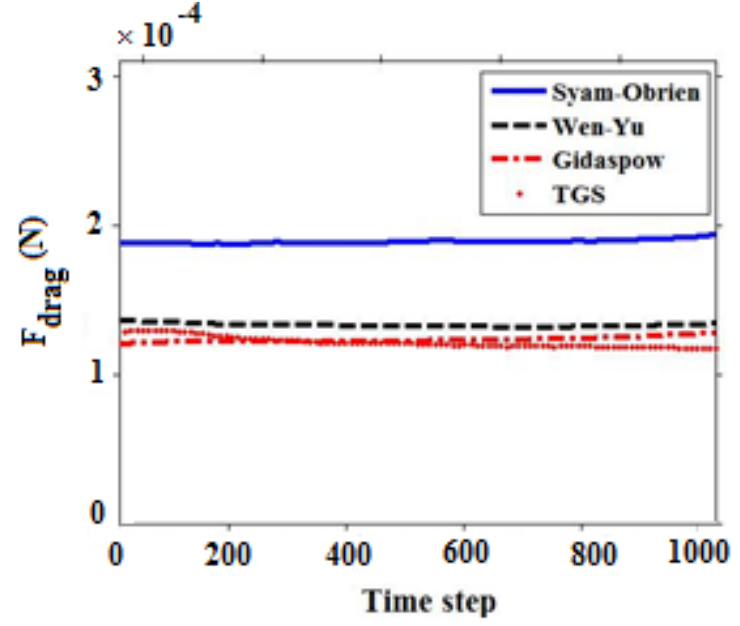

(a)

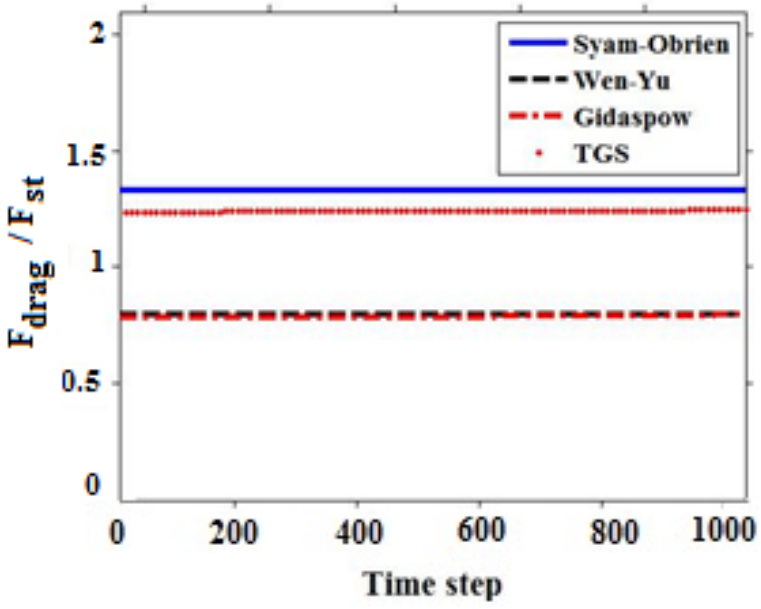

(c)

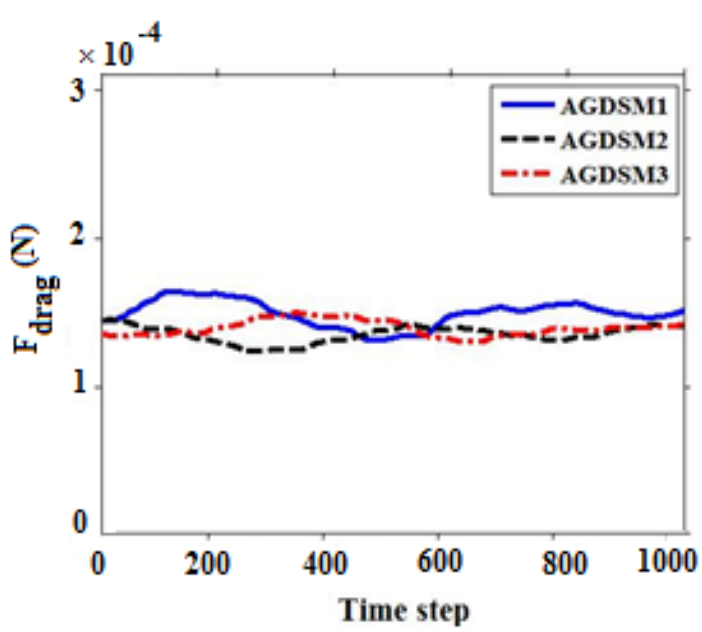

(b)

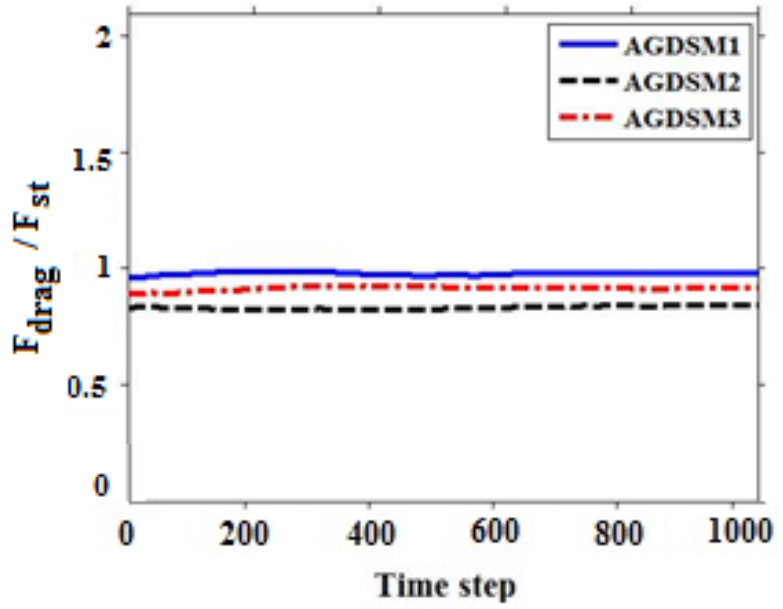

(d)

Figure 3-6 Averaged values of drag forces (a-b) and normalized drag forces (c-d) 


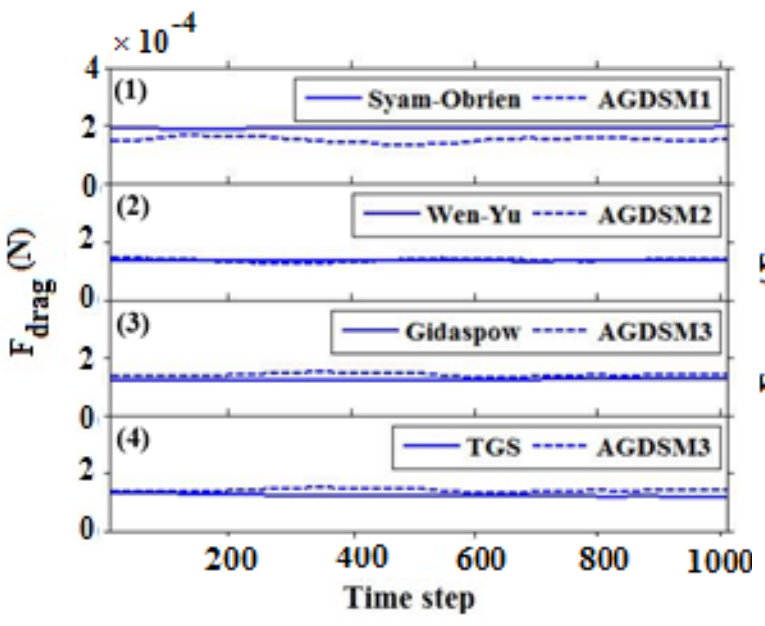

(a)

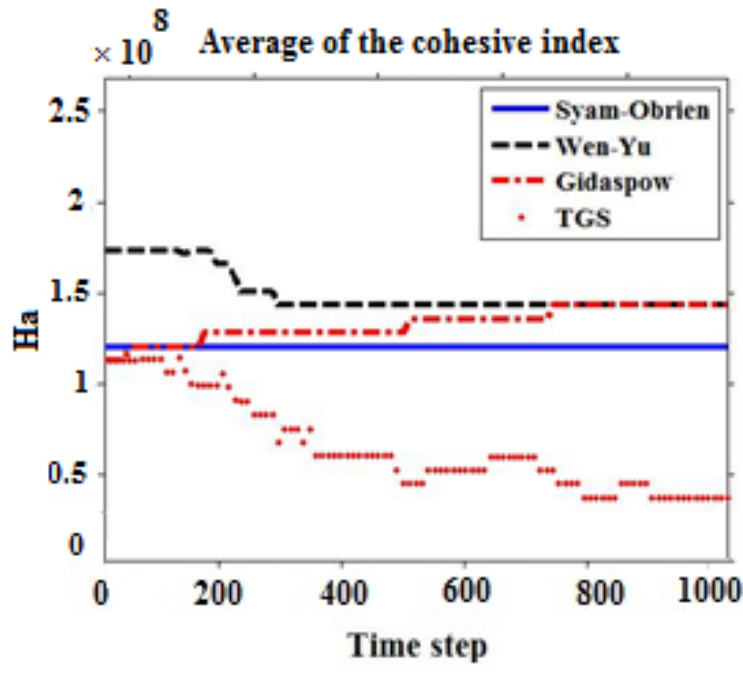

(C)

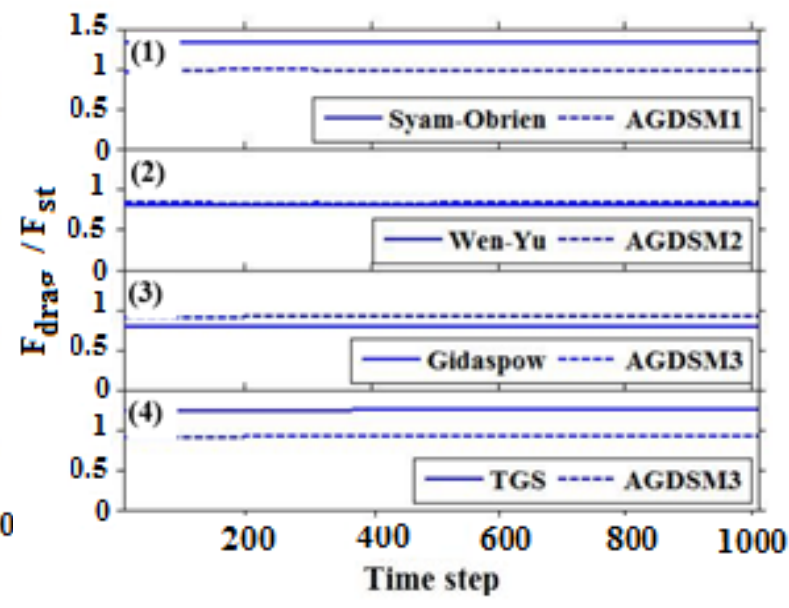

(b)

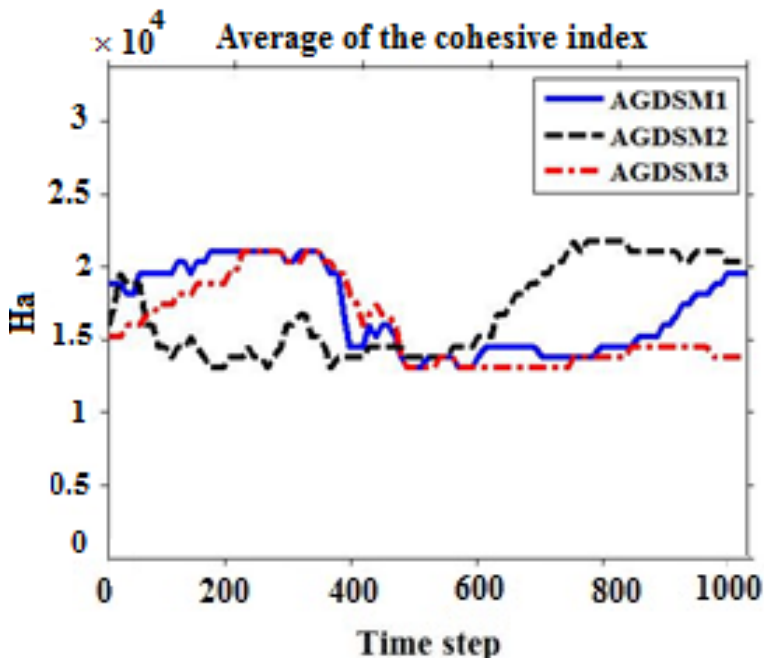

(d)

Figure 3-7 Comparisons of spatially-averaged variables over the 100 last time steps: (a) drag force (b) normalized drag force.

Another quantity displayed in Figure 3-7(c\&d) is the average of cohesive index which is in inverse relation with the number of switching operations performed by the models. Figure 3-7(c) shows that average of the potential switching operations than can be performed during simulation of constituent models. Here, the smallest values were obtained by the TGS model. Therefore, switching to the TGS model resulted in smaller 
values of Ha and reduced the probability of occurrence of more switching operations. This self-stabilizing procedure could eventually result in significantly smaller values of Ha and number of cells that met the criteria of switching operations in the AGDSM models, as is shown in Figure 3-7(d) and Figure 3-3, respectively. Consequently, larger average values of granular temperature were obtained in the domain and cluster predictions were eliminated from most regions of the domain, except the vicinity of the inlet port. As a consequence, profiles in Figure 3-7(d) show that spatially-averaged values of Ha are similar for all three hybrid models. Based on these results, huge differences exist between Figure 3-7(c) and Figure 3-7(d), and relative differences between constituent and hybrid models cannot be related to the degree of modifications obtained for the constituent models.

\subsection{Conclusion}

In this chapter, simulation results presented in chapter 2 were analyzed and factors contributing to improvements of numerical results were investigated. We presented qualitative and quantitative investigations about drag force, normalized drag force, and frequency of switching operations performed by three hybrid models. It was shown that switching to the TGS model with the lowest values of drag force, and the mechanism that controlled switch operations were the main contributing factors for performance improvements (AAPD reductions) of hybrid models. The switching operations were controlled in a self-stabilizing procedure and resulted in dramatic reduction of cohesive index and number of cells that met the criteria of cluster formation. This has led to the elimination of most of the cluster formations from the domain by all hybrid models and 
cluster predictions were limited to extremely dense regions of the domain in vicinity of the solid inlet port. Additionally, we found that magnitude of normal drag force was less than one for all three hybrid models and adjustment of constituent models to hybrid models was proven to be proportional to performance improvements.

\subsection{References}

Abbasi Baharanchi A., Gokaltun S., Dulikravich G.S., 2015, Performance Improvement of Existing Drag Models in Two-Fluid Modeling of Gas-Solid Flows Using a PR-DNS Based Drag Model, Journal of Powder Technology, Volume 220, pp 63-69

Agrawal K. , Loezos P.N., Syamlal M., Sundaresan S., 2001, The role of meso-scale structures in rapid gas solid flows, Journal of Fluid Mech. (2001), vol. 445, pp. 151-185.

Benyahia S., 2012, Analysis of Model Parameters Affecting the Pressure Profile in a Circulating Fluidized Bed, AIChE Journal, Volume 58, Issue 2, pp 427-429

Van Deemter and van der Laan, 1961, Momentum and Energy Balances For Dispersed Two-Phase Flow, Journal of Applied Science Research, section A, Vol. 10, pp 102-110

Lu B, Wanga W., Lia J., 2009, Searching for a Mesh-Independent Sub-Grid Model for CFD Simulation of Gas-Solid Riser Flows, Journal of Chemical Engineering Science, Volume 64, Issue 15, pp 3437- 3447

Gidaspow D., 1994, Multiphase Flow and Fluidization: Continuum and Kinetic Theory Descriptions with Applications. Academic Press, Boston, pp 315-316

Hong K., Wang W., Zhou Q., Wang J., Li J., 2012, An EMMS-Based Multi-Fluid Model (EFM) for Heterogeneous Gas-Solid Riser Flows: Part I. Formulation of StructureDependent Conservation Equations, Journal of Chemical Engineering Science, Volume 75, pp 376-389

Hu H.H., Patankar N.A., Zhu, M.Y., 2001, Direct numerical simulations of fluid-solid systems using the arbitrary Lagrangian-Eulerian technique. Journal of Computational Physics, Volume 169, Issue 2, pp 427-462 
Igci Y., Sundaresan S. ,2011, Constitutive Models for Filtered Two-Fluid Models of Fluidized Gas_Particle Flows, Industrial Engineering Chemistry Research, Issue 50, pp 13190-13201

Igci Y., Pannala S., Benyahia S., \& Sundaresan S., 2012, Validation Studies on Filtered Model Equations for Gas-Particle Flows in Risers, Industrial \& Engineering Chemistry Research, Volume 51, Issue 4, pp 2094-2103

Israelachvili J., 1991, Intermolecular and Surface Forces, Academic Press, London, UK, $3^{\text {rd }}$ edition, ISBN 978-0-12-375182-9 (ALK. paper)

Jenkins JT, Zhang C., 2002, Kinetic theory for identical, frictional, nearly elastic spheres, Phys Fluids. Volume 14, pp 1228-1235

Li H., Xia Y., Tung Y., Kwauk M., 1991, Micro-visualization of clusters in a fast fluidized bed, Powder Technology, Volume 66, pp 231-235

Li J., Kwauk M., 1994, Particle-Fluid Two Phase Flow, The Energy Minimization Multiscale Method, Metallurgy Industry Press, Beijing

Li J., Ge W., Wang W., Yang N., Liu X., Wang L., He X., Wang X., Wang J., Kwauk M., 2013, From Multiscale Modeling to Meso-Science, A Chemical Engineering Perspective Principles, Modeling, Simulation, and Application, Springer Heidelberg New York Dordrecht London, Library of Congress Control Number: 2012954048, DOI 10.1007/978-3-642-35189-1

Lu B., Wang W., Li J., 2009, Searching for a mesh-independent sub-grid model for CFD simulation of gas-solid riser flows, Journal of Chemical Engineering Science, Volume 64, Issue 15, pp 3437-3447

McKeen T., Pugsley T., 2003, Simulation and Experimental Validation of a Freely Bubbling Bed of FCC Catalyst, Journal of Powder Technology, Volume 129, Issues 1-3, pp 139-152

Milioli, C. C., Milioli F. E., Holloway W., Agrawal, K., Sundaresan S., 2013, Filtered Two-Fluid Models of Fluidized Gas-Particle Flows: New Constitutive Relations, AIChE Journal, Volume 59, Issues 9, pp 3265-3275,

Pannala S., Syamlal M., O'Brien T. J., 2011, Computational Gas-Solids Flows and Reacting Systems: Theory, Methods and Practice, ISBN-13: 978-1615206513, Engineering Science Reference 
Qi H.Y., Li F., Xi B, You C., 2007. Modeling of Drag with the Eulerian Approach and EMMS Theory for Heterogeneous Dense Gas-Solid Two-Phase Flow, Journal of Chemical Engineering Science, Volume 62, Issue 6, pp 1670-1681

Seville J.P.K., Willett C.D., Knight P.C., 2000, Interparticle Force in Fluidization: a review, J. of Powder Technology, Volume 113, Issue 3, pp 261-268

Syamlal M., Rogers W., O’Brien T.J, 1993, MFIX Documentation; Theory Guide, Technical Note, U.S. Department of Energy, Office of Fossil Energy, Morgantown Energy Technology Center, Morgantown, West Virginia

Syamlal M., Rogers W., O'Brien T.J., 1993, MFIX Documentation: Volume 1, Theory Guide, National Technical Information Service, Springfield, VA, Appendix A

Syamlal M., O’Brien T. J., 2003, Fluid Dynamic Simulation of O3 Decomposition in 3 a Bubbling Fluidized Bed, AIChE Journal, Volume 49, Issue 11, pp 2793-2801

Tenneti S., Garg R., Subramaniam S., 2011, Drag Law for Monodisperse Gas-solid Systems Using Particle-resolved Direct Numerical Simulation of Flow Past Fixed Assemblies of Spheres, International Journal of Multiphase Flow, Volume 37, Issue 9, pp 1072-1092

Tsuji Y., Kawaguchi T., Tanaka T., 1993, Discrete particle simulation of a fluidized bed. Powder Technology, Volume 77, Issue 1, pp 79-87

Van Deemter, J. J., van der Laan, E. T., ,1961, Momentum And Energy Balances for Dispersed Two-Phase Flow, Journal of Applied Sciences Research, Section A, Volume 10, pp 102-108

Wen C. Y., Yu Y. H., 1966, Mechanics of Fluidization, Chemical Engineering Progress Symposium Series, Volume 62, Issue 1, pp 100-111

Wang J., Ge W., Li J., 2008, Eulerian Simulation Of Heterogeneous Gas-Solid Flows in CFB Risers: EMMS-based Sub-Grid Scale Model with a Revised Cluster Description, Journal of Chemical Engineering Science, Volume 63, Issue 6, pp 1553-1571

Wang J., 2009, A Review of Eulerian Simulation of Geldart A Particles in Gas-Fluidized Beds, Journal of Industrial and Engineering Chemistry Research, volume 48, Issue 12, pp 5567-5577

Wang W., Li J., 2007, Simulation of Gas-solid Two-phase Flow by a Multiscale CFD Approach: Extension of the EMMS Model to the Sub-grid Scale Level, Journal of Chemical Engineering Science, Volume 62, Issues 1-2, pp 208-231. 
Xu M., Chen F., Liu X., Ge W., Li J., 2012, Discrete Particle Simulation of Gas-Solid Two-Phase Flows with Multi-scale CPU-GPU Hybrid Computation, Journal of Chemical Engineering, Volumes 207-208, pp 746-757

Xu J., Zhu J.X., 2011, Visualization of particle aggregation and effects of particle properties on cluster characteristics in a CFB riser, Chemical Engineering Journal, Volume 168, Issue 1, pp 376-389

Ye M., van der Hoef M.A., Kuipers J.A.M., 2005a, From Discrete Particle Model To A Continuous Model of Geldart A Particles, Journal of Chemical Engineering Research and Design, Volume 83, Issue 7, pp 833-843

Ye M.A, van der Hoef M.A., Kuipers J.A.M., 2005b, The Effects of Particle and Gas Properties on the fluidization, Journal of Chemical Engineering Science, Volume 60, Issue 16, pp 4567- 4580

Ye M. A., van der Hoef M.A., Kuipers J.A.M, 2008, Two-fluid modeling of Geldart A particles in gas-fluidized beds, Journal of Particuology, Volume 6, Issue 6, pp 540-548

Yang N., Wang W., Ge W., Li J., 2003, CFD Simulation of Concurrent-up Gas-Solid Flow in Circulating Fluidized Beds with Structure-Dependent Drag Coefficient, Journal of Chemical Engineering, Volume 96, Issues 1-3, pp 71-80

Yang N., Wang W., Ge W., Li J., 2004, Simulation of Heterogeneous Structure in a Circulating Fluidized-Bed Riser by Combining the Two-Fluid Model with the EMMS Approach

Zhang D.Z., Vanderheyden W.B., 2002, The Effects of Mesoscopic Structures on the Macroscopic Momentum Equations for Two-Phase Flows. International Journal of Multiphase Flow, Volume 28, Issue 5, pp 805-822 
CHAPTER 4: A CLUSTER-AWARE DRAG MODEL FOR SIMULATION OF PARTICLE-GAS FLOWS INVOLVING COHESIVE INTERPARTICLE FORCES

\subsection{Abstract}

The present paper includes a proposed method for calculation of solid-gas drag coefficient in the Two-Fluid Modeling (TFM) approach using drag coefficients that are obtained from direct numerical simulations of flow around particles. A fluidized bed flow was simulated in the MFXI program in order to test the performance of the proposed method in solid volume fraction of $\varnothing=0.087$. The proposed drag model assumes a correction to the standard drag laws to account for the particle clustering in the regions of the computational domain where particle clusters are predicted by the model. A correction parameter reported by S. Subramaniam (2015, private communication) for particle-resolved direct numerical simulation of freely evolving suspension of FCC particles is used in the present work. The correction parameter is the function of Reynold number of particles and switching to the cluster model is based on a cohesive index. It was found that in fluidization of FCC particles with $d_{p}=5.4 \times 10^{-6} \mathrm{~m}$, Reynolds $\sim 0$ to 60 , and granular temperature 0.046 to $2.728 \mathrm{~m}^{2} / \mathrm{s}^{2}$, an improvement of 60 percent could be obtained using the proposed model in comparison to using the standard drag model.

\subsection{Introduction}

The TFM approach, developed by van Deemter and van der Laan (1961), is known as an economic way of simulating multiphase flows in large-scale fluidized bed risers (Pannala et al., 2011). Formulating the solid and gas as continuous phases is the principle of the 
TFM method. This leads to significant reduction of memory and computational costs as compared to other widely exploited methods, such as the Particle-Resolved Direct Numerical Simulation (Hu et al., 2001 and Nomura and Hughes, 1992), Discrete Element Method (Tsuji et al. 1993 and Mikami et al., 1998), and structure-based methods, such as the Discrete Bubble Model (Bokkers et al. 2006), and the Discrete Cluster Model (Liu et al., 2006 and Zou et al. 2008). One notable drawback of the TFM in MFIX is the absence of cohesive inter-particular forces, such as electrostatic and van der Waals forces between particles. These forces play a major role in fluidization of strongly cohesive particles in Geldart A and C groups by creating heterogeneous structures, called clusters. According to Li et al. (2013), clusters affect the flow significantly by changing the mass and momentum transfers between the gas and solid phases. Many researchers, such as Agrawal et al. (2001), Zhang and Vanderheyden (2002), McKeen and Pugsley (2003), Yang et al. (2003, 2004), Ye et al. (2005a,b, 2008), Qi et al. (2007), Wang et al. (2007), Wang et al. (2008 and 2009), Lu et al. (2009), Igci et al. (2012), and Li et al. (2013), believe that clusters are responsible for significant reduction of the interfacial drag forces between the gas and solid phases. Therefore, dependency of the drag forces on the nature of the attractive interparticle forces plays as important a role as the dependency on two other parameters, i.e., the Reynolds number of the flow around particles and the volume fraction of the solid phase in each computational cell. There have been several attempts to improve the performance of the MFIX-TFM code by introducing more complex drag laws, which can consider the effect of subgrid-scale heterogeneous structures in TFM simulations, such as the filtered models of Igci et al. (2011) and Milioli et al. (2013). However, the constitutive models used in these filtered models were obtained from 
highly resolved simulations of kinetic theory-based TFM simulations in the absence of the cohesive interparticle forces. This gap can be filled by inclusion of cohesive interparticle forces in the MFIX-TFM code, similar to inclusion of van der Waals in the MFIX-DEM code (MFIX-2013 Release Notes). In addition, no study has been found in the literature that has implemented the inclusion of the van der Waals forces in the drag laws within the MFIX-TFM code.

DNS has been widely used in high resolution simulation of gas-particle flows in suspension and fluidized beds by researchers such as Ma et al. (2006), Cho et al. (2005), Xiong et al (2012), and Yin and Sundaresan (2009). Ma et al. (2006) acknowledged the diversity and structural dependence of the drag force on each particle, rather than relying on the entire control volume performed in methods such as TFM. Their analysis, akin to DNS analysis of Xiong et al (2012), proved that the drag force is significantly different on particles in dilute regions compared to grouped particles.

One useful approach in DNS modeling is the analysis of the flow over fixed assemblies of particles, as practiced by Hill et al. (2001a,b), van der Hoef et al. (2005), Beetstra et al. (2007), Yin and Sundaresan (2009), and Tenneti and Subramaniam (2011). This approach increases the accuracy and relevance of the information collected. For example, information about field variables, such as the coefficient of drag, and gas and particle velocities can be obtained. Additionally, various different cluster configurations could be analyzed. Cluster differences include: shape, compactness, orientation of the cluster relative to the fluid, spinning speed of the cluster, and various flow-solid relative 
velocities. A combined particle or cluster resolved DNS analysis coupled with the TFM analysis of the flow could contribute to the improvement of the TFM modeling of the clustering multiphase flow systems. There is also an opportunity for a simulation of the flow on the industrial-scale, using the information obtained in particle or cluster-scale.

Presently, MFIX is a widely known, reliable, and professionally established package for simulation of heat and mass transfer. MFIX accommodates a variety of drag models that can be used in TFM simulation of gas-solid particulate flows. Yet, the direct or indirect addition of models for particle-to-particle, attractive and repulsive forces to the transport equations solved in TFM, or to the available drag laws, is missing. According to Ye et al. (2005 a,b) and Seville et al. (2000), these forces could be formulated as $\vec{F}_{i j}{ }^{(c)}=\left(A R / 6 d_{i j}{ }^{2}\right)$ $\overrightarrow{\mathrm{n}}_{\mathrm{ij}}$, where $\mathrm{F}_{\mathrm{ij}}{ }^{(\mathrm{c})}$ is the cohesive inter-particular force and $\mathrm{A}$ is the Hamaker constant $\left(\approx 10^{-19}\right.$ J) (Israelachvili, 1991), $\mathrm{R}$ is the radius of the mono-dispersed particles, $\mathrm{d}$ is the surface to surface distance between particles and $\vec{n}$ is the normal vector pointing from the center of particles $\mathrm{i}$ to the center of the particle $\mathrm{j}$. Further, they defined a scaling factor, $\varphi=\frac{\left|U_{\min }\right|}{K_{B} T}=\frac{A R}{6 z_{0}} \cdot \frac{1}{K_{B} \Theta_{S}}$, which is the ratio between the interparticle cohesive and destabilizing forces for $\mathrm{d} \leq 100 \mu \mathrm{m}$. In this definition, $\mathrm{K}_{\mathrm{B}}$ is the Boltzmann constant $\left(\mathrm{K}_{\mathrm{B}} \approx\right.$ 1, Ye et al., 2005a), $\mathrm{Z}_{0}$ is the threshold for particles to be considered as clustered $\left(\mathrm{Z}_{0} \approx 4\right.$ $\mathrm{nm}$, Seville et al., 2000) and $\mathrm{d}$ and $\Theta_{\mathrm{s}}$ are the diameter and granular temperature of the solid particles. The derivation of equations governing the particle motion can produce a similar quantifying scaling factor, which can indicate the onset of cluster formation. In this analysis, as compared to the cohesion models available in the MFIX-DEM, the 
scaling factor is an additional factor to be considered for cluster formation, (in addition to the surface to surface particle distances).

Destabilizing forces in the particle-gas systems are mainly due to the particle-to-particle and particle-to-gas interactions. These interactions significantly influence the analysis of particle-gas flows, which has attracted the attention of many researchers, such as Dombrowski and Johns (1963), Gidaspow (1994), Ding and Gidaspow (1994), Cho et al. (2005), Benyahiah (2012), Karimipour and Pugsley (2012), and Syamlal et al.(2003). Special attention has been paid to this parameter in the work of Yet et al. (2005-a). The granular temperature is a measure of the particle fluctuating energy and could be used as a critical parameter to predict the coalescence of particles and break-up of clusters in numerical simulations. MFIX-TFM can solve the transport equation or the algebraic equation, in order to obtain the granular temperature.

In this study, we introduce a cohesive index into the MFIX-TFM code and implement it as a criterion for switching between a Particle-Resolved Direct Numerical-Simulation model, the TGS model, and three existing drag models available in the MFIX code. The rest of the paper is organized as follows. First, the model formulation is presented where the governing equations for the TFM model, the governing equations related to the model of motion of particles leading to our cohesive index and the governing equations of the Gidaspow, Syam-O’Brien, Wen-Yu and TGS drag models were presented. Later, the methodologies for implementing the cohesive index, error calculations and switching between the TGS and other models are presented and followed by the examination of the 
proposed models in numerical simulations for flow in a fluidized bed. Finally, a conclusion is drawn on the effectiveness of the proposed model and the authors' perspective of the future work.

\subsection{Numerical Model}

In the TFM, both the gas and the particulate phases are considered as interpenetrating continuous mediums. Complete derivations of the equations governing the two-fluid model can be found in the work of Gidaspow (1994). Summary of equations governing the TFM are available in Table 3-1 of chapter 3 of this dissertation.

Ha parameter is defined as

$$
\mathrm{Ha}=\frac{\mathrm{A}}{\pi \rho \mathrm{d}_{\mathrm{p}}^{2} \mathrm{~d}_{0} \Theta_{\mathrm{S}}}
$$

In this expression, $\rho$ is the density of the solid particle, $d_{p}$ is the particle mean diameter, $\mathrm{d}_{0}$ is the surface to surface cut-off distance, and $\Theta$ is the granular temperature. The Ha parameter is the ratio of interparticle cohesive force to the destabilizing force, kinetic energy, acting on each particle in the computational domain. In our TFM simulations, the Ha parameter can be obtained for each computational node for the continuous representation of the solid phase. In fact, an increase of this parameter can be related to the increase of clustering chance in the domain. This heterogeneity discourages the use of the standard models in the zones designated by clustering, since standard modes are appropriate for non-clustered particles. Therefore, it is appropriate to switch to a nonuniform drag model where large values of Ha are predicted by the numerical models. This idea is conceptualized in a proposed drag model, as illustrated in Table 4-1. 
Table 4-1 Proposed hybrid drag model scheme using the cohesive index for MFIXTFM simulations.

\begin{tabular}{|c|c|c|}
\hline New drag model & Criteria & Definition \\
\hline Use $\mathrm{F}_{\mathrm{cl}}{ }^{*}$ & Ha $>$ Ha-THS & $\mathrm{F}_{\mathrm{cl}}=\mathrm{Fu} * \mathrm{~g}(\mathrm{Re})$ \\
\hline Use $\mathrm{F}_{\mathrm{u}}{ }^{* *}$ & $\mathrm{Ha} \leq \mathrm{Ha}-\mathrm{THS}$ & $\mathrm{Fu}=\mathrm{F}_{\text {(Syam-O’Brien) }}$ \\
\hline
\end{tabular}

One possible approach to define a modified from of the drag based on clustering phenomenon is to compare the drag coefficients obtained from PR-DNS simulation of flow around particles in a homogenous configuration and particle/clusters in a nonhomogenous configuration. In this perspective, $\mathrm{F}_{\mathrm{cl}}$ can be defined as a modified drag law as $\mathrm{F}_{\mathrm{cl}}=\mathrm{g} * \mathrm{~F}_{\mathrm{u}}$, where $\mathrm{g}$ is a modification factor that must be adjusted dynamically to close the set of equations. To perform the PR-DNS simulations, one possibility is to use realizations introduced by Tenneti and Subramaniam (2013). In the framework of TFM, it is acceptable to use fluid flow information from PR-DNS of an assembly which has the same non-dimensional parameters, such as solid volume fraction, Reynolds number, and cohesion index. However, availability of and accessibility to PR-DNS information sets a limit on the amount of information that can be used in this procedure. Thus, a major contribution can involve conduct of tedious and expensive simulations with different ranges of non-dimensional parameter, e.g., $\varepsilon_{s}, \Phi$, Re, etc., in PR-DNS and grant of access to the results is appreciable. Such valuable information was provided by Mehrabadi and Subramaniam (2015, private communication) for $\Phi=0.087$ and $\operatorname{Re} \sim 0$ to 60 and $\mathrm{Ha} \sim$ 0.00063 to 0.0377 for FCC particles with mean diameter of $54 \mu \mathrm{m}$. These researchers quantified the $\mathrm{F}_{\mathrm{cl}} / \mathrm{F}_{\mathrm{u}}$ ration as a function of Reynolds number represented by Eq. (11). 


$$
\left(\frac{F_{c l}}{F_{u}}\right)_{\mid \Phi=0.087}=g(R e)=\frac{a \times b+c e_{m}^{d}}{b+\operatorname{Re}_{m}^{d}}
$$

In this definition, $a=0.5378, b=305.9554, c=0.8045$, and $d=1.6292$. For the present analysis, we adopt the above definition to be used for the parameter g used in Eq. (11).

\subsection{Simulation Methodology}

The performance of the constituent and hybrid drag models were evaluated initially for a 20-second simulation of the flow in a circulating fluidized used in the experiment of $\mathrm{Li}$ and Kwauk (1994). Dimensions and boundary conditions of the computational domain are displayed in Figure 4-1. Readers can refer to Chapter 2 of this dissertation for more details and choice of computational grid size. We obtained permission from Li and Kwauk (1994) and Hong et al. (2012) to use their experimental and numerical data in our analysis, respectively. In addition, the results obtained from Abbasi et al. (2015) and available in Chapter 2 of this dissertation were included in the comparisons. Abbasi et al. (2015) showed that application of a hybrid drag model (AGDSM1) in the form of (Syam-O’Brien $\rightleftharpoons$ TGS) could substantially increase the accuracy of the simulation results. They associated this modification to the corrections applied to the drag estimates in the dense areas of the domain, where most of clustering instances were expected. In the present study, similar to the work of Hong et al. (2012) and Abbasi et al. (2015), we used laminar flow assumption for the air and considered the transport equation of the granular temperature for fluctuations in the solid phase. Details of the simulation settings are available in the work of Abbasi et al. (2015) and Chapter 2 of the present dissertation. 

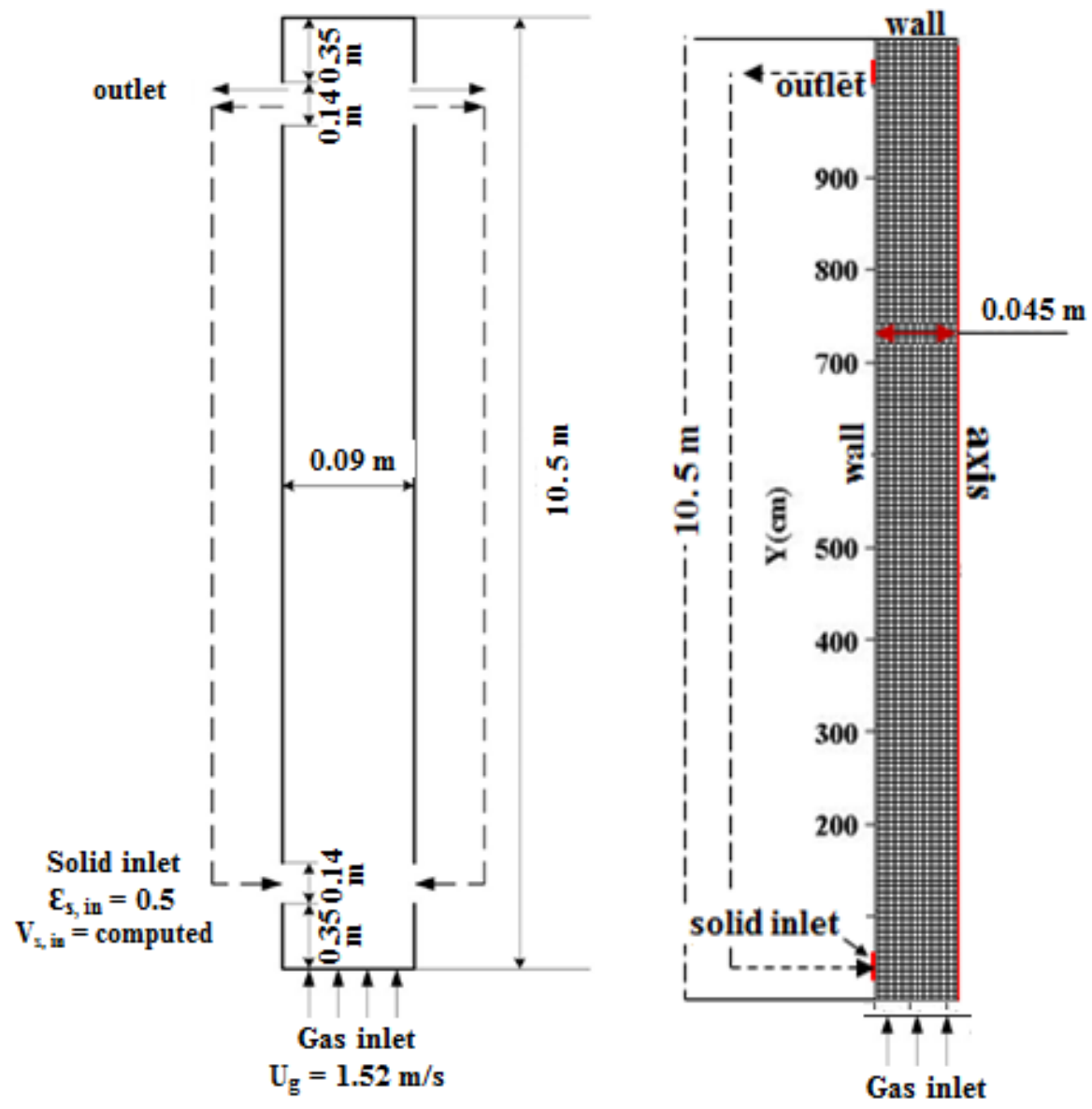

Figure 4-1 Computational domain and boundary conditions used for MFIX-TFM fluidized bed flow simulations

Thresholding for solid volume fraction and granular temperature (i.e., no switching operations if $\varepsilon_{\mathrm{s}} \geq 0.999$ and $\Theta \leq 1 \times 10^{-16}$ ) was performed in accord to Abbasi et al. (2015). It was found necessary to impose these restrictions in the code to avoid the possibility of singularity in Ha calculations in extremely dilute regions of the domain, or for cells with extremely small values of granular temperature. In addition, The Ha-THS parameter was examined in a wide range for all three proposed versions of the drag model in order to find the optimum value. 
Computational results obtained in the last 100 time steps were time and space-averaged on each cross section along the riser of the fluidized bed. The profile of the solid volume fraction was plotted against the available experimental data points and results published by Abbasi et. al. (2015) for the most similar hybrid model (AGDSM-1). A computer script was used to interpolate the numerical results for specific heights of the riser, where data points from the experiment of $\mathrm{Li}$ and Kwauk (1994) were available. Then, the maximum and accumulative deviation of the numerical data points from the corresponding experimental data points along with an average absolute percentage deviation (AAPD) value were calculated by Eq. (12-14), respectively.

$$
\begin{gathered}
\operatorname{Err}_{\text {max }}=\operatorname{Max}\left(\left|f_{\text {exp. }}(\lambda)-f_{\text {sim. }}(\lambda)\right|\right) \\
\operatorname{Err}_{\text {accum. }}=\sum_{\lambda=1}^{N}\left(\left|f_{\text {exp. }}(\lambda)-f_{\text {sim. }}(\lambda)\right|\right) \\
\text { AAPD }=\operatorname{Err}_{\text {avg. }}(\%)=\frac{100}{N} \times \sum_{\lambda=1}^{N}\left(\left|f_{\text {exp. }}(\lambda)-f_{\text {sim. }}(\lambda)\right|\right) / f_{\text {exp. }}(\lambda)
\end{gathered}
$$

In these equations, $\mathrm{f}_{\text {sim }}, \mathrm{f}_{\mathrm{exp}}, \lambda$, and $\mathrm{N}$ are numerical and experimental data point values, index, and number of the measurement locations along the riser of the experimental facility, respectively. Later, a polynomial function was fitted to the experimental data points in order to create a profile with a significantly greater number of points for our further error analysis. Therefore, it was possible to calculate the target parameters, such as the correlation values between the numerical and experimental profiles, i.e., $\mathrm{R}^{2}$, and the AAPD, using 150 points along the riser. Equation (15) shows the expression used for calculation of the $\mathrm{R}^{2}$ value, which is the overall comparison between the simulation 
results and the experimental data, for both of the constituent (i.e., Syam-O’Brien) and the proposed hybrid drag model.

$$
\left(\mathrm{R}^{2}\right)_{\text {model }}=1-\frac{\sum_{\mathrm{i}=1}^{\mathrm{N}}\left(\mathrm{Y}_{\mathrm{i}, \varepsilon_{S}}-\frac{1}{\mathrm{~N}} \sum_{\mathrm{i}=1}^{\mathrm{N}} \mathrm{Y}_{\mathrm{i}, \varepsilon_{S}}\right)^{2}}{\sum_{\mathrm{i}=1}^{\mathrm{N}}\left(\mathrm{Y}_{\mathrm{i}, \varepsilon_{S}}-\mathrm{f}_{\mathrm{i}, \varepsilon_{S}}\right)^{2}}
$$

In this definition $\mathrm{N}, \mathrm{Y}_{\mathrm{i}, \varepsilon s}$, and $\mathrm{f}_{\mathrm{i}, \varepsilon s}$ indicate the total number of data points, $\varepsilon_{\mathrm{s}}$ values on the polynomial fit, and the $\varepsilon_{\mathrm{S}}$ values on numerical profile of each drag model, respectively.

Later, improvement to the constituent model was calculated by comparing the Errmax, AAPD, and $\mathrm{R}^{2}$ values before and after using the model in the proposed hybrid model. Equations (16-18) show the expressions used for calculation of these improvements.

$$
\begin{aligned}
& \text { imp.Err } \max =100 \times \frac{\left.\left|\left(\operatorname{Err}_{\max }\right)_{\text {SY_O'B }_{1}}-\left(\operatorname{Err}_{\text {max }}\right)_{\text {AGDSM1 }}\right|\right)}{\left(\text { Err }_{\text {max }}\right)_{\text {SY_O'B }}} \\
& \text { imp.AAPD }=100 \times \frac{\mid(\mathrm{AAPD})_{\text {constituent }}-(\text { AAPD })_{\text {hybrid }} \mid}{(\text { AAPD })_{\text {constituent }}} \\
& \text { imp_R } R^{2}=100 \times \frac{\left.\left|\left(\mathrm{R}^{2}\right)_{\text {constituent }}-\left(\mathrm{R}^{2}\right)_{\text {hybrid }}\right|\right)}{\left(\mathrm{R}^{2}\right)_{\text {constituent }}}
\end{aligned}
$$

\subsection{Results and Discussion}

Time and area averaged profiles of solid concentration obtained from the proposed hybrid drag model using different values of Ha-THS are shown in Figure 4-2. Results show the sensitivity of the modeling to variation of Ha-THS. Here, we gradually reduced the 
threshold values from 0.1 to $1 \times 10^{-10}$ in order to engage more computational cells in our switching operations. Hereafter, we frequently use the terms dilute and dense regions of the fluidization column for height $>470 \mathrm{~cm}$ and height $<470 \mathrm{~cm}$, respectively. These regions were recognized by the inflation point of the experimental profile occurring at the height $=496 \mathrm{~cm}$ (empty-circle symbols). In addition, the term constituent model is used frequently to refer to the Syam-O'Brien drag model versus the hybrid term which refers to the proposed Syam-O’Brien $\rightleftharpoons \mathrm{g} *$ Syam-O’Brien model.

Comparison of the profiles in Figure 4-2-a shows that the threshold value of Ha-THS = 0.1 has resulted in small deviations from the results originally obtained from the SyamO'brien drag model. Further decrease of the Ha-THS to 0.01 and 0.001 resulted in significantly better prediction of the solid distribution along the fluidization column in Figure 4-2-a and Figure 4-2-b, respectively. An important observation is the excessive solid concentration in regions around the solid inlet valve $(35 \mathrm{~cm} \leq$ height $\leq 50 \mathrm{~cm})$ for Ha-THS to 0.01. Excessive under-prediction of drag forces in the radial direction can account for higher solid axial velocity and over packing of solids in this region. However, the location of the inflation point of the profile is in reasonable agreement with the one for the experimental profile. In the case Ha-THS $=0.001$, the inflation point has move to a higher location, and further reduction the threshold resulted in creation of a secondary inflation point on the profile of Ha-THS $=1 \times 10^{-4}$ and THS $=1 \times 10^{-5}$ around height $=650$ $\mathrm{cm}$ and height $=850 \mathrm{~cm}$, respectively. However, the location of the primary inflation point is in good agreement with the experimental data for Ha-THS $=1 \times 10^{-4}$ and THS $=$ $1 \times 10^{-5}$ and identical results were obtained in the dense regions with these profiles. Figure 
4-2-c shows that further decrease of the threshold resulted in excellent predictions in the lower half of the fluidization column (dense regions) and the location of the primary inflation point, i.e., height $=470 \mathrm{~cm}$, matches the one from the experimental data. However, the prediction is poor in the dilute regions for all of the profiles in Figure 4-2-c, and additional inflation points were created in the dilute regions. This growth of over predictions with reduction of the Ha-THS, which in fact increases the sensitivity of the model to the smallest amount of cohesion forces, can be justified by the over prediction of drag coefficient by the proposed hybrid model in dilute regions. This is in accord with the under prediction of standard drag models due to under estimation of drag forces. Here with all the observations considered, the authors would like to bring this fact to the attention that all the profiles in Figure 4-2(a-c) have one thing in common; improvement of predictions in dense regions of the fluidization column in comparison to the original profile of the constituent model. Another observation in Figure 4-2-c is that beyond HaTHS $=1 \times 10^{-6}$, very similar or identical results were obtained for all profiles. This fact indicates that there are threshold values below which modeling cannot be further improved in the dense regions of the fluidized bed. 


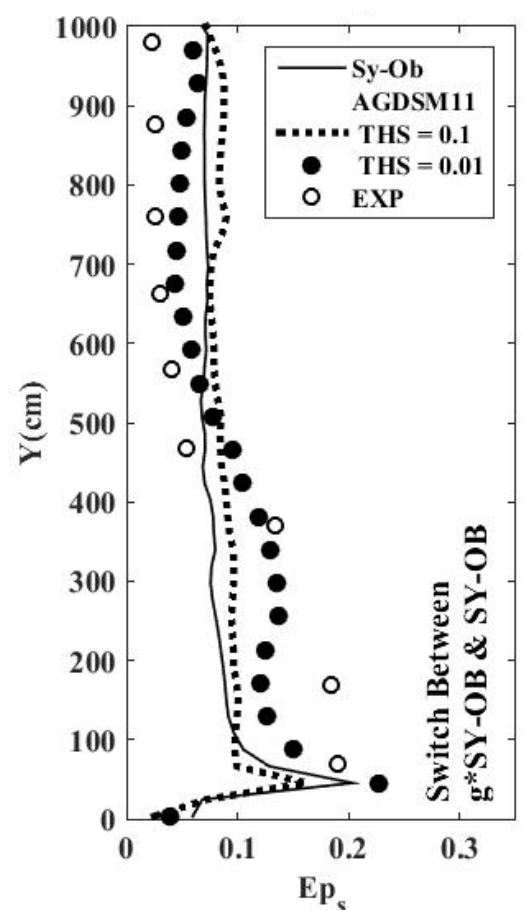

(a)

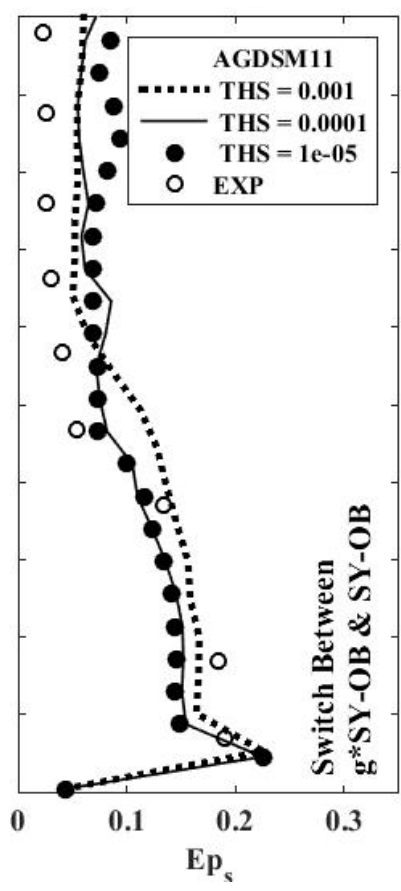

(b)

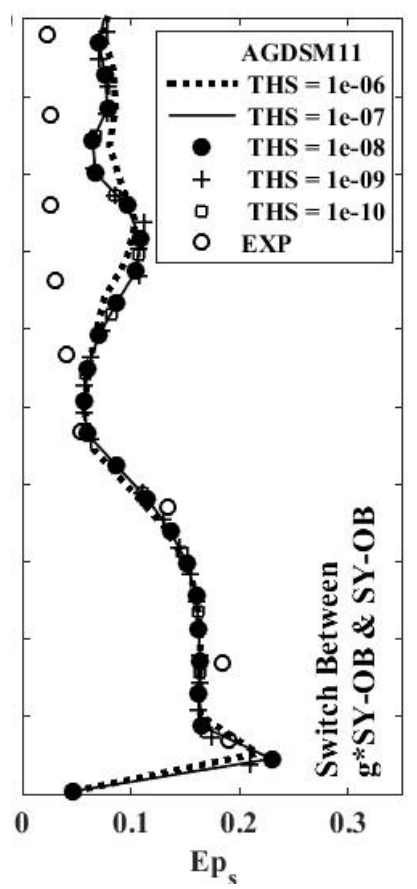

(c)

Figure 4-2 Profile of solid volume fraction along the riser obtained by MFIX_TFM simulations for different values of Ha-THS, experimental data by Li and Kwauk (1994)

A quantitative comparison of the results for all values of the Ha-THS in the range of $\left[1 \times 10^{-10}\right.$ to 0.1$]$ is available in Figure $4-3$. This figure shows comparisons of different error criteria, such as accumulative, maximum, and AAPD errors along with the correlation value, $\mathrm{R}^{2}$. We performed the analysis one time for the entire domain, as shown by solid black lines, and one time for the lower half part of the domain, as shown by dotted blue lines. The analysis is for the entire domain, and the lower half (dense regions) of the domain shown in dotted blue lines. The purpose of including a separate analysis for the dense region is to isolate the error analysis of the dense regime from the influences of the dilute regions. In addition, the motivation for this separate analysis was the tendency of the hybrid model to improve the original profile (the Syam-O'Brien) in the dense regions, where chances of cluster formation are relatively higher than the dilute 
regions, for all values of the Ha-THS. We have also included all errors and $\mathrm{R}^{2}$ values of the Syam-O'Brien drag model against the best values obtained on the solid lines in Figure 4-3 (a-d).

Comparison of the data points for Ha-THS $\leq 0.01$ on solid lines in Figure 4-3 shows a significant drop of the error values from the error values associated with the constituent model. In the case of the correlation value in Figure 4-3, significantly better $\mathrm{R}^{2}$ values were obtained for all threshold values in comparison to the constituent model. However, results show increase of error values toward smaller Ha-THS values after early improvements. The same fact is observable in Figure 4-3, where after an increase of $R^{2}$ values, $R^{2}$ values started to decrease at Ha-THS $=1 \times 10^{-4}$ and reached steady values at Ha-THS $=1 \times 10^{-7}$. In addition, minimum of error values on the solid lines occur at different Ha-THS. For example, minimum of the quantities, such as maximum, accumulated, and average relative errors (AAPD), occurred at $1 \times 10^{-4}, 1 \times 10^{-3}$, and $1 \times 10^{-2}$, respectively. Such discrepancies alongside with local behaviors of the model in terms of improving or weakening the agreement of numerical results with the experimental data, can be related to model parameter (g) which is a function of Reynolds number only for a fix value of $\Phi$ and needs to be tuned for a range of values of solid volume fraction in order to reach a consistent behavior the entire computational domain. Based on these predictions, different interprations of the best threshold value may emerge. For instance, one may claim that the best result was obtained by setting the Ha-THS to $1 \times 10^{-3}$, for the lowest value of summation error, second lowest value of AAPD and second highest value of fit correlation $\left(\mathrm{R}^{2}\right)$. 
In the next step, the analysis is focused on the common fact with all the profiles in Figure 4-3. As mentioned earlier, all profiles in Figure 4-2 show improved predictions of solid concentration in dense regions of the fluidization column. Here, all dotted blue lines in Figure 4-3 well demonstrate this fact by showing lower values of error and higher values of $\mathrm{R}^{2}$ for all values of Ha-THS in Figure 4-3 (a-c) and Figure 4-3-d, respectively. Based on these predictions, error values decrease and $\mathrm{R}^{2}$ value increases by reduction of the threshold values and one can claim that the best agreements with the experimental data were obtained with Ha-THS $=1 \times 10^{-7}$. 


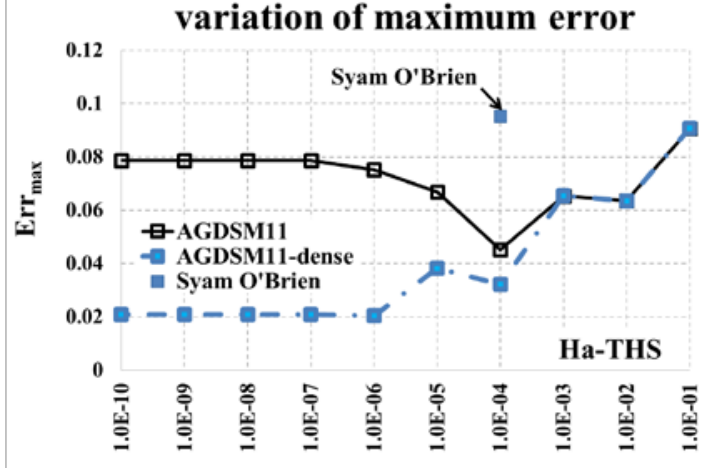

(a)

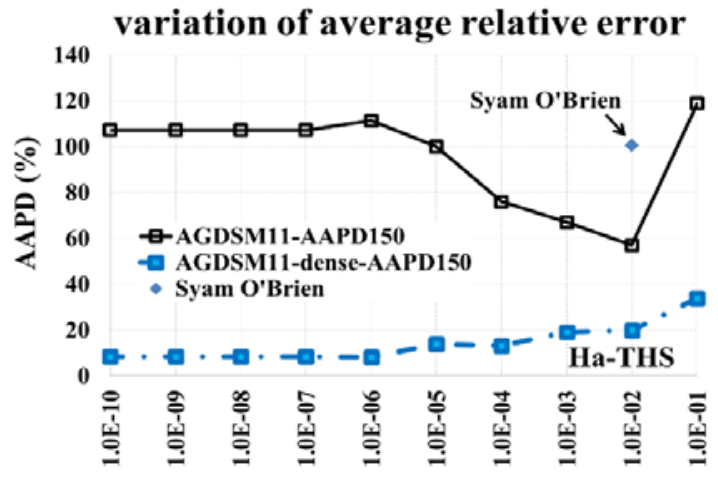

(c)

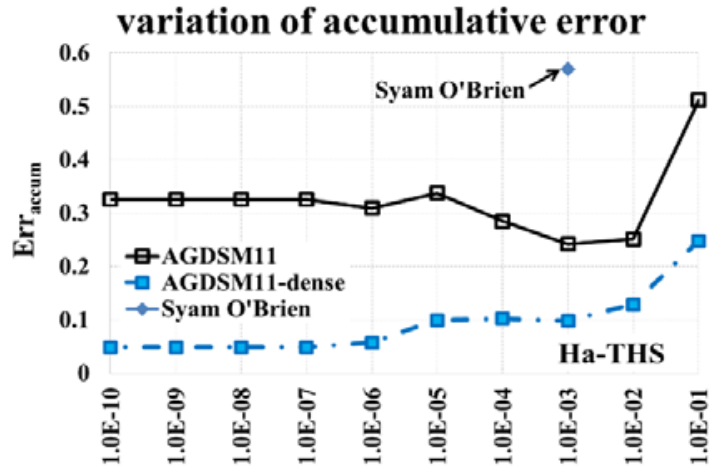

(b)

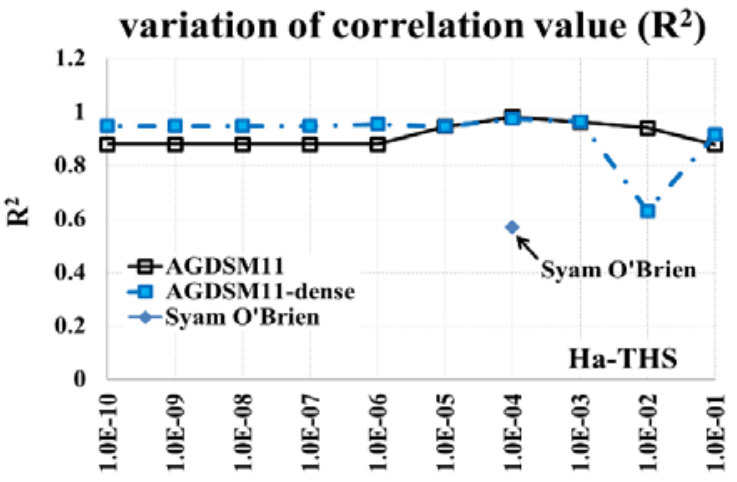

(d)

Figure 4-3 Error in TFM simulation for the proposed cluster drag model, maximum , accumulative, AADP, and fitness correlation

In the end, we paid special attention to a comparison between the best result obtained by Abbasi et al. (2015-a) for the hybrid model AGDSM1 (Syam-O’Brien $\rightleftharpoons$ TGS, Ha-THS = $1 \times 10^{-10}$ ) and the nominated results obtained by the hybrid model AGDSM1 (Syam-O’Brien $\rightleftharpoons \mathrm{g}^{*}$ Syam-O’Brien, Ha-THS $\left.=1 \times 10^{-10}\right)$. Henceforward, the term cluster-aware will be used for the AGDSM11 model. The purpose of this side-by-side comparison is to measure how different a cluster-aware drag model and a non-cluster based drag model, which are both obtained from particle resolved direct numerical simulations, could perform for a similar flow problem. Qualitative and Quantitative comparisons are illustrated in Figure 4-4 and Table 4-2, respectively. As Figure 4-4 
shows, all profiles are similar in regards to the location of the primary inflation point. The secondary inflation points are higher for the AGDSM11 versions and as discussed earlier, this location moved higher for this model with decrease of the threshold. In general, with small deviations in the higher section of the column, predications with Ha-THS $=1 \times 10^{-}$ ${ }^{4}$ (AGDSM11) and Ha-THS $=1 \times 10^{-10}$ (AGDSM1) are close in dilute regions, and better results were obtained by the first model in the dense regions. In addition, profiles have similar inflation points at the height of $650 \mathrm{~cm}$.

Comparison between the AGDSM1 and the cluster-aware models with the same threshold value in Figure 4-4, i.e., Ha-THS $=1 \times 10^{-10}$, reveals a significant difference. In fact, the cluster-aware model shows significantly better results in the dense regions of the fluidization column, but overestimates the solid concentration in the dilute regions more. To quantify the differences between models and improvements of the errors, quantities expressed via equations (12-18) are calculated for both the entire computational domain and dense regions of the domain (Table 4-2). This table shows that relative error in the dense regions of the computational domain, i.e., height $<470 \mathrm{~cm}$, is below 9 percent for the cluster-aware model and is about 30 percent for the AGDSM1 model. In the case of other quantities, Table 4-2 shows better values for the cluster-aware model in the dense regions. However, as far as the entire computational domain is considered, smaller improvements were obtained by the cluster-aware model in comparison to the AGDSM1 model. It is believed that limitation on the model performance lies on the fact that the $\mathrm{g}$ is only a function of Reynold number in this study. Investigations using more sophisticated forms of the $\mathrm{g}$ function, i.e., $\mathrm{g}(\mathrm{Re}, \Phi)$ or $\mathrm{g}(\mathrm{Re}, \Phi, \mathrm{Ha})$ are necessary for better predictions 
in dilute regions where there are still chances of cluster formation. Therefore, additional DNS studies in the range of $0 \leq \Phi \leq 0.2$ can pave the way to finding more precise and sophisticated forms of $g$ functions.

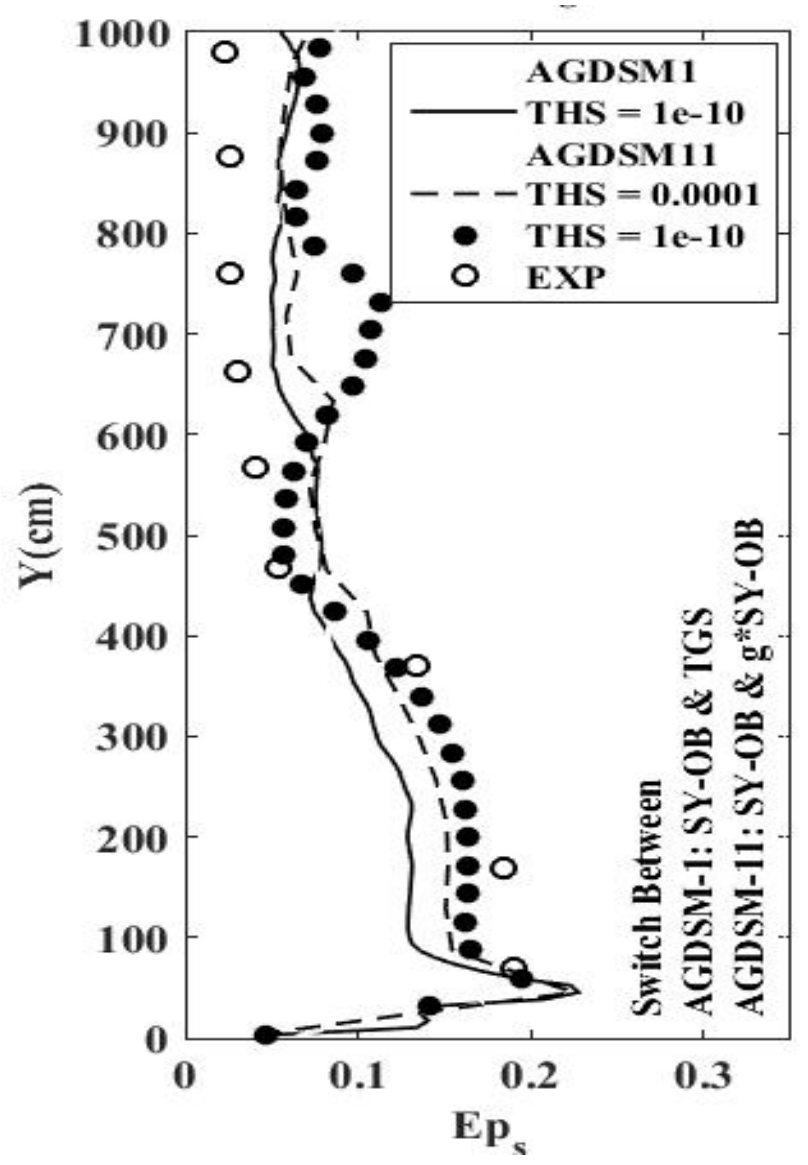

Figure 4-4 Comparison of performance of hybrid models, Cluster-aware drag model proposed in this work in dashed line (Ha-THS $=1 \times 10^{-4}$ ) and empty circles (Ha-THS $=$ $1 \times 10^{-10}$ ) and AGDSM1 from Abbasi et al. (2015) for Ha-THS = $1 \times 10^{-10}$ 
Table 4-2 Error calculations and best improvements for the AGDSM11 model

\begin{tabular}{cccccccc}
\hline $\begin{array}{c}\text { Simulation } \\
\text { case }\end{array}$ & Err. max & $\begin{array}{c}\text { AAPD- } \\
9^{\mathrm{a}} \\
(\%)\end{array}$ & $\begin{array}{c}\text { AAPD- } \\
150^{\mathrm{b}} \\
(\%)\end{array}$ & $\mathrm{R}^{2}$ & $\begin{array}{c}\text { imp.Err } \\
(\%)\end{array}$ & $\begin{array}{c}\text { imp_AAPD } \\
(\%)\end{array}$ & $\begin{array}{c}\text { imp_R } \\
(\%)\end{array}$ \\
\hline $\begin{array}{c}\text { Hong et al. } \\
\text { (2012) }\end{array}$ & 0.077 & 166.1 & 138.5 & 0.56 & - & - & - \\
$\begin{array}{c}\text { Syam-O'Brien } \\
\text { Entire }\end{array}$ & 0.095 & 106.2 & 100.7 & 0.79 & - & - & - \\
dense & 0.095 & 40.1 & 41.4 & 74.2 & - & - & - \\
AGDSM-1 & & & & & & & \\
Entire & 0.053 & 74.5 & 68.4 & 0.97 & 44.5 & 32.0 & 23.2 \\
dense & 0.053 & 29.2 & 23.4 & 0.95 & 44.2 & 43.3 & 27.9 \\
AGDSM-11 & & & & & & & \\
Entire & 0.079 & 119.3 & 107.2 & 0.88 & 16.8 & - & 11.3 \\
dense & 0.021 & 8.9 & 8.6 & 0.95 & 77.9 & 79.2 & 27.8 \\
\hline
\end{tabular}

a: 9-point absolute average percent deviation

b: absolute average percent deviation using the $4^{\text {th }}$ order polynomial fit (150 point)

c : Ha-THS $=1 \times 10^{-10}$

$\mathrm{d}:$ Ha-THS $=1 \times 10^{-10}$

\subsection{Conclusion}

In this chapter, a cluster-aware drag model for simulation of fluidization flow of cohesive particles was presented. The model was tested for FCC material using the Two-Fluid Modeling approach. The performance of this hybrid model proved to be superior over its constituent standard drag model, i.e., Syam-O’Brien, for a broad range of the model's cohesion parameter, i.e, Ha $\geq 1 \times 10^{-10}$. Larger values of the cohesion threshold (Ha-THS) proved to be more effective once the entire computational domain was considered for error evaluation. It was observed that reduction of the threshold did not improve the solid concentration profile in the upper half section of the bed and in some cases, weakened the agreement with the experimental data. However, by isolation of the dilute regions of the domain (upper half section of the bed), it was proven that the model responded better to 
successive reduction of threshold values in the dense regions, where higher clustering chances presented. Sensitivity of the model to the smallest cohesion forces was increased in both the dilute and dense regions of the domain by reduction of the cohesion threshold. The minimal effectiveness of the model was found in the upper bound of the threshold, Ha-THS $=0.1$, where almost the same result of the original constituent model was recovered. In addition, the model responded equally to the threshold values less than $10^{-7}$. Results obtained in this paper support the functionality of the concept of a cohesive index for clustering prediction and suggest a direct incorporation of the granular temperature or the cohesive index in the formulation of a future advanced hybrid drag model. In addition, utilization of a clustering modification factor which is a function of both Reynolds number and solid volume fraction is strongly recommended. Hereby, the authors would like to acknowledge the Department of Energy's National Energy Technology Laboratory for supporting this work. The authors would also like to acknowledge the Applied Research Center of the Florida International University, and collaborators from the Iowa State University, M. Mehrabadi and S. Subramaniam, for their outstanding help and support.

\subsection{References}

Abbasi Baharanchi A., Gokaltun S., Dulikravich G.S., 2015, Performance Improvement of Existing Drag Models in Two-Fluid Modeling of Gas-Solid Flows Using a PR-DNS Based Drag Model, Journal of Powder Technology, Volume 220, pp 63-69

Agrawal K., Loezos P.N., Syamlal M., Sundaresan S., 2001, The role of meso-scale structures in rapid gas solid flows, Journal of Fluid Mech. (2001), vol. 445, pp. 151-185. 
Beetstra R., Van Der Hoef M.A., Kuipers J.A.M, 2007, Drag Force of Intermediate Reynolds Number Flow Past Mono- and Bidisperse Arrays of Spheres, AIChEJ Journal, Volume 53, Issue 2, pp 489-501

Benyahia S., 2012, Analysis of Model Parameters Affecting the Pressure Profile in a Circulating Fluidized Bed, AIChE Journal, Volume 58, Issue 2, pp 427-429

Bokkers G. A., Laverman, J. A.,van Sint Annaland, M., Kuipers, J. A. M., 2006, Modelling of large-scale dense gas-solid bubbling fluidized beds using a novel discrete bubble model, Journal of Chemical Engineering Science, Volume 61, Issue 17, pp 5590-5602

Cho S.H., Choi H.G., Yoo J.Y. 2005, Direct Numerical Simulation of Fluid Flow Laden with Many Particles, International Journal of Multiphase Flow, Volume 31, Issue 4, pp 435-451

Ding J., Gidaspow D., 1990, A Bubbling Fluidisation Model Using Theory of Granular Flow, AIChEJ Journal, Vol. 36, Issue 4, pp 523-538

Dombrowski N., Johns W. R., 1963, The Aerodynamic Instability and Disintegration of Viscous Liquid Sheets, Journal of Chemical Engineering Science, Vol. 18, Issue 3, pp 203-214

Gidaspow D., 1994, Multiphase Flow and Fluidization: Continuum and Kinetic Theory Descriptions with Applications. Academic Press, Boston, pp 315-316

Hill, R.J., Koch D.L., Ladd, A.J.C., 2001a, The First Effects of Fluid Inertia on Flows in Ordered and Random Arrays of Spheres. Journal of Fluid Mechanics, Volume 448, pp 213-241

Hill R.J., Koch D.L., Ladd A.J.C., 2001b, Moderate-Reynolds-Number Flows in Ordered and Random Arrays of Spheres, J. of Fluid Mechanics, Volume 448, pp 243-278

Hong K., Wang W., Zhou Q., Wang J., Li J., 2012, An EMMS-Based Multi-Fluid Model (EFM) for Heterogeneous Gas-Solid Riser Flows: Part I. Formulation of StructureDependent Conservation Equations, Journal of Chemical Engineering Science, Volume 75, pp 376-389

Hu H.H., Patankar N.A., Zhu, M.Y., 2001, Direct numerical simulations of fluid-solid systems using the arbitrary Lagrangian-Eulerian technique. Journal of Computational Physics, Volume 169, Issue 2, pp 427-462 
Igci Y., Sundaresan S. ,2011, Constitutive Models for Filtered Two-Fluid Models of Fluidized Gas_Particle Flows, Industrial Engineering Chemistry Research, Issue 50, pp 13190-13201

Igci Y., Pannala S., Benyahia S., \& Sundaresan S. ,2012, Validation Studies on Filtered Model Equations for Gas-Particle Flows in Risers, Industrial \& Engineering Chemistry Research, Volume 51, Issue 4, pp 2094-2103

Israelachvili J., 1991, Intermolecular and Surface Forces, Academic Press, London, UK, $3^{\text {rd }}$ edition, ISBN 978-0-12-375182-9 (ALK. paper)

Karimipour S., Pugsley T., 2012, Application of the Particle in Cell Approach for the Simulation of Bubbling Fluidized Beds of Geldart A Particles, Journal of Powder Technology, Volume 220, pp 63-69

Li J., Kwauk M., 1994, Particle-Fluid Two Phase Flow, The Energy Minimization Multiscale Method, Metallurgy Industry Press, Beijing

Li J., Ge W., Wang W., Yang N., Liu X., Wang L., He X., Wang X., Wang J., Kwauk M., 2013, From Multiscale Modeling to Meso-Science, A Chemical Engineering Perspective Principles, Modeling, Simulation, and Application, Springer Heidelberg New York Dordrecht London, Library of Congress Control Number: 2012954048, DOI 10.1007/978-3-642-35189-1

Liu X., Xu X., Zhang W., 2006, Numerical simulation of dense particle gas two-phase flow using the minimal potential energy principle. Journal of University Science and Technology Beijing, Mineral, Metallurgy, Material, Volume 13, Issue 4, pp 301-307.

Lu B., Wang W., Li J., 2009, Searching for a mesh-independent sub-grid model for CFD simulation of gas-solid riser flows, Journal of Chemical Engineering Science, Volume 64, Issue 15, pp 3437-3447

Ma J., Ge, W., Wang X., Wang, J. Li J. 2006, High-Resolution Simulation Of Gas-Solid Suspension Using Macro-Scale Particle Methods, Journal of Chemical Engineering Science, Volume 61, Issue 21, pp 7096 -7106

McKeen T., Pugsley T., 2003, Simulation and Experimental Validation of a Freely Bubbling Bed of FCC Catalyst, Journal of Powder Technology, Volume 129, Issues 1-3, pp 139-152

Mikami, T., Kamiya, H., \& Horio, M., 1998, Numerical simulation of cohesive powder behaviour in a fluidized bed, Chemical Engineering Science, Volume 53, Issue 10, pp 1927-1940 
Milioli, C. C., Milioli F. E., Holloway W., Agrawal, K., Sundaresan S., 2013, Filtered Two-Fluid Models of Fluidized Gas-Particle Flows: New Constitutive Relations, AIChE Journal, Volume 59, Issues 9, pp 3265-3275,

Nomura, T., Hughes, T.J.R., 1992. An arbitrary Lagrangian-Eulerian finite element method for interaction of fluid and a rigid body. Journal of Computer Methods in Applied Mechanics and Engineering, Volume 95, Issue 1, pp 115-138

Pannala S., Syamlal M., O'Brien T. J., 2011, Computational Gas-Solids Flows and Reacting Systems: Theory, Methods and Practice, ISBN-13: 978-1615206513, Engineering Science Reference

Qi H.Y., Li F., Xi B, You C., 2007. Modeling of Drag with the Eulerian Approach and EMMS Theory for Heterogeneous Dense Gas-Solid Two-Phase Flow, Journal of Chemical Engineering Science, Volume 62, Issue 6, pp 1670-1681

Seville J.P.K., Willett C.D., Knight P.C., 2000, Interparticle Force in Fluidization: a review, J. of Powder Technology, Volume 113, Issue 3, pp 261-268

Syamlal M., O’Brien T. J., 2003, Fluid Dynamic Simulation of O3 Decomposition in 3 a Bubbling Fluidized Bed, AIChE Journal, Volume 49, Issue 11, pp 2793-2801

Tenneti S., Garg R., Subramaniam S., 2011, Drag Law for Monodisperse Gas-solid Systems Using Particle-resolved Direct Numerical Simulation of Flow Past Fixed Assemblies of Spheres, International Journal of Multiphase Flow, Volume 37, Issue 9, pp 1072-1092

Tenneti S., Subramaniam S., 2013, Particle-Resolved Direct Numerical Simulation for Gas-Solid Flow Model Development, Annual Review of Fluid Mechanics, Volume 46, pp 199-230

Tsuji Y., Kawaguchi T., Tanaka T., 1993, Discrete particle simulation of a fluidized bed. Powder Technology, Volume 77, Issue 1, pp 79-87

van Deemter and van der Laan, 1961, Momentum and Energy Balances For Dispersed Two-Phase Flow, Journal of Applied Science Research, section A, Vol. 10, pp 102-110

van der Hoef M.A., Beetstra R., Kuipers J.A.M., 2005. Lattice-Boltzmann Simulations of Low-Reynolds-Number Flow Past Mono- and Bidisperse Arrays of Sphere: Results for the Permeability and Drag Force, Journal of Fluid Mechanics, Volume 528, pp 233-254 
Wang J., 2009, A Review of Eulerian Simulation of Geldart A Particles in Gas-Fluidized Beds, Journal of Industrial and Engineering Chemistry Research, volume 48, Issue 12, pp 5567-5577

Xion Q., Li B., Zhou G., Fang X., Xu J., Wang J., He X., Wang X., Wang L. , Ge W., Li J., 2012, Large-scale DNS of Gas-Solid Flows on Mole-8.5, Journal of Chemical Engineering Science, Volume 71, pp 422-430

Ye M., van der Hoef M.A., Kuipers J.A.M., 2005a, From Discrete Particle Model To A Continuous Model of Geldart A Particles, Journal of Chemical Engineering Research and Design, Volume 83, Issue 7, pp 833-843

Ye M.A, van der Hoef M.A., Kuipers J.A.M., 2005b, The Effects of Particle and Gas Properties on the fluidization, Journal of Chemical Engineering Science, Volume 60, Issue 16, pp 4567- 4580

Ye M. A., van der Hoef M.A., Kuipers J.A.M, 2008, Two-fluid modeling of Geldart A particles in gas-fluidized beds, Journal of Particuology, Volume 6, Issue 6, pp 540-548

Yin X., Sundaresan S., 2009, Drag Law for Bidisperse Gas-Solid Suspensions Containing equally sized spheres, Journal of Industrial and Engineering Chemistry Research, Volume 48, Issue 1, pp 227-241

Yang N., Wang W., Ge W., Li J., 2003, CFD Simulation of Concurrent-up Gas-Solid Flow in Circulating Fluidized Beds with Structure-Dependent Drag Coefficient, Journal of Chemical Engineering, Volume 96, Issues 1-3, pp 71-80

Yang N., Wang W., Ge W., Li J., 2004, Simulation of Heterogeneous Structure in a Circulating Fluidized-Bed Riser by Combining the Two-Fluid Model with the EMMS Approach

Zhang D.Z., Vanderheyden W.B., 2002, The Effects of Mesoscopic Structures on the Macroscopic Momentum Equations for Two-Phase Flows. International Journal of Multiphase Flow, Volume 28, Issue 5, pp 805-822

Zou L. M., Guo Y. C., Chan C. K., 2008, Cluster-based drag coefficient model for simulating gas-solid flow in a fast-fluidized bed, Journal of Chemical Engineering Science, Volume 63, Issue 4, pp 1052-1061 
CHAPTER 5: SIMULATION OF FAST FLUIDIZATION FLOW INSIDE THE FIU_CFB FACILITY USING ENHANCED CLUSTER-AWARE DRAG MODELS IN MFIX-TFM

\subsection{Abstract}

An experiment was designed and conducted in order to extract information about fluidization of FCC material inside circulation fluidized bed facility at the Florida International University (FIU-CFB). Static pressure was measured along the fluidization column at different air and solid mass flow rates. In addition, particle clusters of different shapes and sizes were detected in the images obtained from the flow. Measurement data from this experiment was used to set up and validate Two-Fluid Modeling (TFM) simulations of the air-FCC fast fluidization flow using a standard drag model and two cluster-aware drag models for computation of momentum exchange coefficients between gas and solid. For the flow under consideration, simulation results of all models were in decent agreement with the pressure data from the experiment. However, better agreements in terms of pressure values were obtained by the cluster-aware models which complied with lower values of drag forces between the gas and solid.

\subsection{Introduction}

Fluidization is an important process that is used widely today in conversion and utilization of fossil fuels, such as gasification, catalytic cracking, and combustion in circulating fluidized bed risers, and transport reactors. Therefore, it is important to enhance our understanding of the processes involved in fluidization in order to optimize the design of fluidized beds, increase their efficiency, and improve the accuracy of CFD 
models, which can save significant time, budget and burden associated with the experiments. Today, utilization of fine powders in gas-solid systems are of special importance and attention towards the Geldart A group of particles is rapidly growing due to their vastest application in the industrial fluidized beds (Cocco et al., 2014). Geldart A group is a classification of powders known by mean particle diameter of less than $125 \mu \mathrm{m}$ and low particle density of less than $1400 \mathrm{~kg} / \mathrm{m} 3$ (Cocco et al., 2014). As an example, Fluid Cracking Catalyst (FCC), which belongs to the Geldart A group of particles, is used in production of almost three-quarter of all polyolefin in fluidized-bed processes.

Agglomeration of very small particles commonly named as "fines", which includes the Geldart A group, is a typical behavior of cohesive powders used in fluidized bed columns. The occurrence of particle clusters in fluidized beds has been reported as early as in 1975 by Yerushalmi et al. (1975) for ash agglomeration in a fast fluidized bed gasifiers. Presence of FCC cluster in fast fluidization experiments was reported by Li et al. (1991), Yang and Leu (2009), Cocco et al. (2010), Xu and Zhu (2011), and Cocco et al. (2011). Review of a variety of methods for cluster visualization can be found in the work of Harris et al. (2002).

High speed video imaging of the fluidization flow was adopted by Harris et al (2002), Rhodes et al. (1992), Lim et al. (1995), and Gidaspow et al (1989), as a non-intrusive method of cluster visualization near the wall of the riser. Rhodes et al. (1992) observed that swarms of particles were generated from agglomeration of dense flow and were stable as long as pulses of gas were balance by wall frictions and the gravitational forces. 
Harris et al. (2002) obtained blur images of the particle strands at the wall of the experimental unit. These researchers generated useful correlations for particle clusters traveling near the walls of fluidized bed by curve fitting through a large collection of data from the literature. Some of the data that these researchers used, e.g., cluster mean velocity, were obtained from high speed video imaging of the flow, by Gidaspow et al. (1989) and Lim et al. (1995). This indicates the importance of high speed video imaging method, despite drawback such as impairments of image quality due to statics blocking the camera view or reflections from the transparent riser walls.

Quantitative approaches have been developed in the literature to identify particle clusters in both experiment and simulation. Soong et al. (1993) proposed to identify cluster formation as a significant increase of instantaneous solid volume fraction above the local time-averaged values. The threshold for this recognition was two times the standard deviation. Sharma et al. (2000) added definitions like starting and ending life time of clusters. Brereton and Grace (1993) proposed an intermittency index based on voidage fluctuations at a point, which varies from zero for homogenous flow to 1 for swarm of particles.

These criteria were suitable for implementation in the Two-Fluid Modeling approach (developed by van Deemter and van der Laan, 1961) since no information regarding the distances between particles was required. Gomez et al. (2008) developed a criterion related to the time average value of the volumetric solid fraction in a two-fluid modeling of a circulating fluidized bed and reported good qualitative comparison between their 
simulation results and experimental data. However, in continuous representation of the solid phase in TFM, full reliance on solid volume fraction for particle clustering may be somewhat misleading. To better explain this, a computational cell in dilute region of the domain is considered. This cell may not represent clustering because of low content of solid, although there may exist a cluster of 100 particles which occupies exactly the same fraction of the computational cell. For this reason, strength of cohesive interparticle forces is needed to be included as another criterion for clustering formation.

These forces play a major role in fluidization of strongly cohesive particles in Geldart A and C groups by creating heterogeneous structures, called clusters. According to Li et al. (2013), clusters affect the flow significantly by changing the mass and momentum transfers between the gas and solid phases. Many researchers, such as Agrawal et al. (2001), Zhang and Vanderheyden (2002), McKeen and Pugsley (2003), Yang et al. (2003, 2004), Ye et al. (2005a,b, 2008), Qi et al. (2007), Wang et al. (2007 and 2009), Wang et al. (2008), Lu et al. (2009), Igci et al. (2012), and Li et al. (2013), believe that clusters are responsible for significant reduction of the interfacial drag forces between the gas and solid phases. Therefore, dependency of the drag forces on the nature of the attractive interparticle forces plays as important a role as the dependency on two other parameters, i.e., the Reynolds number of the flow around particles and the volume fraction of the solid phase in each computational cell.

Presently, MFIX is a widely known, reliable, and professionally established package for simulation of heat and mass transfer. MFIX accommodates a variety of drag 
models that can be used in TFM simulation of gas-solid particulate flows. One notable drawback of the TFM in MFIX is the absence of cohesive inter-particular forces, such as electrostatic and van der Waals forces between particles. Yet, the direct or indirect addition of models for particle-to-particle attractive and repulsive forces to the transport equations solved in TFM, or to the available drag laws, is missing. There have been several attempts indeed to improve the performance of the MFIX-TFM code in regards to accommodating the influences of heterogeneous structures, such as clusters. Great contributions, such as introducing more complex drag laws, can consider the effect of subgrid-scale heterogeneous structures in TFM simulations. For example, incorporation of the filtered models of Igci et al. (2011) and Milioli et al. (2013) is very appreciable. However, the constitutive models used in these filtered models were obtained from TFM simulations in the absence of the cohesive interparticle forces. This gap can be filled by inclusion of cohesive interparticle forces in the MFIX-TFM code, similar to the inclusion of van der Waals in the MFIX-DEM code (MFIX-2013 Release Notes). In addition, no study has been found in the literature that has implemented the inclusion of the van der Waals forces in the drag laws within the MFIX-TFM code.

In this study, an experiment designed to measure the pressure profile along the riser of a circulation fluidized bed facility is presented. Procedures involved in measuring the boundary conditions, density and size distribution in FCC powder, and calibration of components of data acquisition system are explained. In addition, the presence of particle clusters of FCC inside the column of the test facility will be confirmed. Next, numerical study of the fluidization flow used in the experiment will 
be presented. Benchmark validation of the hybrid models introduced in chapters 2 and 4 of the present dissertation will be presented and common points with the benchmark studies in chapter 2 and 3 will be highlighted.

\subsection{Experimental Study}

\subsubsection{FIU-CFB Test Facility}

A 12 foot circulation fluidized bed was enhanced in design and safety aspects and used for Fluidization experiments for the validation purposes in this dissertation. Figure 5-1 shows the CFB facility which is composed of a Roots blower, distributor plate, acrylic vertical column, $45^{\circ}$ down comer pipe, manually controlled sliding solid feed valve, separation cyclone, inventory, pipe lines, and junctions. In this facility, delivery of air from the Roots blower to the bottom of the fluidization column is through a perforated plate-type distributor placed at the bottom of the transparent riser. The Roots Blower provides up to 10psi pressure difference and can provide up to $200 \mathrm{cfm}$ of air flow in maximum speed of 1800 rpm which supports the air flow rate in the range of 44 to 177 cfm for fast fluidization experiments in the 6 inch diameter-fluidization column. The speed of the motor that runs the blower was controlled through the Variable Frequency Drive (Baldor MN715 VFD) unit with ability to change the speed in 5 rpm resolution. The media grade and permeability of the porous plate of the 316LSS Rolled Sheet category are 40 and 2.9 according to the Mott Corporation data ${ }^{1}$.

\footnotetext{
${ }^{1}$ http://www.mottcorp.com/resource/pdf/LiquidAir_Efficiency.pdf http://www.mottcorp.com/resource/pdf/RS_Perm_all.pdf
} 


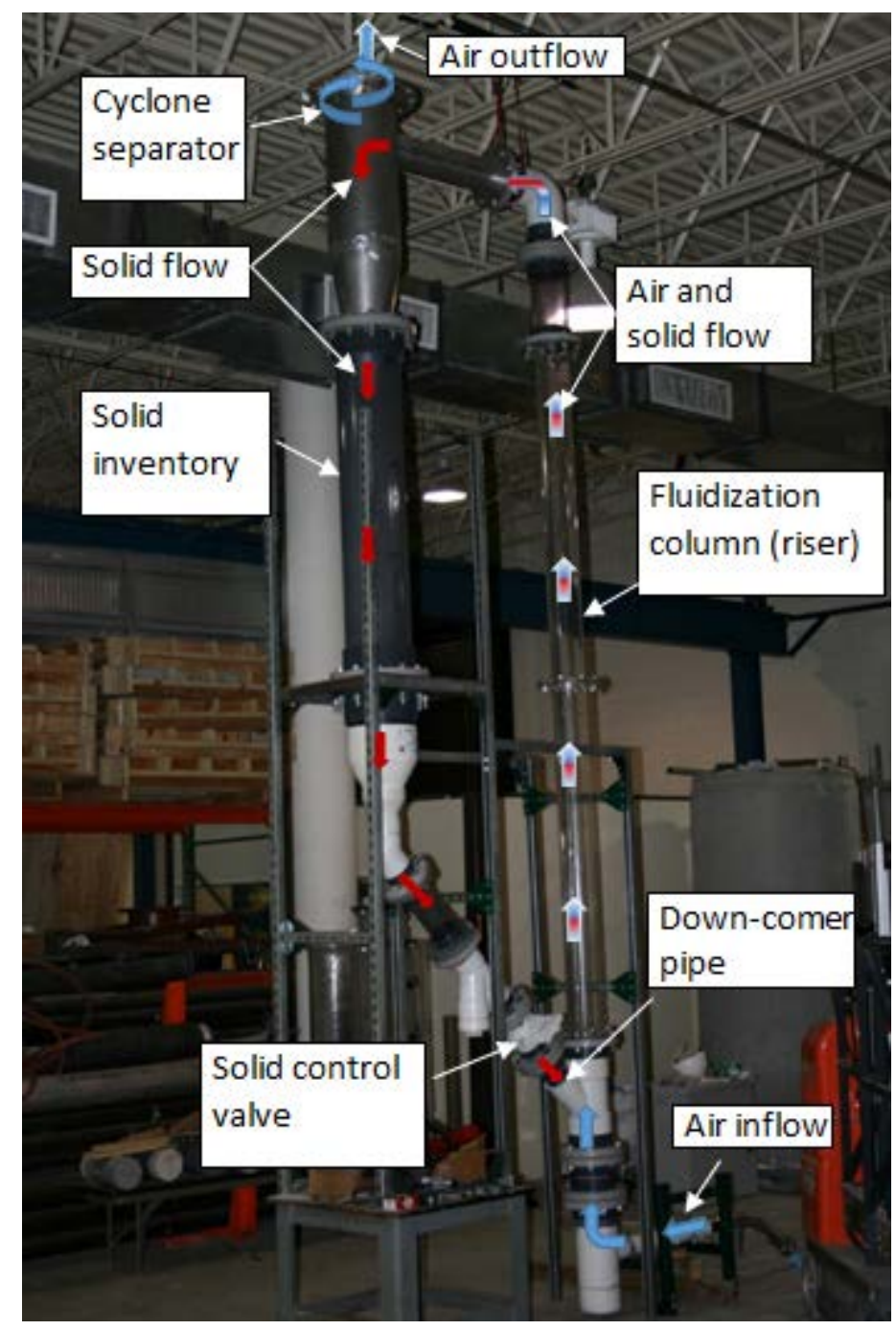

Figure 5-1 Circulation fluidization bed (CFB) for fluidization test at FIU

\subsubsection{Air Flow Rate Measurement}

Air volumetric flow rate was measured using a Omega ${ }^{\circledR}$ Fl45230A flow meter with the accuracy of \pm 2 percent of the measured flow rate. Recorded data from the flow meter required correction since factory scales were based on standard test conditions. Eq. (5.1) shows the corrections to the flow rate from standard conditions done by the manufacturer to actual values in the real tests. In this expression, ACFM, SCFM, R, and T refer to the 
actual and standard pressures, relative humidity, and temperature. Respectively. The std, act, and sat refer to the standard, actual, and saturation in this expression.

$$
A C F M=S C F M \times \frac{P_{\text {std }}}{P_{\text {act }}-P_{\text {sat }} \times R} \times \frac{T_{\text {act }}}{T_{\text {std }}}
$$

Pressure and temperature data were obtained from a gauge-type PX309 transducers from Omega Engineering® pressure transducer with accuracy of $\pm 0.5 \%$ of the operational range and a T-Type thermocouple with accuracy of $\pm 1 \%$ of the measurement, respectively. Relative humidity $(\mathrm{RH})$ of the air in the system was measured at an opened port just before the distributor port using an EXTECH RH ${ }^{\odot}$ meter with accuracy of $\pm 2 \%$ of the reading. The RH value varied between 45 to 50 percent inside the system and the effect of this variation was less than 0.2 percent in Eq. (5.1). Saturation pressure of the air was obtained from the air psychometrics charts at the measured temperature. Details of calibration of the FieldPoint module and pressure transducer will be presented later on this chapter. In addition, density in the test condition was calculated according to the ideal gas law and through a Real-time LabView ${ }^{\circledR}$ VI version 8.5 program, which processed the data in form of current from an AI-110 FieldPoint module. This quantity was necessary for calculation of the mass flow rate of the incoming air to the system. Figure 5-2 shows the LabView ${ }^{\circledR}$ VI program designed for current-to-pressure conversion and calculation of the density. 


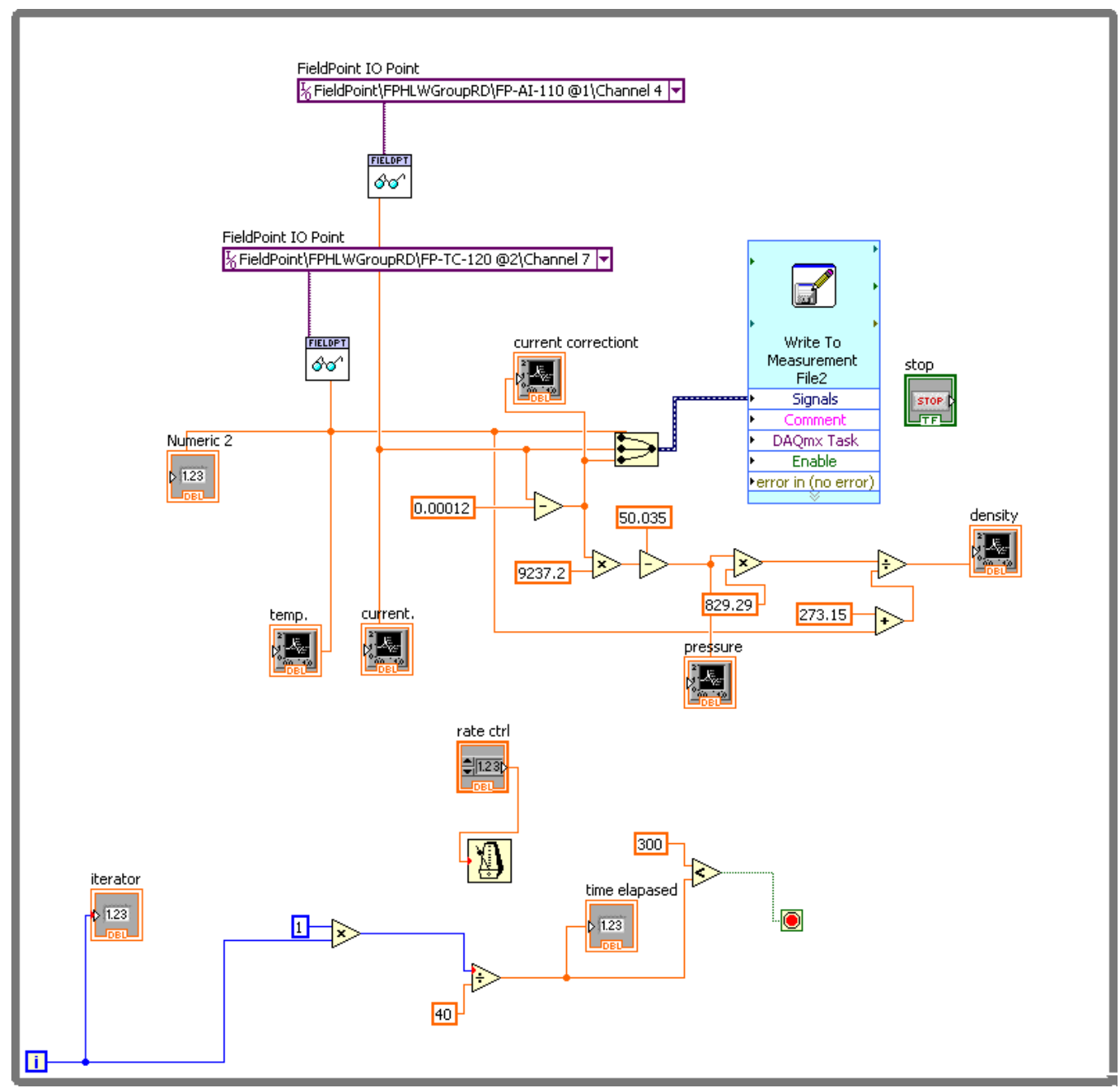

Figure 5-2 LabView $^{\circledR}$ VI designed for communication with pressure transducer and thermocouple on the Field point2010 for the purpose of data reception and storage.

\subsubsection{Solid Density and Flow Rate Measurement}

Density of the FCC powder was measured directly in a weight to volume method experiment. Before the test, powder was mixed for 1 minute all over the barrel. The powder was sampled from different depth locations to better represent the material. 5 samples were taken from the barrel and each sample was weighed 4 times using a scale with less than a \pm 0.1 gr error and measured volumetrically with about $\pm 2 \mathrm{ml}$ visual error. 
In this approach, density of the powder was obtain as $845 \mathrm{~g} / \mathrm{cc}$ within $\pm 17 \mathrm{gr} / \mathrm{cc}$ of measurement error.

Solid input was provided by the weight of the solid in the inventory component of the system and was controlled by a manually-controlled sliding valve design. During the experiment, a steady condition was maintained, where by visual inspection, the amount of solid in the inventory was checked to be at a certain level. This condition was guaranteed by negligible solid loss rate from the system at the cyclone separator. To reach this at any speed of the blower, valve position was strictly controlled and adjusted to keep the solid level in the inventory close to constant.

A solid mass flow rate experiment was designed in order to measure the solid circulation flow rate in the system. Solid flow rate measurement is needed to create accurate boundary conditions in the MFIX simulations to replicate the CFB experiments for validation purposes. Another purpose of this test was to ensure that gravitational force of the material above a certain level in the inventory component was sufficient to provide a constant solid influx to the system for a constant valve opening by a good approximation. This could be only proven under the condition that data from this experiment did not exhibit constant decrease of solid mass flow rate for each valve position when the inventory and down comer pipes would lose material. This condition was met in an experiment designed to repeat measurements for a constant valve opening when the system was not running. Validity of the results of this experiment was extended to normal system operation based on the fact that force of pressures difference between points 
above the inventory (close to atmospheric pressure) and behind the gate of the solid valve (back pressure) was negligible in comparison to the accumulative weight of the material in the down comer pipe and the inventory. It was observed that even during the system operation, steady flow of solid was not disturbed by the back pressure from the incoming air.

Figure 5-3 shows the components of the experiment designed for solid mass flow rate. In this experiment, the distributor plate was indicated as the reference line and solid valve gate was kept open until solid level reached the horizontal red line displayed in the left picture in Figure 5-3. The position of the valve was varied in 0.25inch intervals. Calculation of the characteristic volume $\left(\mathrm{V}_{\mathrm{c}}=0.0067 \mathrm{~m}^{3}\right)$ was performed in the SolidWorks and solid mass flow rate was calculated from Eq.(5.2). In this equation, density and normal packing ration are $\rho=845 \mathrm{~g} / \mathrm{cc}$ and $\varepsilon_{\max }=0.63$ for spent FCC and obtained from measurement and manufacturer, respectively.

$$
\dot{\mathrm{m}}=\frac{\varepsilon_{\mathrm{max}} * \mathrm{~V}_{\mathrm{c}}{ }^{*} \rho_{\mathrm{FCC}}}{\text { Time }}
$$
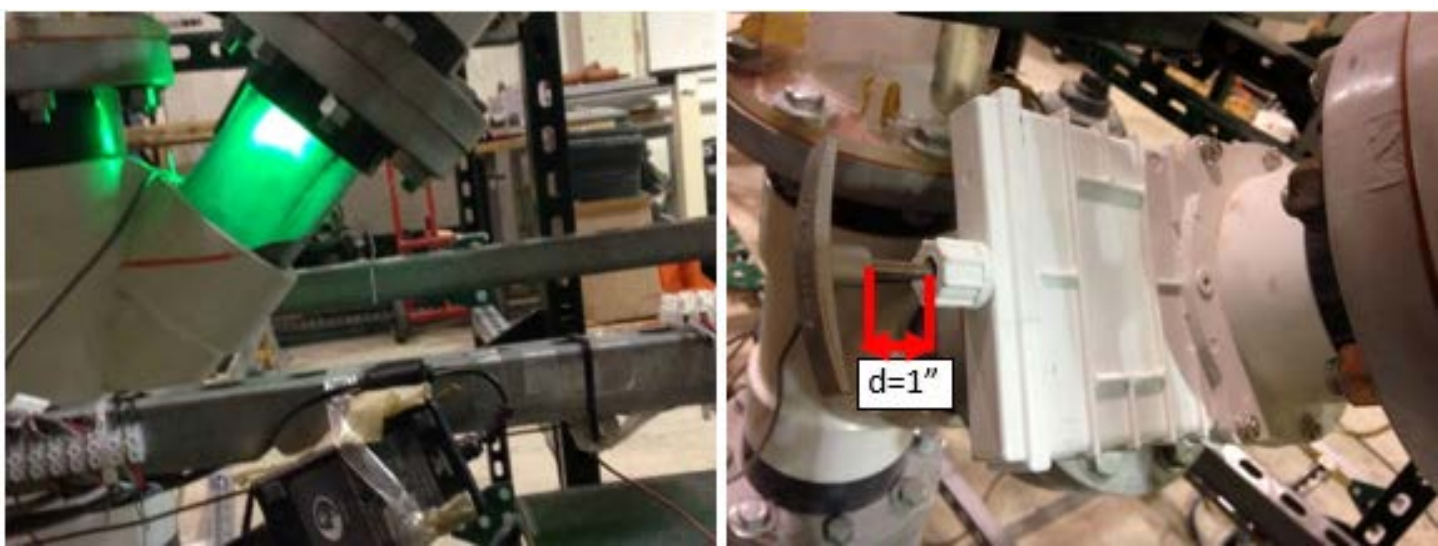

Figure 5-3 Solid mass flow rate measurement experiment (on left), and` solid sliding valve at the fully closed position $\mathrm{d}=1$ ”). 
Figure 5-4 shows the results of the solid mass flow rate experiment. The red bars shows the $95 \%$ confidence intervals which show small deviations for average values. In addition, for each value position, measurements did not exhibit constantly decreasing values and instead a trend of fluctuation of around mean values was observed. This proved that existing mass in the inventory part for each measurement was enough to ensure a constant solid mass flow rate.

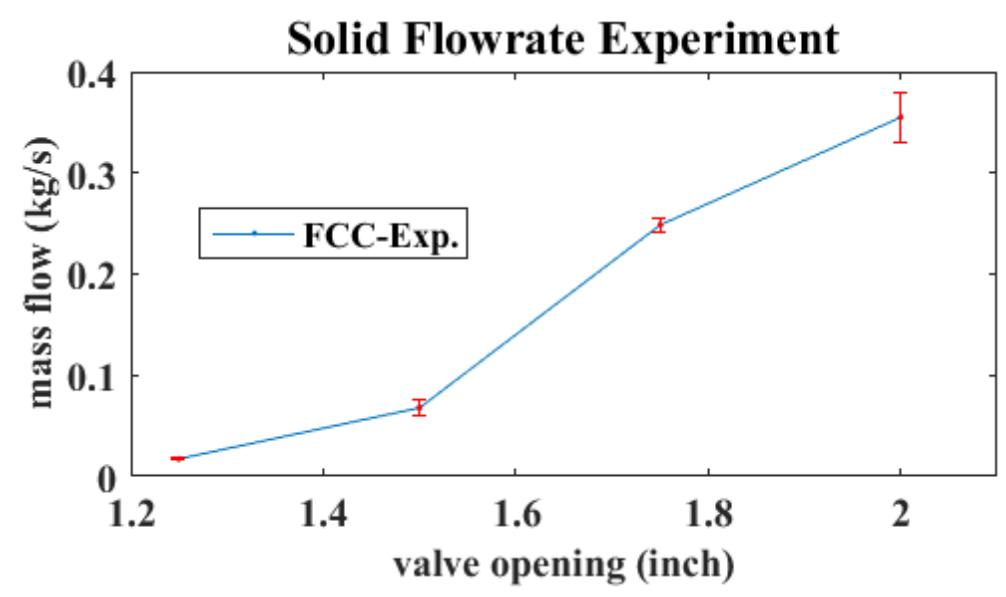

Figure 5-4 Mass flow rate measurement for FCC in different valve openings

Table 5-1 shows the solid mass flow rate values for certain valve positions obtained by interpolation of the data presented above. The last column in Table 5-1 shows the values of the solid volume fraction $\left(\varepsilon_{s}\right)$ calculated at the solid inlet port. This port is the junction of the 45 degree down comer and the vertical column, where solid enters the column with a certain velocity. Calculation is based on the ration of the exposed area created by valve opening to the cross sectional area of the down comer pipe by considering a loosely packing ration of 0.45 for flowing FCC. Construction of the exposed area and related calculations were performed in the SolidWorks ${ }^{\circledR}$ application. 
Table 5-1 Mass flow rate and Mass flux calculated for the FCC in different valve openings

\begin{tabular}{|c|c|c|c|}
\hline $\begin{array}{c}\text { Solid valve } \\
\text { position (inch) }\end{array}$ & $\begin{array}{c}\text { Solid valve } \\
\text { opening (units) }\end{array}$ & $\begin{array}{c}\dot{\mathrm{m}}_{\mathrm{FCC}} \\
\text { (kg/s) }\end{array}$ & $\begin{array}{c}\text { Solid volume } \\
\text { fraction }\end{array}$ \\
\hline 1 & 0 & 0.0000 & 0.00 \\
\hline 1.125 & 18 & 0.0083 & 0.012 \\
\hline 1.188 & 19 & 0.0124 & 0.018 \\
\hline 1.25 & 20 & 0.0166 & 0.024 \\
\hline 1.313 & 21 & 0.0283 & 0.031 \\
\hline 1.375 & 22 & 0.3999 & 0.036 \\
\hline
\end{tabular}

\subsubsection{Performance Checkup Test of Data Acquisition Unit}

Performance tests and calibrations of the FieldPoint AI-100, AI-110 units was done using a current evaluation experiment. In this procedure, a current source generated by an Omega Engineering® ${ }^{\circledR}$ power source and was measured by Agilent U12252 multimeter with resolution and accuracy of $1 \mu \mathrm{A}$ and \pm 0.6 percent of the reading, respectively. The schematic and original view of the test is displayed in

Figure 5-7. In this test the accuracy of the FieldPoint modules was less than 0.1 percent of the reading.
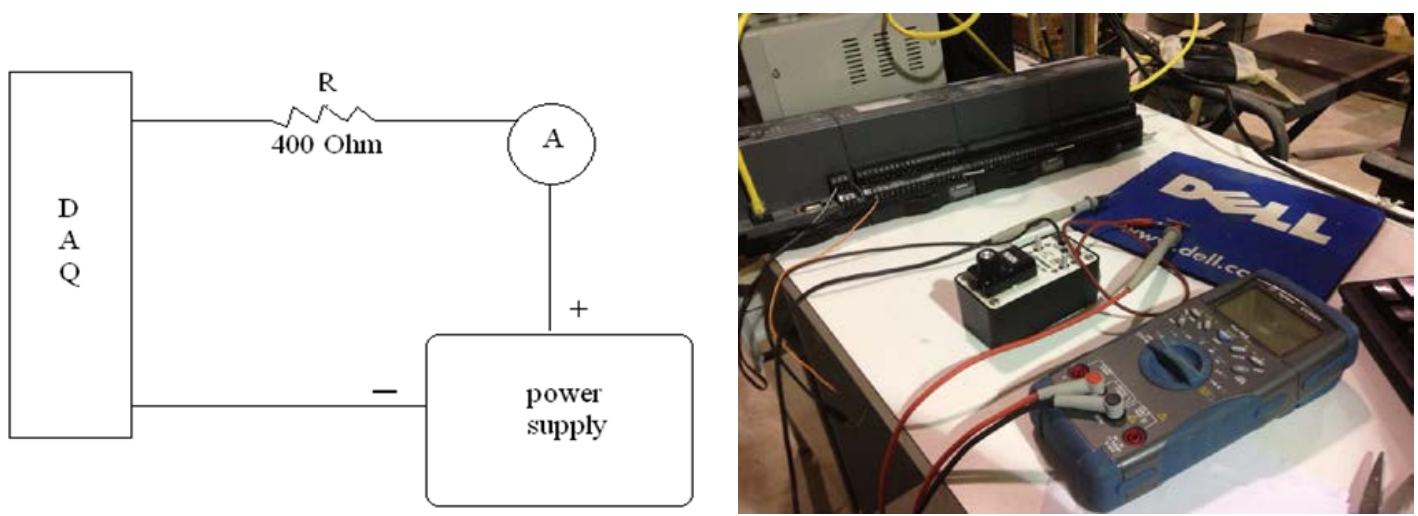

Figure 5-5 Schematic of the first (on left) and second (on right) data performance checkup test for the data acquisition module with Filed point 2010 control module 


\subsubsection{Performance Checkup Test of Pressure Transducers}

Pressure data in this experiment were obtained using 0-2 psig and 0-5 psig PX209 and PX309 type transducers from Omega Engineering ${ }^{\circledR}$. Transducers produced a 4 to $20 \mathrm{~mA}$ current-type response with a response time of less than $1 \mathrm{~ms}$ and accuracy of $\pm 0.25 \%$ and $\pm 0.5 \%$ of the operational range, respectively. National Instruments FieldPoint ${ }^{\odot}$ units AI100 and AI-110 modules collected the excitation response from the transducers and produced current output with update rate, resolution and gain error of $2.8 \mathrm{~ms}, \pm 15 \mu \mathrm{A}$, and $0.09 \%$, respectively. LabView ${ }^{\circledR} 8.5$ VI was used to program and control the connection between the transducers and the channels of the FieldPoint units.

Calibration of pressure transducers was an extremely important step in this experiment. Initial measurement of pressure along the riser using 0-2 psig PX209 type transducers from Omega Engineering ${ }^{\circledR}$ showed pressure values in the range of 0 to 0.2 psi for speeds of the blower up to $630 \mathrm{rpm}$. This sensitivity required careful calibration of the transducers to avoid errors as large as the measure pressure. Figure 5-6 shows a simple of performance checkup test and calibration of the pressure transducers and this test was performed for 7 transducers. In this test, a monometer and two pressure gauges in ranges of 0-5 and 0-10 psi were used to measure and control the excitation pressure from a regulated pressure source. Reading pressure from a manometer with maximum uncertainty of \pm 0.001 psi made it possible to control the excitation pressure with increments of 0.02psi in the test. The results are shown in Figure 5-6(a) and Figure 5-6(b) (square symbols) which follow the trend of factory data and deviation from the factory data was less than 1.5 percent. The error bars associated with 95\% confidence interval 
were generated (Figure 5-6-a) to ensure that appropriate pressure increments were used for curve fitting to the measurement data. It was realized that confidence intervals at 0.02psi increments did not overlap and the generated fit was used for the calibration purpose.

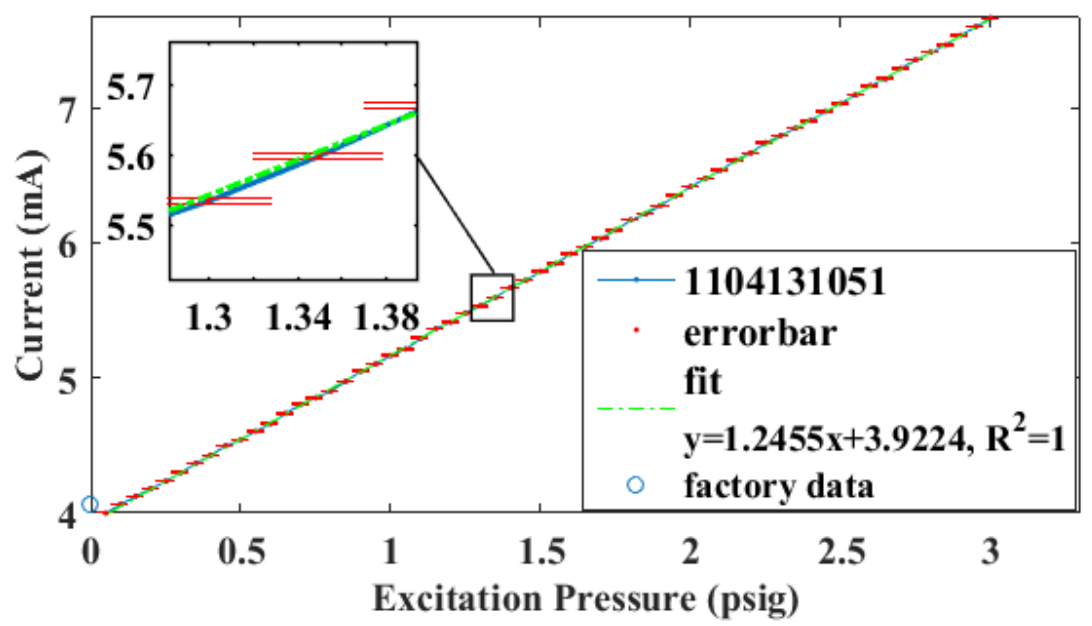

(a)

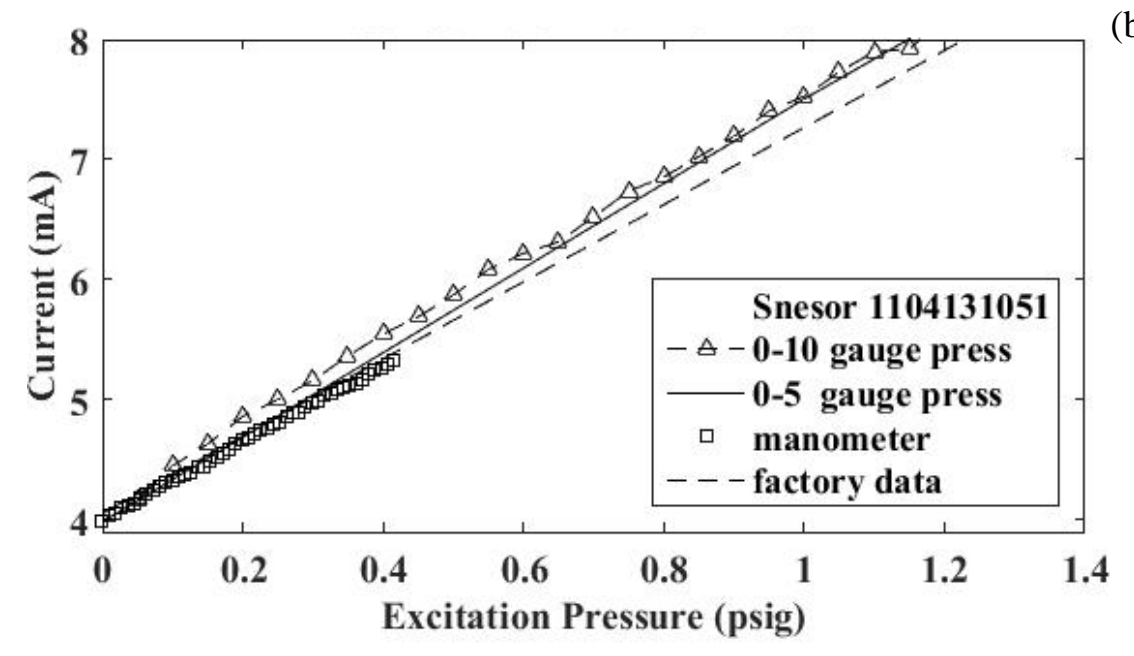

(b)

Figure 5-6 Sample performance check of pressure transducers (a) using increment of 0.01psi for excitation pressures controlled by manometer (b) comparison of different methods and factory data 


\subsubsection{Particle Size Distribution Analysis}

In order to determine the size distribution of the available sample, a series of 12 sieving tests was performed at FIU. In this procedure for each test, samples of powder were taken from different depth and radial locations in the drum poured on a stack of six sieves placed on top of each other. Sieves sat on each other in the order of the coarsest sieve with pores as large as $600 \mu \mathrm{m}$ on the top to the finest sieve with pores as small as $32 \mu \mathrm{m}$ in the bottom and the stack was shaken for 20 minutes in each test. Weight of each sieve was measured before and after the test using a precision weighing scale with accuracy of \pm 0.1 gram, in order to obtain the net weight of particles entrapped in each sieve. Eq. (5.3) shows the expression used to find the mean diameter of the particles in this test. N refers to number of size categories (sieves), $\mathrm{x}$ refers to the ratio of the material weight in

the sieve to the total weight of the sample, i.e., $w_{i} / \sum_{i=1}^{N}\left(w_{i}\right)$, and $d_{i}$ is the average diameter of the size categories. The last parameter is obtained by averaging the diameter of the pores of the $\mathrm{i}^{\text {th }}$ sieve and its lower sieves. This quantity was $16 \mu \mathrm{m}$ for the finest sieve in this test which had the pores of $32 \mu \mathrm{m}$, since the collector was located under this sieve.

$$
\mathrm{d}_{\text {mean }}=1 / \sum_{\mathrm{i}=1}^{\mathrm{N}}\left(\mathrm{x}_{\mathrm{i}} / \mathrm{d}_{\mathrm{i}}\right)
$$

Figure 5-7 displays the apparatus of the test including the sieves, shake machine, support frames and the controller unit. On the right side in this figure, results of 3 sieving tests is shown. Each test was performed using different combination of sieves of different pore sizes and was repeated for 4 times. The overall average of this test was a mean diameter of $79.03 \mu \mathrm{m}$. 

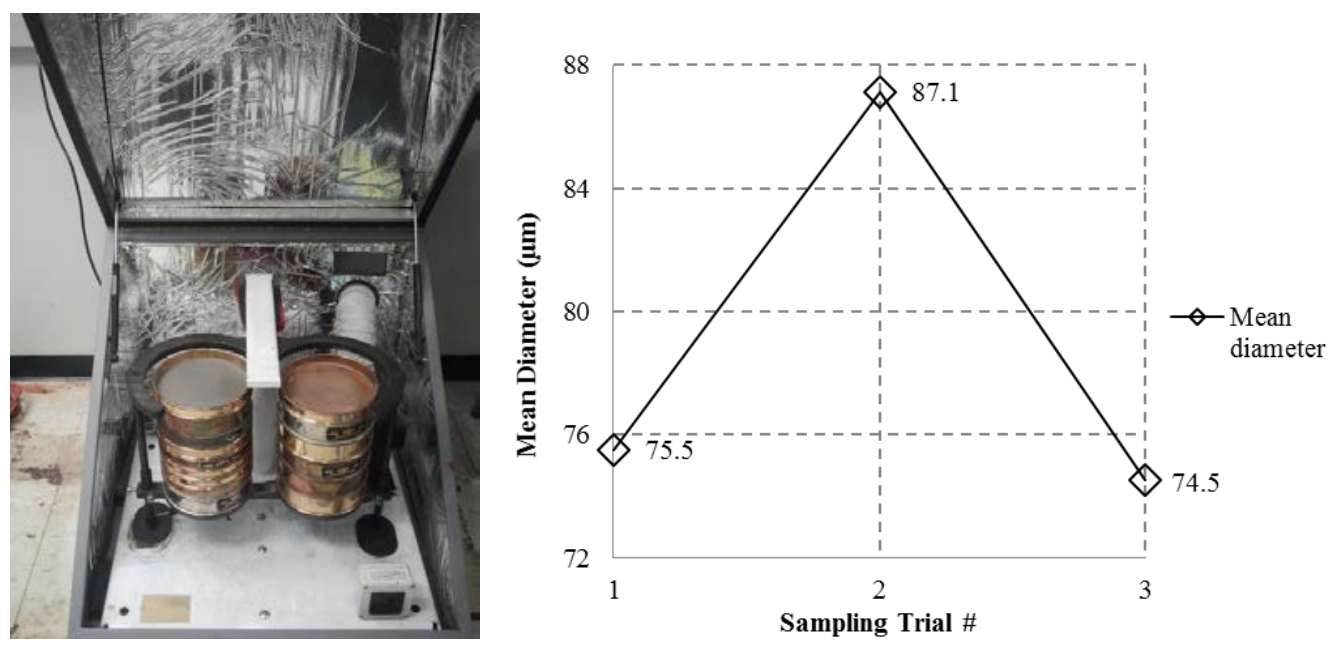

Figure 5-7 Shaker machine, implementing sieving mechanism to categorize particles based on size

\subsubsection{Pressure Measurement in the Fast Fluidization Experiment}

The CFB was operated under different gas volumetric and solid mass flow rates. In this experiment, the air flow rate was adjusted by the speed of the blower (540 and $570 \mathrm{rpm}$ ) and the solid flow rate was adjusted by the sliding valve position. The air flow rate was measured using the flowmeter and correction to the measured flow rates were applied based on the Eq. (5.1). For this purpose the air pressure, temperature, and flow rate in the system were continuously measured and averaged during the period of data collection. The correction factors were calculated, as shown in Table 5-2. The solid mass flow rates at different valve openings were according to Table 5-1. Here, calculation of solid flux was based the wetted area of the solid in the inlet port, which is $\mathrm{A}_{\text {wett }}=\varepsilon_{\mathrm{FCC}} * \mathrm{~A}_{\text {inlet }}$, where $\boldsymbol{\varepsilon}_{\mathrm{FCC}}$ was according to Table 5-1. 
Table 5-2 Test conditions for different air flows and FCC mass fluxes

\begin{tabular}{|c|c|c|c|c|c|c|c|c|c|}
\hline Test ID & $\begin{array}{c}\mathrm{T} \\
\left({ }^{\circ} \mathrm{C}\right) \\
\pm 1 \% \\
\end{array}$ & $\begin{array}{c}\mathrm{P}_{\mathrm{m}}{ }^{[\mathrm{a}]} \\
\text { (pascal) } \\
\pm 15\end{array}$ & $\begin{array}{c}\dot{\mathrm{V}}_{\mathrm{STD}}{ }^{[\mathrm{b}]} \\
\left(\mathrm{m}^{3} / \mathrm{hr}\right) \\
\pm 2 \% \\
\end{array}$ & $\begin{array}{c}\dot{\mathrm{V}}_{\mathrm{act}}{ }^{[\mathrm{c}]} \\
\left(\mathrm{m}^{3} / \mathrm{hr}\right) \\
\pm 2 \% \\
\end{array}$ & $\begin{array}{c}\rho \\
\left(\mathrm{kg} / \mathrm{m}^{3}\right) \\
\pm 0.2 \% \\
\end{array}$ & $\begin{array}{l}V_{\text {air }} \\
(\mathrm{m} / \mathrm{s}) \\
\pm 2 \% \\
\end{array}$ & $\begin{array}{c}\dot{\mathrm{m}}_{\mathrm{FCC}} \\
(\mathrm{kg} / \mathrm{s}) \\
\pm 8 \% \\
\end{array}$ & $\begin{array}{c}\ddot{\mathrm{m}}_{\mathrm{FCC}} \\
\left(\mathrm{kg} / \mathrm{m}^{2} . \mathrm{s}\right) \\
\pm 10 \% \\
\end{array}$ & $\begin{array}{c}\mathrm{Pp}^{\mathrm{d}} \\
\text { (pascal) } \\
\pm 7\end{array}$ \\
\hline 540-18 & 27.8 & 3861.1 & 80.5 & 80.5 & 127 & 1.23 & 0.0083 & 9.475 & 2986.1 \\
\hline $540-20$ & 26.9 & 3981.3 & 80.0 & 79.7 & 1. & 1.21 & .0166 & 9.475 & 3085.6 \\
\hline 540 & 30 & 410 & 80.5 & 81.3 & & \pm & 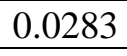 & 12. & 3210.1 \\
\hline $540-22$ & 31.4 & 4184.6 & 80.0 & 81.1 & 1.2( & 1.24 & 399 & 15.217 & 3297.1 \\
\hline $570-18$ & 33.2 & 4081 & 88.0 & 90.0 & & 1.37 & 0.0083 & 9.475 & 3234.9 \\
\hline 570-19 & 36.8 & 413 & 88.0 & 91.4 & & 1.39 & 24 & 9.437 & 3284.7 \\
\hline $570-21$ & 33.9 & 4231.1 & 88.0 & 90.2 & & 1.37 & 0.0283 & 12.506 & 3384.2 \\
\hline $570-22$ & 30.8 & 4361.6 & 87.0 & 87.8 & 1.21 & 1.34 & 0.0399 & 15.217 & 3546.1 \\
\hline
\end{tabular}

${ }^{a}$ Pressure at the meter (gauge) ${ }^{\mathrm{b}}$ Flow at standard condition $\left(\mathrm{T}\right.$ air $=20^{\circ} \mathrm{c}, \mathrm{P}=1 \mathrm{~atm}$ )

${ }^{\mathrm{c}}$ Flow at actual condition $\quad{ }^{\mathrm{d}}$ Pressure below the distributor plate (gauge)

Static pressure along the acrylic riser was collected via transducers placed at specific heights and corrected by means of calibration information obtained for each transducer, such as shown in Figure 5-6-a. For each test, data was collected and averaged after the system reached steady operation, i.e., the solid surface did not change in the inventory. Figure 5-8 shows a sample of data collection during 5 minutes of steady system operation. Data points are sparsely displayed, i.e., representing every 10 point, to avoid cluttered representation. Results show that with the help of precise calibration of transducers, distinguishable pressure values could be collected and recorded. In this representation, pressure ports are labeled successively, where 1 shows the lowest port, i.e., for the port that is $5.7 \mathrm{~cm}$ above the distributor, and 7 shows the port that is close to the outlet of the fluidization column. 
In addition, pressure was measured at a location $10.7 \mathrm{~cm}$ below the distributor (called as plenum pressure, or $\mathrm{Pp}$ ) using a manometer with maximum uncertainty of \pm 0.001 psi. This pressure was obtained in order to complete the information regarding pressure for the validation purpose explained in the next section. This data was obtained for all the cases presented Table 5-2 and was time averaged over 5 min of steady operation of the fast fluidization flow cases.

In order to obtain the pressure profile along the fluidization column, pressure data at each port was time averaged for all the cases studied in this dissertation. Figure 5-9 shows the time averaged profile of pressure for air flows at two speeds of the blower, i.e., 540 and $570 \mathrm{rpm}$. Flow of air in these cases was sufficient to establish a fast/circulating fluidization regime of the flow, where a pattern of rigorous solid back mixing in the fluidization column with circulation of solid in the system was observed. In addition, gas velocity in the experiments falls into the range indicated for fast fluidization flow by Kunii \& Levenspiel (1991). The results show increase of pressure riser by increase of solid mass flow rate into the system. However, variable frequency drive (VFD) controlled the speed of the blower precisely and constant air flows were provided to the system according to Table 5-1. 


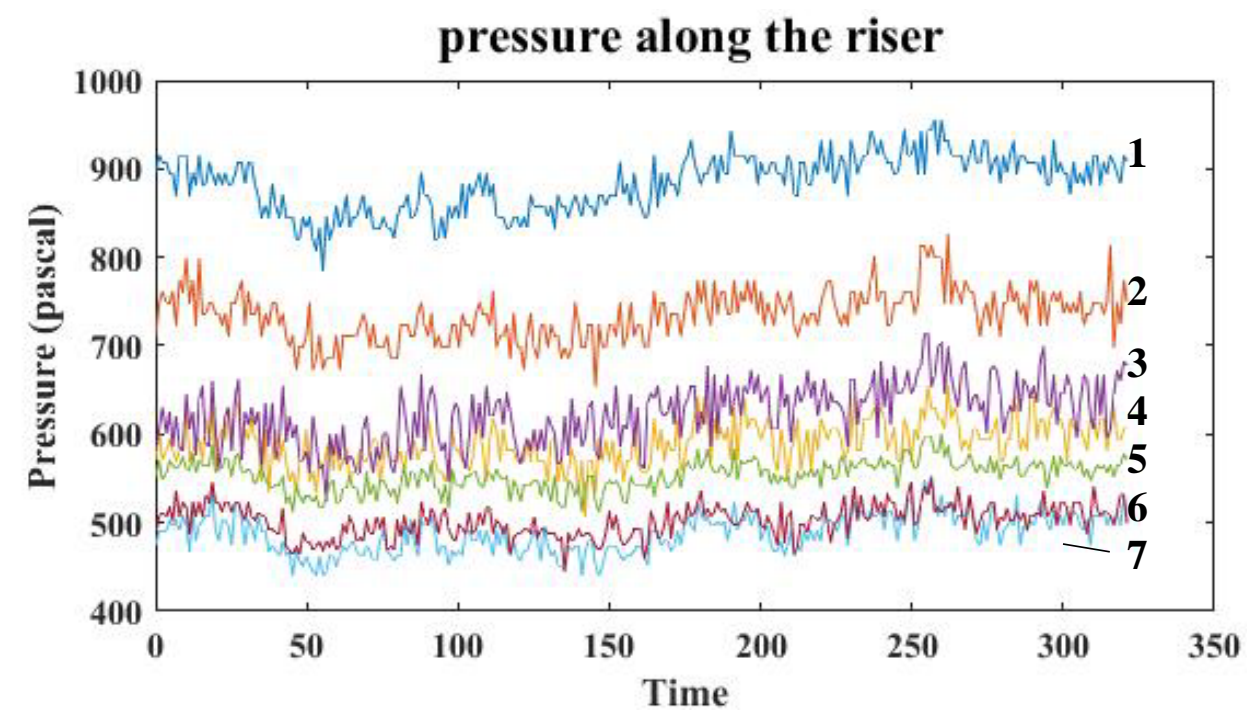

Figure 5-8 Pressure data for the case 540-22, $\mathrm{V}_{\text {air }}=1.24 \mathrm{~m} / \mathrm{s}$ and $\dot{\mathrm{m}}_{\mathrm{FCC}}=40(\mathrm{~g} / \mathrm{s})$

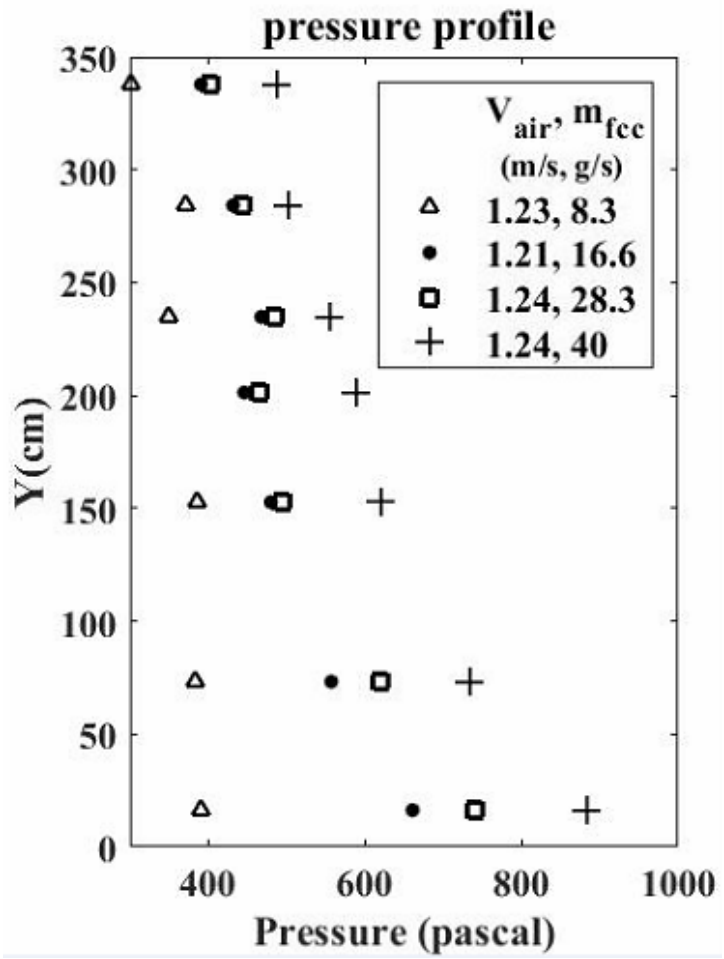

(a)

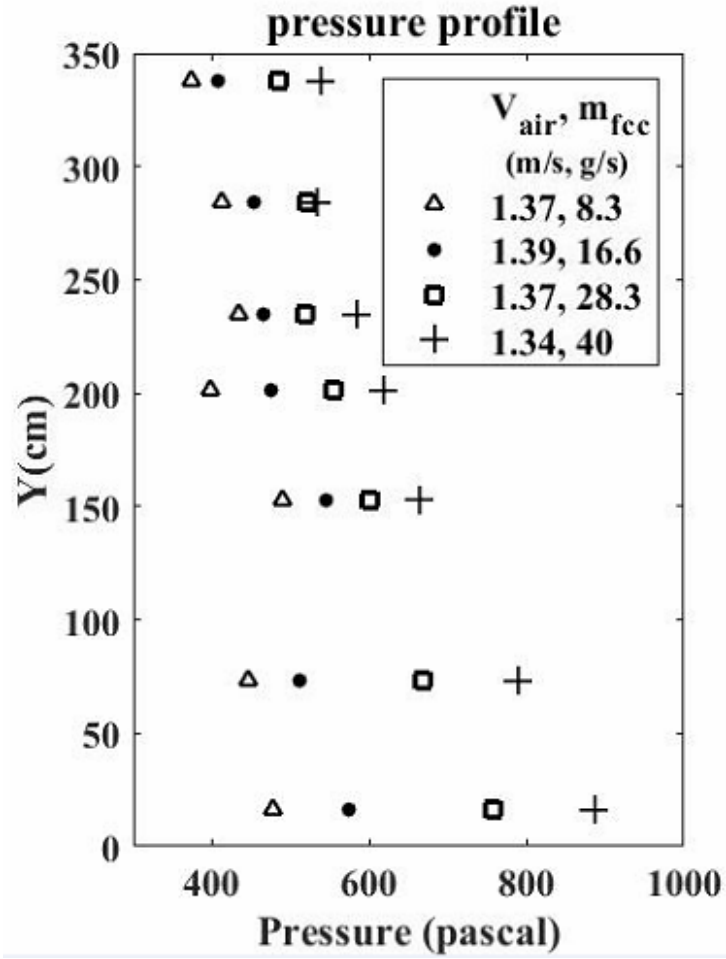

(b)

Figure 5-9 Time-average pressure profile along the fluidization column of FIU-CFB for different air flow and solid mass flow rates (a) blower speed $=540 \mathrm{rpm}$ (b) blower speed $=570 \mathrm{rpm}$ 


\subsubsection{Cluster Detection Inside the Fluidization Column Using High Speed Camera}

Shadow sizing technique was used for detection of clusters from by FCC particles in our experimentations. This technique was used to capture the shadow of the particles, where particles are backlit with a light source and a camera acquires the shadow image of the particles. The light source was placed on the opposite side of the camera allowing the camera to capture the shadow of the solid particle flow in the riser and the camera was connected to the computer, which could control the time and exposure of the camera lenses. A high-speed camera (Vision Research v5.0) that had 3800 pps shooting capability at a resolution of $512 \times 512$ pixels was used for shooting purpose. The maximum frame rate was 60,000 pixels per second (pps) at a resolution of 256 pixels in horizontal resolution and 32 pixels in vertical resolution. A telocentric lens (Edmund Optics Inc. 55-350) was used, that had a horizontal field of view of $88 \mathrm{~mm}$ and a depth of field of $1 \mathrm{~mm}$ according to the manufacturer's technical specification sheet (Edmund Optics, 2011). An LED light source was placed behind the particles to provide an even illumination of the flow field, according to Figure 5-10(a). Clusters were detected in 1000 of images captured by the high speed camera. One difficulty with strongly cohesive particles was separation of statics (particles or clusters sticking to the riser walls) from the floating particles or clusters. Figure 5-10(b) shows an original image taken from the riser section of the FIU-CFB facility including statics. Matlab scripts were used to remove the statics, also referred to as noise, and to detect clusters based contrast thresholding. Few out of many clusters are shown in Figure 5-10(c). 


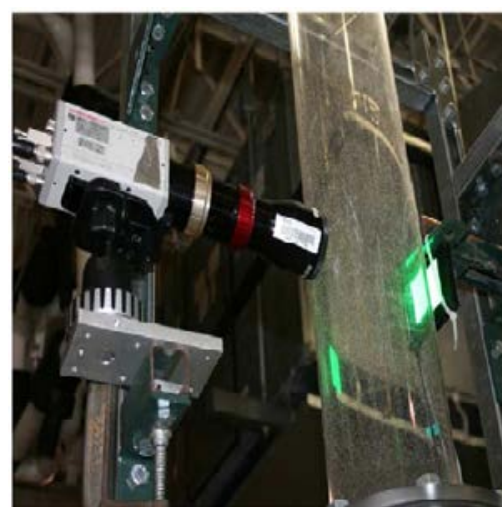

(a)

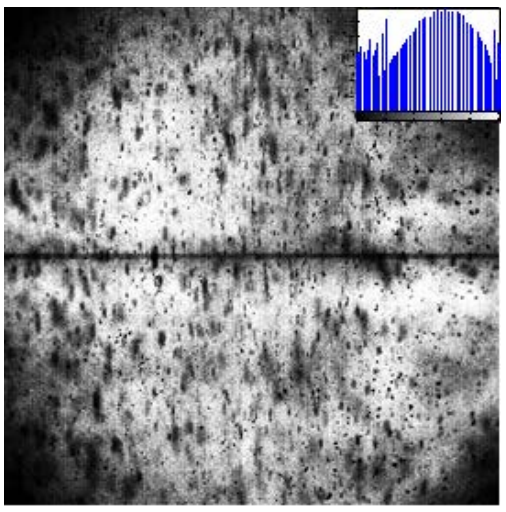

(b)

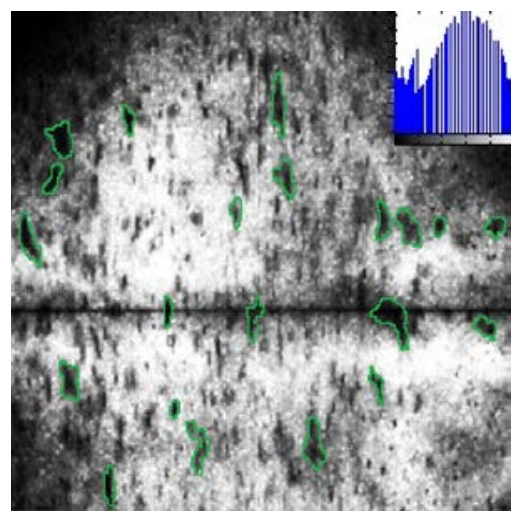

(c)

Figure 5-10 Cluster visualization in fast fluidization experiment (FIU-CFB). (a) Imaging set up, (b) Image with statics on the riser wall (c) noise-free image and portray of few clusters

\subsection{Simulation of Fluidization Flow inside FIU-CFB Facility}

\subsubsection{Simulation Methodology}

An attempt to benchmark validation of the cluster-aware drag model using MFIX-TFM tool is presented in this section. Presence of particle clusters in the flow was proven according to the images of high speed camera. Thus, it was expected to obtain better performance from a cluster-aware drag model than a standard drag model which is based on homogenous particle distribution. The problem consisted of flow simulation of a specific case presented in Table 5-2 and Figure 5-9 and comparison between pressure profile of the simulation and measurement. In this framework, Two-Fluid Modeling simulation of the case designated as 570-22, i.e., $\mathrm{V}_{\text {air }}=1.34(\mathrm{~m} / \mathrm{s})$ and $\dot{\mathrm{m}}_{\mathrm{FCC}}=400(\mathrm{~g} / \mathrm{s})$, is considered. A two dimensional (2d) axisymmetric domain, Figure 5-11, was constructed in the MFIX based on the fact that mass, momentum, and kinetic energy must conserve between the $3 \mathrm{~d}$ and $2 \mathrm{~d}$ cases. Eq. (5.4) to Eq. (5.7) show the procedure to find the inlet port diameters for the $2 \mathrm{~d}$ case which can produce an equivalent three dimensional (3d) 
system. In the case of FIU-CFB, the down comer pipe delivered the solid to the system at a 45-degree angle and area of a $8.5 " \times 6$ " oval was considered. Similar procedure is repeated to find the outlet port diameter for an equivalent $2 \mathrm{~d}$ axisymmetric case.

$$
\begin{gathered}
\dot{\mathrm{m}}_{2 \mathrm{~d}}=\dot{\mathrm{m}}_{3 \mathrm{~d}}=\dot{\mathrm{m}} \Rightarrow \rho_{\mathrm{s}} \mathrm{V}_{\text {in-2d }} \mathrm{A}_{\text {in-2d }}=\rho_{\mathrm{s}} \mathrm{V}_{\text {in-3d }} \mathrm{A}_{\text {in-3d }} \\
\mathrm{m}_{2 \mathrm{~d}} \mathrm{~V}_{\text {in-2d }}=\mathrm{m}_{3 \mathrm{~d}} \mathrm{~V}_{\text {in-3d }} \Rightarrow \mathrm{V}_{\text {in-2d }}=\mathrm{V}_{\text {in-3d }}=\mathrm{V}_{\text {in }} \\
\frac{1}{2} \dot{\mathrm{m}}_{2 \mathrm{~d}}\left(\mathrm{~V}_{\text {in-2d }}\right)^{2}=\frac{1}{2} \dot{\mathrm{m}}_{3 \mathrm{~d}}\left(\mathrm{~V}_{\text {in-3d }}\right)^{2} \Rightarrow \mathrm{V}_{\text {in-2d }}=\mathrm{V}_{\text {in-3d }}=V_{\text {in }} \\
\mathrm{A}_{\text {in-2d }}=\mathrm{A}_{\text {in-3d }} \Rightarrow \mathrm{d}_{2 \mathrm{~d}-\mathrm{in}}=\frac{\mathrm{A}_{\text {in-3d }}}{\pi \mathrm{d}_{\text {riser }}}
\end{gathered}
$$

In the case of B.C. for the solid, more precise information was provided to MFIX by assigning the velocity components at the solid inlet port. For this purpose, the magnitude of the velocity was obtained from the mass flow rate of solid, i.e., $V=\dot{m} /\left(\rho_{s} A_{\text {inlet }} \varepsilon_{s}\right)$, and components of solid velocity, i.e., $\mathrm{V}_{\mathrm{x}}=-\mathrm{V}^{*} \cos (\Theta)$ and $\mathrm{V}_{\mathrm{y}}=-\mathrm{V}^{*} \sin (\Theta)$, where computed by plugging $\Theta=45^{\circ}$, which is the angle of down comer. Here, the solid volume fraction at the inlet port, i.e., $\varepsilon_{\mathrm{s}}$ is according to the Table 5-1. Negative signs indicate the inflow of solid to the domain was in opposite direction with regards to the axis convention of the coordinate system. After that, the mass flow rate of the solid was checked using MFIX B.C. checking toolbox to make sure correct mass flow rate would be attained at the solid inlet port.

In the case of inlet B.C. for the upward incoming airflow from the blower (B.C.air), the surface of the distributor plate (called as P0) was considered as the air velocity inlet 
boundary. This boundary had a known superficial velocity (volumetric flow rate divide by the pipe cross sectional area) and zero solid volume fraction, which was a realistic condition since the role of this porous plate was provision of pure air. The reason that another location such as the location of the first transducer, also called as P1, was not a proper air inlet boundary due to lack of information about solid volume fraction and gas velocity.

The pressure-type outlet boundary was considered for the exit section of the computational domain in the simulations. The values of pressure at this B.C. were set to the pressure obtained from the most top pressure transducer in the experiment. In fact, setting the pressure to atmospheric values was not a realistic condition due to presence of the bend at the top of the riser of the FIU-CFB and the long pipe running from the bend to the separation cyclone. For this reason, by considering the small distance between the most top pressure transducer and the exit of the riser, the best condition for the B.C. outlet was to use the values measured by the most top pressure transducer. In the next section, decency of this assignment will be demonstrated by the results that follow the experimental values in the top sections of the computation domain.

Table 5-3 shows the settings used for simulation of the $8^{\text {th }}$ case in Table 5-2. Parameter shown in this table which were not determined by experimentation, such $\mathrm{e}_{\mathrm{s}}$ and $\mathrm{e}_{\mathrm{w}}$, were obtained from available references on spent FCC in the literature (2012, Hong et al.). 
Table 5-3 Parameters for MFIX_TFM simulations of FIU-CFB case 8 (570-22)

\begin{tabular}{|l|l|l|l|}
\hline Property & symbol & value & unit \\
\hline Material & \multicolumn{3}{|c|}{ air and FCC } \\
\hline Particle diameter & $\mathrm{d}_{\mathrm{p}}$ & 79 & $\mu \mathrm{m}$ \\
\hline Particle density & $\mathrm{Ro}_{\mathrm{s}}$ & 845 & $\mathrm{~kg} / \mathrm{m}^{3}$ \\
\hline Air viscosity & $\mu_{\mathrm{g}}$ & $1.887 * 10^{-5}$ & $\mathrm{~Pa} . \mathrm{s}$ \\
\hline Superficial gas velocity & $\mathrm{U}_{\mathrm{g}}$ & 1.34 & $\mathrm{~m} / \mathrm{s}$ \\
\hline Solids mass flux & $\mathrm{G}_{\mathrm{s}}$ & 15.2 & $\left.\mathrm{~kg} / \mathrm{m}^{2} \mathrm{~s}\right)$ \\
\hline Solid inflow radial velocity & $\mathrm{V}_{\mathrm{x} \text {-solid }}$ & -0.231 & $\mathrm{~m} / \mathrm{s}$ \\
\hline Solid inflow axial velocity & $\mathrm{V}_{\mathrm{y} \text {-solid }}$ & -0.231 & $\mathrm{~m} / \mathrm{s}$ \\
\hline Single particle terminal velocity & $\mathrm{u}_{\mathrm{t}}$ & 0.077 & $\mathrm{~m} / \mathrm{s}$ \\
\hline Minimum fluidization voidage & $\varepsilon_{\mathrm{mf}}$ & 0.4 & - \\
\hline Packing limit & $\varepsilon_{\text {_s }} \mathrm{s}_{\max }$ & 0.63 & - \\
\hline Particle-particle coefficient restitution & $\mathrm{e}_{\mathrm{s}}$ & 0.9 & - \\
\hline Particle-wall coefficient restitution & $\mathrm{e}_{\mathrm{w}}$ & 0.99 & - \\
\hline Riser height & $\mathrm{h}$ & 3.8 & $\mathrm{~m}$ \\
\hline Riser diameter & $\mathrm{d}_{\text {riser }}$ & 0.0762 & $\mathrm{~m}$ \\
\hline Inlet port & $\mathrm{d}_{\text {in-2d }}$ & 0.055 & $\mathrm{~m}$ \\
\hline Outlet port & $\mathrm{d}_{\text {out-2d }}$ & 0.038 & $\mathrm{~m}$ \\
\hline Mesh size in traverse direction & IMAX & 30 & - \\
\hline Mesh size in axial direction & JMAX & 150 & - \\
\hline
\end{tabular}

The hybrid model introduced in chapter 3 of this dissertation and in the form of $F_{\text {drag.std }}$ $\rightleftharpoons \mathrm{F}_{\text {drag.clu. }}$ is used for modeling momentum exchange between gas and solid. For this purpose, the standard model is the Syam-O'Brien model and the switching operation is based on the cohesive index, Ha. The form of the Hybrid model is $g(\mathrm{Re}) *$ Syam-O’Brien, where $\mathrm{g}(\mathrm{Re})$ is the cluster-aware model introduced earlier in chapter 3 . The governing equations of the Naiver-Stokes and drag models are available in Chapter 3. Table shows the simulation set up for the cases observed in this simulation. 


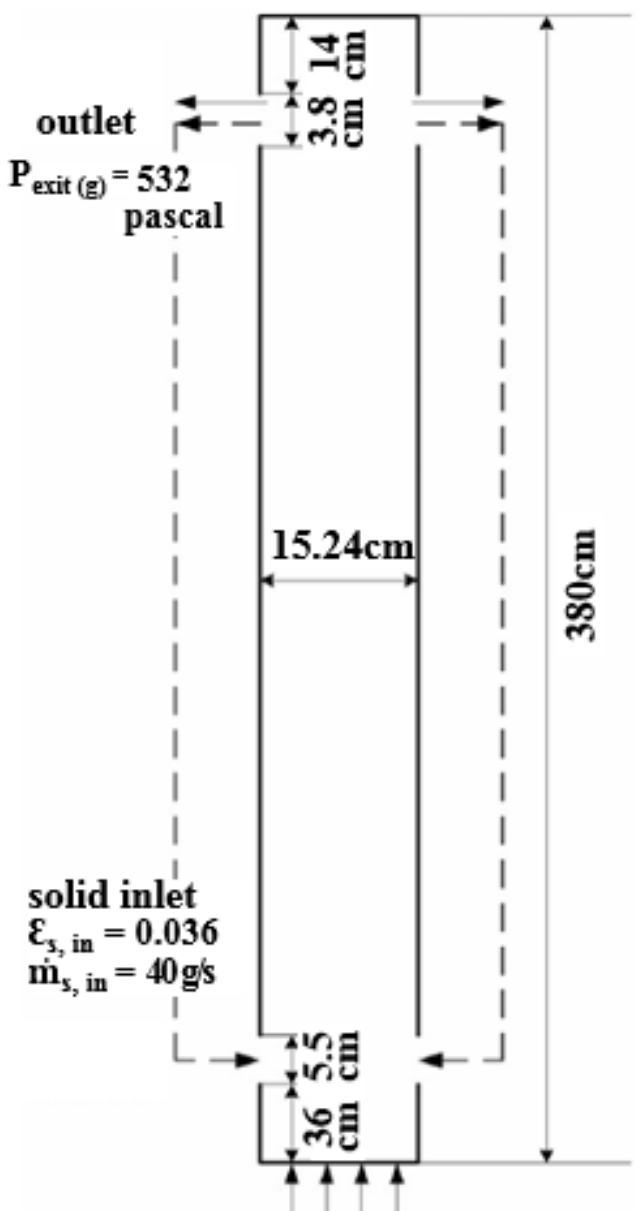

Gas inlet $\mathrm{U}_{\mathrm{g}}=1.34 \mathrm{~m} / \mathrm{s}$

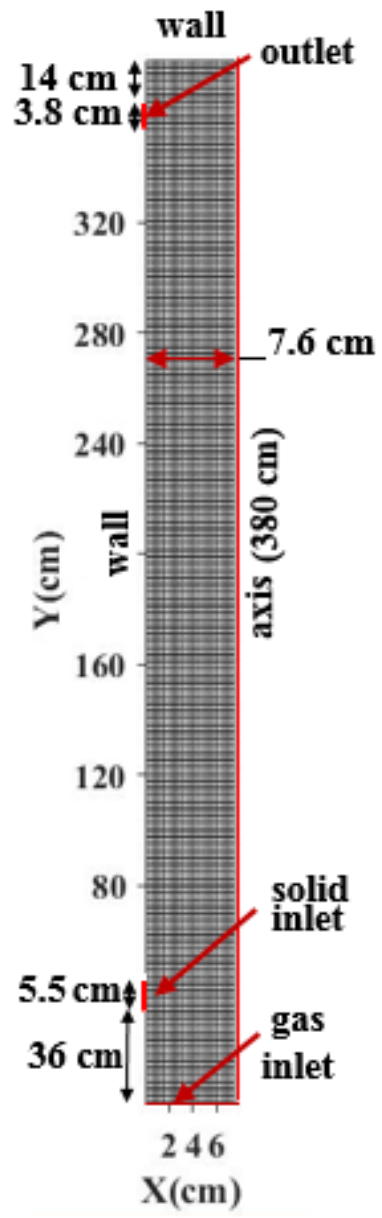

Figure 5-11 Computational domain representing the fluidization problem, (on left): schematic of the computational domain with boundary values, (on right): computational grid $(30 \times 150)$

\subsubsection{Results and Discussion}

Geometry and boundary conditions from the test case designated as case 570-22 in Table 5-2 were used to set up three TFM simulations in MFIX. The Syam-O’Brien along with and the AGDSM1 and AGDSM11 hybrid models were used to compute the momentum exchange coefficient between the gas and solid in these simulations. AGDSM1 and AGDSM11 hybrid models have the form of $F_{\text {drag. }}$ standard $\rightleftharpoons$ TGS and 
$\mathrm{F}_{\text {drag.standard }} \rightleftharpoons \mathrm{g}(\mathrm{Re})^{*} \mathrm{~F}_{\text {drag. standard, }}$ respectively and details of the models are available in chapters 2 and 4 of this dissertation.

The simulation results of the test case designated as case 570-22 in Table 5-2, are shown in Figure. The results are time averaged after mean quantities, e.g., pressure, gas velocity, and void fraction, reached steady values. Pressure profiles along the riser are shown for both the Syam-O'Brien and the hybrid model, named as AGDSM11. Figure 5-12 shows the contours of void fraction associated with the three drag models. The contours are very similar for all the models and correctly exhibit the inflow of solid into the fluidization column. In addition, complex flow structures like solid streamers and gas-solid mixing in the bottom section of the riser are observed in the computational domains of all three models. However, the difference between models could be better evaluated in the presence of the experimental measurements and visualizations of the flow. Visual information such as large filed images of the entire fluidization column or large sections of the unit, required more expensive tools and equipment such as X-ray imaging equipment which was not in the scope of the present study. For this purpose, we relied on available extracted data of measurement, such as the static pressure along the fluidization column of the FIU-CFB facility. 


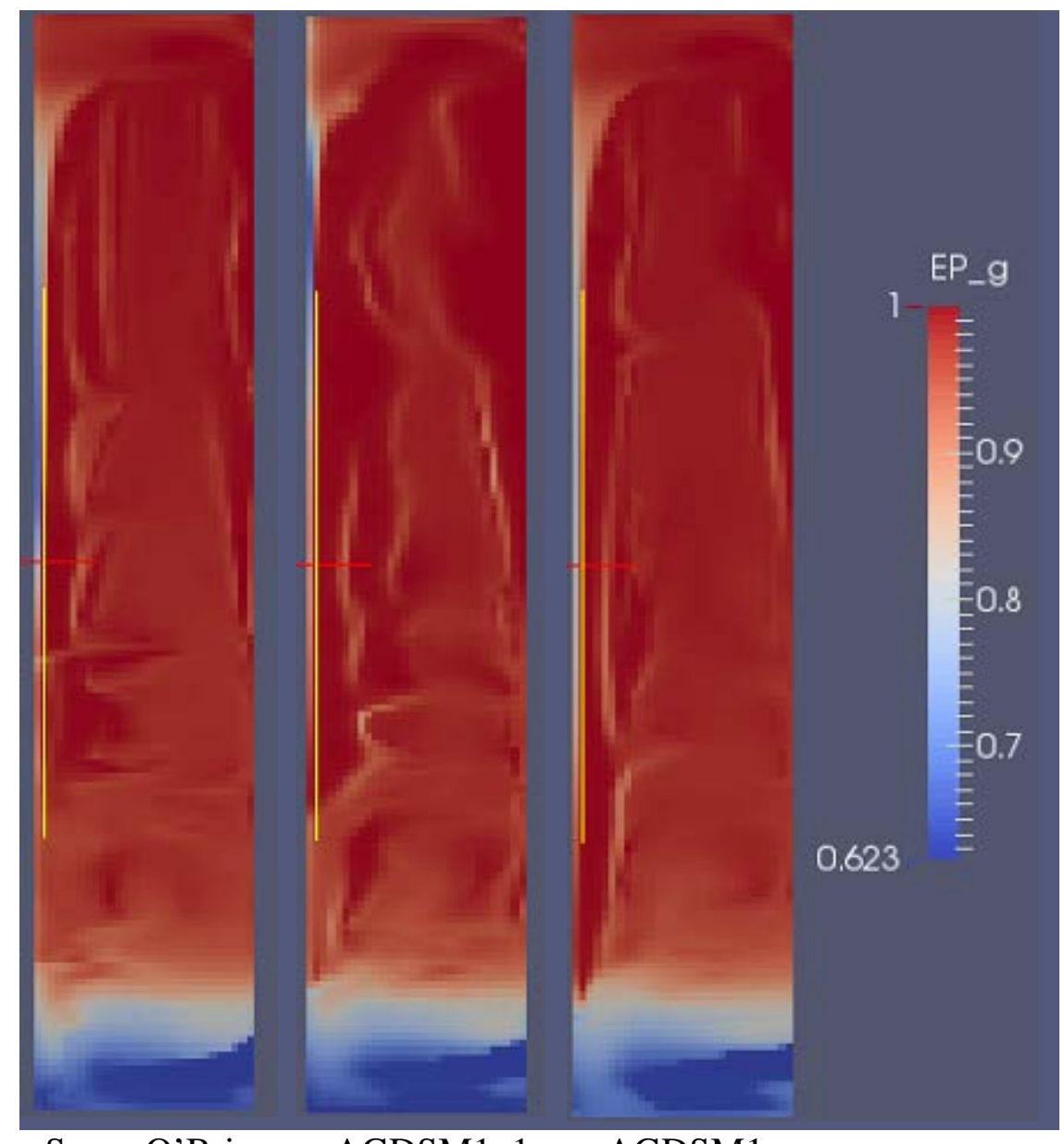

Syam-O’Brien AGDSM1_1 AGDSM1

Figure 5-12 Contour of void fraction $\left(\varepsilon_{\mathrm{g}}\right)$ from three TFM simulations of the fast fluidization flow inside FIU-CFB, drag models: Fdrag.std (Syam-O’Brien), AGDSM1 $\left(\mathrm{F}_{\text {drag. }}\right.$ std $\rightleftharpoons$ TGS $)$, and AGDSM11 ( $\mathrm{F}_{\text {drag. }}$ std $\left.\rightleftharpoons \mathrm{g}(\mathrm{Re}) * \mathrm{~F}_{\text {drag. }} \mathrm{std}\right)$

Figure 5-13 shows a comparison between the simulation results with the measured data along the fluidization column. This figure shows that simulation results are in decent agreement with the experimental measurements. However, the pressure models predicted higher pressure at the air inlet (bottom of the domain) and this data was required from the experiment for better investigation. This pressure could be obtained by subtracting the pressure drop across the distributor plate from the plenum pressure (the pressure measured below the distributor plate, $\mathrm{Pp}$ ). The value of the pressure drop across the 
distributor was 36psi (2482 Pascal) according to the table of pressure loss-flow rate published by the manufacturer of the plate $\left(\right.$ Mott $^{\circledR}$ Corporation) for the air flow of 87 acfm. Thus, the pressure value on the surface of the distributor plate was obtained as 1064 Pascal for the measured plenum pressure of 3546.1. However, all the models predicted a value of 2142.1 at this location.

The significant difference between measurement and the simulations at the air inlet port suggested that in numerical simulations with the indicted air velocity and solid mass inflow rate, higher amount of total pressure was needed at the gas inlet to overcome the weight of the solid material and to fluidize it. This discrepancy could be caused by small uncertainties in measurement of solid particle size, density, and solid mass rate into the system. In addition, part of the discrepancies might be rooted in the assumption of perfect similarity between the $2 \mathrm{~d}$ axisymmetric simulation and the real $3 \mathrm{~d}$ existence of the test domain. However, models followed the experiment in regards to the rate of pressure decrease along the riser of the fluidization bed and more attention is paid to the comparison of models above the location of the first pressure transducer. 
Time-Area Averaged Pressure profile

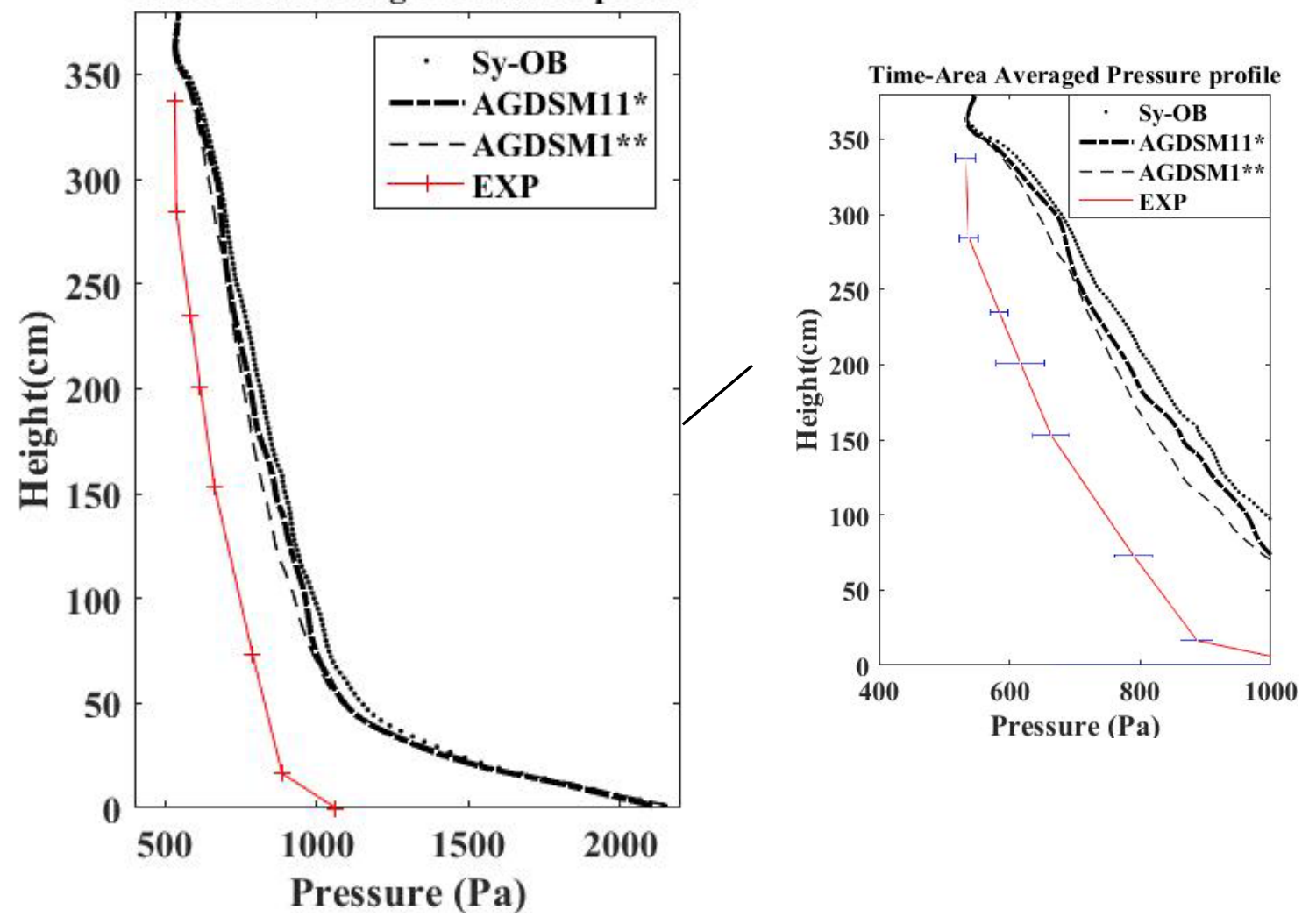

Figure 5-13 comparison of pressure profiles along the fluidization column of FIU-CFB * Ha-THS $=1 \times 10^{-10} * *$ Ha-THS $=1 \times 10^{-10}$

Furthermore, results indicated that for $16.3 \mathrm{~cm} \leq$ height $\leq 350$, lower pressure values were obtained by the hybrid models in comparison with the standard model. The best agreement between the simulation and experiment was obtained for the AGDSM1 model. The second best agreement was obtained by the AGDSM11 model these ratings agree with comparison of performance improvements tabulated in Table 3.1 for the entire domain. As a trivial fact, presence of lower pressure drop along the column of the fluidized bed is indicative of lower resistance force to the air flow. This resistance is mainly due to the drag forces between the gas and solid phases, which are significantly 
larger than frictional losses caused by the riser walls. In Figure 5-13, inverse of slope of profiles represent the pressure drop between any two arbitrary points. Therefore, by considering the profiles in Figure 5-13, it is easy to associate the smallest and largest pressure drops to the AGDSM1 and Syam-O'Brien models, respectively. Thus, it is rational to conclude that the occurrence of the best performance is associated with the smallest computed drag force. Conversely, overestimation of the drag force degrades the accuracy of the numerical modeling, which supports the findings of McKeen and Pugsley (2003), Syamlal and O'Brien (2003), and Zimmermann and Taghipour (2005).

\subsection{Conclusion}

Experimental and numerical study of fast fluidization flow was conducted and presented in this chapter of the dissertation. A series of gas-solid fast fluidization flow experiments with different air and solid flow rate s were successfully conducted inside the FIU-CFB facility. In the experiments, increase of the pressure due to the increase of solid circulation rate and presence of particle clusters in the flow were confirmed. Measurements of different quantities such as density and particle size for the FCC powder, volumetric flow rate of air, mass flow rate of the circulated solid, and pressure along the column of the fluidization column were conducted.

Numerical investigation of an experimental case was conducted in a two-dimensional axisymmetric domain by conserving the mass, momentum and energy balances between the two-dimensional and the real three-dimensional systems. Based on the close agreement between the numerical and simulation results for all three models, it is 
concluded that numerical modeling was successful in capturing the main features of the flow under investigation. In addition, better performance was observed by the clusteraware hybrid models by predicting lower pressure and lower pressure drop values along the riser in comparison to the standard model. Overestimation of the drag force in the numerical simulation resulted in more deviation of the results from the experimental values. Furthermore, the rating of the hybrid models in regards to accuracy improvement was consistent to the ratings presented in the chapter 3 of this dissertation and in the work of Abbasi et al. (2015). We can conclude that more reduction of the drag forces by the hybrid models contributed to more improvements of the agreement between the simulations and the experiment.

\subsection{References}

Abbasi Baharanchi A., Gokaltun S., Dulikravich G.S., 2015, Performance Improvement of Existing Drag Models in Two-Fluid Modeling of Gas-Solid Flows Using a PR-DNS Based Drag Model, Journal of Powder Technology, Volume 220, pp 63-69

Agrawal K., Loezos P.N., Syamlal M., Sundaresan S., 2001, The role of meso-scale structures in rapid gas solid flows, Journal of Fluid Mech. (2001), vol. 445, pp. 151-185.

Brereton C.M., Grace J.R., 1993, End Effects in CFB Hydrodynamics, Circulating Fluidized Bed Technology, No.4, pp 137-144

Cabezas-Go'mez L., Ce'sar da Silva R, Navarro H.A, Milioli F.E., 2008, Cluster identification and characterization in the riser of a circulating fluidized bed from numerical simulation results, Journal of Applied Mathematical Modelling, Volume 32, 327-340

Cocco R. Shaffer F., Hays R.S.B., Karri R., Knowlton T., 2010, Particle clusters in and above fluidized beds, Journal of Powder Technology, Volume 203, pp 3-11

Cocco R., Shaffer F., Karri S.B.R., Hays R., Knowlton T., 2011, Particle Clusters In Fluidized Beds, ECI Conference on The 13th International Conference on Fluidization New Paradigm in Fluidization Engineering 
Cocco R. Shaffer F., Hays R.S.B., Karri R., Knowlton T., Introduction to fluidization , 2014, CEP Magazine AIChE

Gidaspow D., Tsuo Y.P., Luo K.M., 1989, Computed and experimental cluster formation and velocity profiles in circulating fluidised beds, Fluidisation VI, International Conference on Fluidisation, Banff, Canada, pp. 81-88.

Harris A.T., Davidson J.F., Thorpe R.B., The prediction of particle cluster properties in the near wall region of a vertical riser (200157), Journal of Powder Technology Volume 127, pp 128- 143

Igci Y., Sundaresan S. ,2011, Constitutive Models for Filtered Two-Fluid Models of Fluidized Gas_Particle Flows, Industrial Engineering Chemistry Research, Issue 50, pp 13190-13201

Li J., Ge W., Wang W., Yang N., Liu X., Wang L., He X., Wang X., Wang J., Kwauk M., 2013, From Multiscale Modeling to Meso-Science, A Chemical Engineering Perspective Principles, Modeling, Simulation, and Application, Springer Heidelberg New York Dordrecht London, Library of Congress Control Number: 2012954048, DOI 10.1007/978-3-642-35189-1

Lim k.S., Zhou J, Finlay C., Grace J.R., Lim C.J., Brereton C.M.H., 1995, Cluster descending velocity at the wall of circulating fluidised bed risers, in: M. Kwauk, J. Li (Eds.), Circulating Fluidised Bed Technology, pp. 218-223.

Lu B., Wang W., Li J., 2009, Searching for a mesh-independent sub-grid model for CFD simulation of gas-solid riser flows, Journal of Chemical Engineering Science, Volume 64, Issue 15, pp 3437-3447

McKeen T., Pugsley T., 2003, Simulation and Experimental Validation of a Freely Bubbling Bed of FCC Catalyst, Journal of Powder Technology, Volume 129, Issues 1-3, pp 139-152

Milioli, C. C., Milioli F. E., Holloway W., Agrawal, K., Sundaresan S., 2013, Filtered Two-Fluid Models of Fluidized Gas-Particle Flows: New Constitutive Relations, AIChE Journal, Volume 59, Issues 9, pp 3265-3275,

Qi H.Y., Li F., Xi B, You C., 2007. Modeling of Drag with the Eulerian Approach and EMMS Theory for Heterogeneous Dense Gas-Solid Two-Phase Flow, Journal of Chemical Engineering Science, Volume 62, Issue 6, pp 1670-1681

Rhodes M.J., Mineo H., Hirama T., 1992, Particle motion at the wall of a circulating fluidized bed, Powder Technol. 70, 207-214. 
Sharma N., Patankar N., 2005, A fast computation technique for the direct numerical simulation of rigid particulate flows, Journal of Computational Physics, Volume 205, Issue 2, pp 439-457

Soong C.H., Tuzla K., Matsen J., Chen J.C., 1993, Identification of particle clusters in circulating fluidized beds, in: Avidan (Ed.), Circulating Fluidized Bed Technology IV Engineering Foundation, New York, p. 615,

van Deemter and van der Laan, 1961, Momentum and Energy Balances For Dispersed Two-Phase Flow, Journal of Applied Science Research, section A, Vol. 10, pp 102-110

Wang J., Ge W., Li J., 2008, Eulerian Simulation Of Heterogeneous Gas-Solid Flows in CFB Risers: EMMS-based Sub-Grid Scale Model with a Revised Cluster Description, Journal of Chemical Engineering Science, Volume 63, Issue 6, pp 1553-1571

Wang J., 2009, A Review of Eulerian Simulation of Geldart A Particles in Gas-Fluidized Beds, Journal of Industrial and Engineering Chemistry Research, volume 48, Issue 12, pp 5567-5577

Wang W., Li J., 2007, Simulation of Gas-solid Two-phase Flow by a Multiscale CFD Approach: Extension of the EMMS Model to the Sub-grid Scale Level, Journal of Chemical Engineering Science, Volume 62, Issues 1-2, pp 208-231.

Xu J., Zhu J.X., 2011, Visualization of particle aggregation and effects of particle properties on cluster characteristics in a CFB riser, Chemical Engineering Journal, Volume 168, Issue 1, pp 376-389

Ye M., van der Hoef M.A., Kuipers J.A.M., 2005a, From Discrete Particle Model To A Continuous Model of Geldart A Particles, Journal of Chemical Engineering Research and Design, Volume 83, Issue 7, pp 833-843

Ye M.A, van der Hoef M.A., Kuipers J.A.M., 2005b, The Effects of Particle and Gas Properties on the fluidization, Journal of Chemical Engineering Science, Volume 60, Issue 16, pp 4567- 4580

Ye M. A., van der Hoef M.A., Kuipers J.A.M, 2008, Two-fluid modeling of Geldart A particles in gas-fluidized beds, Journal of Particuology, Volume 6, Issue 6, pp 540-548

Yang N., Wang W., Ge W., Li J., 2003, CFD Simulation of Concurrent-up Gas-Solid Flow in Circulating Fluidized Beds with Structure-Dependent Drag Coefficient, Journal of Chemical Engineering, Volume 96, Issues 1-3, pp 71-80 
Yang N., Wang W., Ge W., Li J., 2004, Simulation of Heterogeneous Structure in a Circulating Fluidized-Bed Riser by Combining the Two-Fluid Model with the EMMS Approach

Yang T-Y, Lue L-P, Multiresolution Analysis on Identification and Dynamics of Clusters in a Circulating Fluidized Bed, American Institute of Chemical Engineers AIChE J, Volume 55: 612-629, 2009

Yerushalmi J., Gluckman M.J., Graff R.A., Dobner S., Squires A.M,, 1975, Production Of Gaseous Fuels From Coal In The Fast Fluidized Bed, Fluidization Technology, Volume 11

Zhang D.Z., Vanderheyden W.B., 2002, The Effects of Mesoscopic Structures on the Macroscopic Momentum Equations for Two-Phase Flows. International Journal of Multiphase Flow, Volume 28, Issue 5, pp 805-822 


\section{CHAPTER 6: SUMMARY AND CONCLUSION}

Direct inclusion of cluster prediction mechanism based on the cohesive inter particle forces into the drag models in the Two-Fluid Modeling approach is complex and one of the least studied subjects in the literature. In this work, a numerical model was developed in the MFIX-TFM to predict particle clustering based on the nature of the competing attractive and repulsive interparticle interactions for the particles in motion. This model was used in two cluster-aware hybrid models developed in the MFIX-TFM for simulation of two fluidization flows, involving FCC as a powder in Geldart A regime with strong interparticle cohesive forces. Model validation in this work was performed using available experimental data from the literature and the data measured for a fast fluidization flow of air-FCC inside the riser of FIU-CFB facility.

First hybrid model with the ability of switching from a standard drag model to a PRDNS-based drag model was developed and incorporated into the MFIX-TFM code. Three versions of the first hybrid model were generated by using three standard drag models. Benchmark validation of the models against data from an experiment in the literature indicated that versions of the hybrid models were significantly in closer agreement with the experimental data rather than all three standard models. It was indicated that generally smaller values of the threshold in the cluster prediction model resulted in better accuracy of the models, since the smallest chances of clustering were considered for migration from the standard model to the PR-DNS model. 
Analysis of the results indicated that on average the PR-DNS model predicted the smallest drag forces in comparison to the three standard models. Results indicated that switching to the PR-DNS model was a stable mechanism that encouraged less switch operation by reducing the cohesive index between particles. Spatially averaged results indicated that all three hybrid models predicted normalized drag forces less than one. The largest improvement, in terms of relative error, was obtained for the standard model that was most different from its associate hybrid model in terms of normalized drag force.

Later, the second hybrid model with the ability of switching from a standard drag model to a cluster-aware PR-DNS from the literature was developed in the MFIX-TFM. This model was used in simulation of a fluidization experiment in the literature and its results were compared to both the experimental data and the simulation results of the first hybrid model, for benchmark validation and verification of the model. Results indicated that this hybrid cluster-aware model was significantly in closer agreement with the experimental data in the dense regions of the computational domain in comparison to both the standard and the fist hybrid drag models. Inclusion of the smallest chances of particle clustering (via reduction of the threshold criterion in the cluster prediction mechanism) increased the accuracy of the simulation results significantly in the dense regions of the computational domain. Nevertheless, this led to significant degradation of the accuracy of simulation results in the dilute regions of the computational domain. Therefore, by considering the range of validity of the cluster-aware PR-DNS model used in this dissertation, i.e., $\Phi=0.087$, a wider range of validity of the cluster-aware PR-DNS, 
e.g., $\Phi \sim 0.0087$ to 0.3 , might contribute to better predictions in the entire computational domain.

Finally, an experiment was designed and developed for the purposes of better understanding the fast fluidization flow of cohesive FCC particles, visualization of clusters, and extraction of data for benchmark validations. Two dimensional axisymmetric simulations with three drag model, i.e., a standard model, and the first and second cluster-aware hybrid models, were considered for benchmark validations against the measurement data.

Experimental results demonstrated the presence of clusters of the FCC particle of different shapes and sizes. Pressure data and boundary conditions were successfully measured at different air and solid flow rates and results exhibited an increase of pressure along the riser of the test facility with the increase of solid loading at constant air flow rate. Simulation results of the three models indicated acceptable agreement with the experimental data, except in the regions close to the air inlet boundary, where higher pressure was predicted by simulations. This discrepancy might be improved by considering the uncertainties in measurements of boundary conditions, material characterization. However, Numerical results indicated better performance of the clusteraware hybrid models by predicting lower pressure and lower pressure drop values along the riser in comparison to the standard model. It is concluded that overestimation of the drag force is the issue with the standard models when it comes to the accuracy of simulation results. 
The findings from this work provide insight of how performance of the TFM approach, which is suitable for simulation of fluidization flows in industrial scales, can be improved by utilization of cluster-aware hybrid models. In fact, it was shown in this dissertation that by using the information provided by particle resolved direct numerical simulations around particle clusters, significant modifications could be obtained for industrial scale flow simulations. However, there is more room for more improvement. Replacement of the standard models used in this dissertation with the TGS, as a particle resolved model, can construct a more elaborate hybrid model in the form of TGS $\rightleftharpoons \mathrm{g}(\mathrm{Re}, \Phi, \mathrm{Ha}) * \mathrm{TGS}$ and this requires full identification of the g function for wide ranges of Reynolds number, solid volume fraction, and cohesion index. Moreover, the switching mechanism can be omitted by directly including the Ha in the definition of a blending function in the form of $\Gamma(\mathrm{H})$, which creates a drag model in the form of $\mathrm{F}_{\text {drag }}=\Gamma(\mathrm{H})^{*} \mathrm{TGS}+(1-\Gamma(\mathrm{H}))^{*}$ $\mathrm{g}(\mathrm{Re}, \Phi, \mathrm{Ha}) *$ TGS. 
VITA

\section{AHMADREAZ ABBASI BAHARANCHI}

1985

2014

2010

2007

2012-present

2011-2012
Born, Isfahan, Iran

PhD candidate, Mechanical Engineering, Department of mechanical and Material Engineering Florida International University, Florida, USA

MSc Mechanical Engineering, Faculty of Mechanical Engineering, , Technological University of Malaysia, Johor Bahru, Malaysia

BSc, Mechanical Engineering, Isfahan University of Technology, Isfahan, Iran

Research Assistant, Applied Research Center, Florida, International University, Florida, USA

Teaching Assistant, Department of mechanical and Material Engineering Florida International University, Florida, USA

\section{PUBLICATIONS AND PRESENTATIONS}

Ahmadreza Abbasi Baharanchi, Maximiliano Edrei, Seckin Gokaltun, Dwayne McDaniel, A Dissipation-Based Method for Improving the Accuracy of Computational Fluid Dynamics Simulations of High Level Non-Newtonian Waste, Journal of NonNewtonian Fluid Mechanics , 2016 (under review)

Abbasi Baharanchi A., Gokaltun S., Dulikravich G.S., Investigation Of Parameters Contributing To Improvements Of Two Fluid Modeling Of A Fludization Flow, Journal of Powder Technology, 2016 (under review)

Abbasi Baharanchi A., Gokaltun S., Dulikravich G.S., A Cluster-Aware Drag Model For Simulation Of Particle-Gas Flows Involving Cohesive Interparticle Forces, Journal of Powder Technology, 2016 (under review)

Abbasi Baharanchi A., Gokaltun S., Dulikravich G.S., Simulation Of Fast Fluidization Flow Inside The FIU_CFB Facility Using Enhanced Cluster-Aware Drag Models In MFIX-TFM, Journal of Powder Technology, (under review)

2015 Abbasi Baharanchi A., Gokaltun S., Dulikravich G.S., Abbasi Baharanchi 
A., Gokaltun S., Dulikravich G.S., 2015, Performance Improvement of Existing Drag Models in Two-Fluid Modeling of Gas-Solid Flows Using a PR-DNS Based Drag Model, Journal of Powder Technology, Volume 220, pp 63-69

2013 American Society of Mechanical Engineers, Numerical Approach For The Simulation Of Internal Nozzle Flow In A Pressure Swirl Atomizer Using Different Turbulent Models And Towards An Effective Inlet WeberNumber, Abbasi A.A. Baharanchi, A.N. Darus, S.Gokaltun , S.Eshraghi , ASME 2013 International Mechanical Engineering Congress \& Exhibition , IMECE13, November 12-15, 2013, San Diego, CA, USA

2013 Workshop on Multiphase Flow Science, Ahmadreza Abbasi Baharanchi, Seckin Gokaltun, Norman Manrue, George S. Dulikravich, An Experimental Study Using High Speed Imaging Of Clustered Particles For A More Accurate Drag Model In MFIX, August 6-7, 2013, Morgantown, WV, USA

2012 American Society of Mechanical Engineers, Introduction of a Threshold Weber Number For Inclusion Of Surface Tension Force In Simulation Of Nozzle Internal Flow In Pressure Swirl Atomizers, A.A. Abbasi Baharanchi, A.N.Darus, M.Ansari, ASME 2012 International Mechanical Engineering Congress and Exposition, IMECE12, November 9-15, 2012, Huston, Texas, USA, IMECE2012-87128

2012 American Society of Mechanical Engineers, Numerical simulation of laminar forced convection heat transfer of two square prisms inside nanofluids, S. M. H. Jayhooni, M. H. Nowzari, K. Jafarpur, A.A. Abbasi Baharanchi, ASME 2012 International Mechanical Engineering Congress and Exposition, IMECE2012-89962, November 9-15, 2012, Huston, Texas, USA

2012 American Society of Mechanical Engineers, Effect of Staggered Configurations on Laminar Forced Convection Heat Transfer From Square Cylinders Inside Water/CuO Nanofluid, S. M. H. Jayhooni, M. H. Nowzari, K. Jafarpur, A.A. Abbasi Baharanchi, ASME 2012 International Mechanical Engineering Congress and Exposition, IMECE2012-89958, November 9-15, 2012, Huston, Texas, USA 